ACCEPTED MANUSCRIPT

\title{
Boundary and defect CFT: Open problems and applications
}

To cite this article before publication: Natan Andrei et al 2020 J. Phys. A: Math. Theor. in press https://doi.org/10.1088/1751-8121/abb0fe

\section{Manuscript version: Accepted Manuscript}

Accepted Manuscript is "the version of the article accepted for publication including all changes made as a result of the peer review process, and which may also include the addition to the article by IOP Publishing of a header, an article ID, a cover sheet and/or an 'Accepted

Manuscript' watermark, but excluding any other editing, typesetting or other changes made by IOP Publishing and/or its licensors"

This Accepted Manuscript is @ 2020 IOP Publishing Ltd.

During the embargo period (the 12 month period from the publication of the Version of Record of this article), the Accepted Manuscript is fully protected by copyright and cannot be reused or reposted elsewhere.

As the Version of Record of this article is going to be / has been published on a subscription basis, this Accepted Manuscript is available for reuse under a CC BY-NC-ND 3.0 licence after the 12 month embargo period.

After the embargo period, everyone is permitted to use copy and redistribute this article for non-commercial purposes only, provided that they adhere to all the terms of the licence https://creativecommons.org/licences/by-nc-nd/3.0

Although reasonable endeavours have been taken to obtain all necessary permissions from third parties to include their copyrighted content within this article, their full citation and copyright line may not be present in this Accepted Manuscript version. Before using any content from this article, please refer to the Version of Record on IOPscience once published for full citation and copyright details, as permissions will likely be required. All third party content is fully copyright protected, unless specifically stated otherwise in the figure caption in the Version of Record.

View the article online for updates and enhancements. 
Subject Areas:

Theoretical Physics, Mathematical Physics

\section{Keywords:}

Conformal Field Theory, Boundaries and Defects, Non-Perturbative Effects, Holographic Duality, Supersymmetry

\section{Author for correspondence:}

A. O'Bannon

e-mail: a.obannon@soton.ac.uk
Boundary and Defect CFT: Open Problems and Applications

N. Andrei ${ }^{1}$, A. Bissi ${ }^{2}$, M. Buican

J. Cardy ${ }^{4,5}$, P. Dorey ${ }^{6}$, N. Drukker ${ }^{7}$

J. Erdmenger ${ }^{8}$, D. Friedan ${ }^{1,}$ D. Fuirsaev ${ }^{10}$,

A. Konechny ${ }^{11,12}$, C. Kristjansen ${ }^{13}$

I. Makabe ${ }^{7}$, Y. Nakayama ${ }^{14}$, A. O'Bannon ${ }^{15}$,

R. Parini ${ }^{16}$, B. Robinson ${ }^{15}$, S. Ryu ${ }^{17}$,

C. Schmidt-Colinet ${ }^{18}$, V. Schomerus ${ }^{19}$,

C. Schweigert ${ }^{20}$, and G.M.T. Watts ${ }^{7}$

${ }^{1}$ Dept. of Phys., Rutgers Univ., Piscataway, NJ, USA.

${ }^{2}$ Dept. of Phys. and Astro., Uppsala Univ., SE

${ }^{3}$ CRST and SPA, Queen Mary Univ. of London, UK

${ }^{4}$ Dept. of Phys., Univ. of California, Berkeley, CA, USA

${ }^{5}$ All Souls College, Oxford, UK

${ }^{6}$ Dept. of Math. Sci., Durham Univ., UK

${ }^{7}$ Dept. of Maths, King's College London, UK

${ }^{8}$ Julius-Maximilians-Univ. Würzburg, DE

${ }^{9}$ Natural Science Inst., Univ. of Iceland, IS

${ }^{10}$ Dubna State Univ., Dubna, RU

${ }^{11}$ Dept. of Maths, Heriot-Watt Univ., Edinburgh, UK

${ }^{12}$ Maxwell Inst. for Math. Sci., Edinburgh, UK

${ }^{13}$ Niels Bohr Inst., Copenhagen Univ., , DK

${ }^{14}$ Dept. of Phys., Rikkyo Univ., Tokyo, JP

${ }^{15}$ STAG Research Centre, Univ. of Southampton, UK

${ }^{16}$ Department of Mathematics, University of York, UK

${ }^{17} \mathrm{~J}$. Franck Inst. \& KCTP., Univ. of Chicago, IL, USA

${ }^{18}$ Arnold Sommerfeld Center, Univ. München, DE

${ }^{19}$ DESY Theory Group, DESY Hamburg, Hamburg, DE

${ }^{20}$ Fachbereich Math., Univ. Hamburg, Hamburg, DE

A review of Boundary and Defect Conformal Field Theory: Open Problems and Applications, following a workshop held at Chicheley Hall, Buckinghamshire, UK, 7-8 Sept. 2017. We attempt to provide a broad, bird's-eye view of the latest progress in boundary and defect conformal field theory in various sub-fields of theoretical physics, including the renormalization group, integrability, conformal bootstrap, topological field theory, supersymmetry, holographic duality, and more. We also discuss open questions and promising research directions in each of these sub-fields, and combinations thereof. 


\section{Introduction and Overview ${ }^{1}$}

This review is based on results presented at the workshop "Boundary and Defect Conformal Field Theory: Open Problems and Applications" held at Chicheley Hall in Buckinghamshire, UK on 7 and 8 September 2017. Each section that follows was authored by the speaker listed in the footnote attached to the corresponding section title. In this introduction we provide context and motivation for this review, summarize the contents of each section, and extract a few general lessons from what follows. We therefore hope to provide a bird's-eye view of the different directions that research into conformal field theories (CFTs) with boundaries or defects is taking.

Quantum field theory (QFT) lies at the heart of much of modern theoretical physics: it describes systems in particle physics, condensed matter physics, and even quantum gravity, via holographic duality. Combined with the machinery of the renormalization group (RG), one can in principle systematically study an enormous variety of phenomena at different length scales in QFT-and therefore in nature.

CFTs occupy privileged places in the space of QFTs-they lie at the endpoints of RG flows. Therefore, they often characterize the ultraviolet (UV) and infrared (IR) limits of QFTs. Moreover, CFTs describe critical phenomena and the worldsheet theory in string theory. They also provide important testing grounds for integrability, duality, and other general phenomena in QFT.

CFTs are powerful because they are highly symmetric. In particular, CFTs are scale-invariant by definition, and so all correlation lengths are infinite. CFTs are also invariant under translations, rotations, boosts, and inversions. These symmetries constrain correlation functions, sometimes completely, providing a powerful non-perturbative approach to many aspects of QFT.

However, no real-world system has infinite size-boundary effects are always important. Moreover, all real-world systems involve impurities, differently-ordered regions separated by domain walls, and other types of defects that break translational and rotational symmetries to subgroups ${ }^{2}$. In short, the application of CFT to the real world necessarily entails studying boundary CFTs (bCFTs) and defect CFTs (dCFTs).

The reduced symmetry of bCFTs and dCFTs compared to CFTs loosens spectral constraints, making correlation functions richer and more intricate. For example, in a bCFT, scalar operators can have non-zero one-point functions, which are generally forbidden in a CFT. However, bCFTs and dCFTs often retain enough symmetry to provide calculable non-perturbative information about many systems. For example, bCFT provides a solution to the single-impurity Kondo problem [1] and a fully non-perturbative definition of D-branes and other spacetime defects in string theory [2].

Remarkably, bCFT and dCFT can also provide insight into RG flows beyond the critical endpoints. Indeed, consider a CFT with a relevant deformation that triggers an RG flow to another CFT. Now, imagine integrating that relevant deformation over half the spacetime. Then, in the IR, the result is an interface between the UV and IR CFTs, called an RG interface or RG domain wall [11]. Such a construction, though clearly sacrificing some of the spacetime symmetry, is potentially very powerful: the problem of classifying RG flows between CFTs maps onto the problem of classifying defects between CFTs-which should be simpler, because much of the machinery of CFT can be brought to bear. Similarly, if the relevant deformation produces a mass gap, then the resulting IR will be the UV CFT with a boundary, hence the problem of classifying gapped vacua maps to the simpler problem of classifying conformal boundary conditions. In

${ }^{1}$ This section was authored by Matthew Buican, from CRST and the School of Physics and Astronomy, Queen Mary University of London, London E1 4NS, UK, and Andy O'Bannon and Brandon Robinson from the STAG Research Centre, Physics and Astronomy, University of Southampton, University Road, Highfield, Southampton SO17 1BJ, UK. M.B. and A.O'B. are partially supported by Royal Society University Research Fellowships. M.B.'s work was supported by the Royal Society under the grant, "New Constraints and Phenomena in Quantum Field Theory." A.O'B. and B.R. were also supported by STFC consolidated grant ST/L000296/1.

${ }^{2}$ In general, a co-dimension one defect between CFTs (i.e. a domain wall) can be mapped to a boundary of the product CFT via the folding trick, as discussed for example in $\S 9$.

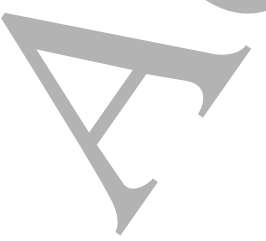


short, defects and boundaries allow CFT techniques to be extended to a much larger domain in the space of QFTs.

Given the incredible breadth of bCFT and dCFT, this review's goal was to bring together a diverse group of leading physicists working on different topics in these fields and search for common themes and unifying ideas. This review was also motivated by the desire to understand how recent developments that have reshaped CFT-like the modern conformal bootstrap, nonperturbative results in supersymmetry (SUSY), and recent advances in holography-combined with recent developments that have reshaped condensed matter physics-like topological phases and new results in integrability-have impacted bCFT and dCFT. Conversely, we wanted to know what impact bCFT and dCFT might have on these fields, i.e.what new applications of bCFT and dCFT may be possible.

To help orient the reader interested in the various different themes of this review, we have organized the contributions thematically. In the next three subsections, we identify these themes and point to a few ideas unifying the contributions within each theme.

\section{RG flows: Interfaces and boundaries}

The first six sections ( $\$ 2-\S 8$ ) address the behavior of boundaries and/or defects under RG flows. Of these, the first four ( $(2-\S 5)$ study boundaries and defects as maps between UV and IR CFTs, as described above. In particular, in $\S 2$ John Cardy describes a variational approach, in $\S 3$ Anatoly Konechny uses a combination of analytics and the truncated conformal space approach, in $\S 4$ Cornelius Schmidt-Colinet employs holography, and in § 5 Shinsei Ryu uses ideas from topology (and explores setups in which CFTs are themselves boundaries of higher-dimensional massive theories) ${ }^{3}$. The final two sections of the group each have a slightly different focus. In $\S 6$ Natan Andrei studies a purely boundary RG flow and describes the behavior of certain observables involving quantum dots coupled to Luttinger liquids, using techniques from integrability. In $\S 7$ Johanna Erdmenger explores impurity entropy in a holographic version of the Kondo model, involving an RG flow on an impurity coupled to a strongly-interacting CFT at large $N$. In $\S 8$ Patrick Dorey discusses boundary conditions that break integrability in a massive theory.

\section{Supersymmetric boundaries and defects}

The next four sections $(\S 9-\S 12)$ discuss aspects of SUSY bCFTs and dCFTs. In $\S 9$ Gerard Watts finds new non-topological defects in the tri-critical Ising model. In $§ 10$ Nadav Drukker discusses the SUSY multiplet for the displacement/operator of co-dimension one defects in $4 \mathrm{D} \mathcal{N}=1$ theories (the key role of the displacement operator has also been emphasized recently in $[3,4]$ ). In $\S 11$ Charlotte Kristjansen discusses holographic constructions of defects that interpolate between two copies of $4 \mathrm{D} \mathcal{N}=4$ super Yang-Mills theory with different rank gauge groups. In $\S 12$ Agnese Bissi explains techniques for computing loop corrections in AdS/CFT, which could be applied to study quantum effects in SUSY holographic dCFTs at subleading order in the $1 / N$ expansion.

\section{A toolbox for bCFTs, dCFTs, and beyond}

The final five sections ( $\S 13-\S 17)$ deal with creating new tools to analyze bCFTs, dCFTs, and their generalizations. In $\S 13$ Dmitri Fursaev discusses integrated conformal anomalies of bCFTs and dCFTs, and their use in determining various observables. In § 14, Volker Schomerus develops new tools to analyze correlation functions of defects via connections to CalogeroSutherland models (which should allow the application of conformal bootstrap techniques to these correlators). In $\S 15 \mathrm{Yu}$ Nakayama implements the conformal bootstrap in what turns out to be one of the simplest curved backgrounds: real projective space (states in this space are closely related to boundary states in flat space CFT). In $\S 16$ Christoph Schweigert develops the

${ }^{3}$ Note that when the IR phase is massive but contains non-trivial topological degrees of freedom, one still sometimes refers, as in $\S 5$, to the object separating the UV and IR as a defect or domain wall.

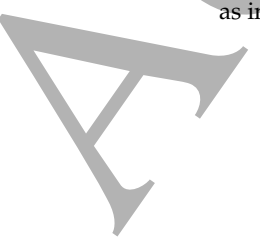


proper three-dimensional topological field theory framework in which to analyze rational CFTs and, for logarithmic CFTs, a modular functor constructed by a "Lego-Teichmüller game" (which ultimately leads to a better understanding of boundary conditions in these theories [189]). Finally, in § 17 Daniel Friedan explores entirely new species of quantum theories built from defects.

\section{Open Problems and Future Directions}

This review presents a very diverse set of results in bCFT, dCFT, and beyond, and suggest various interesting open questions. Here we briefly mention just a few. Are there general principles that select the RG domain wall(s)/boundary state(s) from the possible set connecting the UV and IR endpoints of RG flows? What more can SUSY tell us about bCFTs and dCFTs, for example via SUSY localization? Are there boundary or defect " $c$-theorems" generalizing the $g$ theorem $[208,209]$ of two-dimensional bCFT to higher dimensions? To what extent can SUSY, the conformal bootstrap, integrability, topological field theory, and the other techniques discussed in these proceedings be used to address such questions? Could they also lead to the discovery of new conformal boundary conditions, defects, or even entirely new perspectives on what QFT is?

We hope that this review will inspire new research into boundaries and defects in CFT, by making connections between sub-fields, finding novel applications, and advancing the state of the art of bCFT and dCFT, all building upon the remarkable progress summarized below.

\section{Bulk Renormalization Group Flows and Boundary States ${ }^{4}$}

\section{(a) Motivation}

Conformal field theories (CFTs) are supposed to correspond to the non-trivial renormalization group (RG) fixed points of relativistic quantum field theories (QFTs). Such theories typically contain a number of scaling operators of dimension $\Delta<d$ (where $d$ is the space-time dimension), which, if added to the action, are relevant and drive the theory to what is, generically, a trivial fixed point. The points along this trajectory then correspond to a massive QFT. In general there is a multiplicity of such basins of attraction of the RG flows, but enumerating them and determining which combinations of relevant operators lead to which basins, and therefore to what kind of massive QFT, in general requires non-perturbative methods. This problem is equivalent to mapping out the phase diagram in the vicinity of the critical point corresponding to the CFT.

Another way of characterizing these massive theories is through the analysis of the possible boundary states of the CFT. Imagine the scenario in which the relevant operators are switched on in only a half-space, say $x_{0}<0$. This will then appear as some boundary condition on the CFT in $x_{0}>0$. However the boundary conditions themselves undergo RG lows, with fixed points corresponding to so-called conformal boundary conditions. Therefore on scales $\sim M^{-1}$, where $M$ is the mass scale of the perturbed theory, the correlations near the boundary should be those of a conformal boundary condition, deformed by irrelevant boundary operators.

Thus an important problem in all these cases is to determine to which conformal boundary condition a particular combination of bulk operators should correspond. In this talk I describe a simple prescription for doing this, based on a variational approach. A longer account appears in Ref. [5].

More specifically, we consider the action of deformed CFT with hamiltonian

$$
\hat{H}=\hat{H}_{C F T}+\sum_{j} \lambda_{j} \int \hat{\Phi}_{j}(x) d^{d-1} x,
$$

${ }^{4}$ This section was authored by John Cardy from the Department of Physics, University of California Berkeley, Berkeley, CA 94720, USA and All Souls College, University of Oxford, Oxford OX1 4AL, UK. The support for this section comes in part from the Simons Foundation and from the Perimeter Institute for Theoretical Physics. Research at the Perimeter Institute is supported by the Government of Canada through the Department of Innovation, Science and Economic Development and by the Province of Ontario through the Ministry of Research and Innovation. 
where the $\left\{\Phi_{j}\right\}$ are a set of relevant operators, and a variational state

$$
\left|\left\{\alpha_{a}\right\},\left\{\tau_{a}\right\}\right\rangle=\sum_{a} \alpha_{a} e^{-\tau_{a} \hat{H}_{C F T}}|a\rangle,
$$

where the sum over all possible conformal boundary states, and minimize

$$
\lim _{L \rightarrow \infty} \frac{1}{L^{D}} \frac{\left\langle\left\{\alpha_{a}\right\},\left\{\tau_{a}\right\}\left|\hat{H}_{C F T}+\sum_{j} \lambda_{j} \int \hat{\Phi}_{j}(x) d^{d-1} x\right|\left\{\alpha_{a}\right\},\left\{\tau_{a}\right\}\right\rangle}{\left\langle\left\{\alpha_{a}\right\},\left\{\tau_{a}\right\} \mid\left\{\alpha_{a}\right\},\left\{\tau_{a}\right\}\right\rangle},
$$

with respect to the $\left\{\alpha_{a}\right\}$ and $\left\{\tau_{a}\right\}$.

Eq. (2.1) simplifies because both terms are diagonal in the basis of physical boundary states, and they have a prescribed dependence on the $\left\{\tau_{a}\right\}$. Thus. for each $a$, we need to minimize an expression of the form

$$
E_{a}=\frac{D \sigma_{a}}{\left(2 \tau_{a}\right)^{D+1}}+\sum_{j} \lambda_{j} \frac{A_{a}^{j}}{\left(2 \tau_{a}\right)^{\Delta_{j}}},
$$

with respect to $\tau_{a}$, and then choose the $a$ which gives the absolute minimum. Here $\sigma_{a}$ is the universal coefficient of the Casimir energy per unit length with boundary conditions $a$, and $A_{a}^{j}$ the universal coefficient of the 1-point function $\left\langle a\left|\Phi_{j}\right| a\right\rangle$.

\section{(b) Results in two dimensions}

In this case, $\sigma_{a}=c / 12$, where $c$ is the central charge, and for the minimal models [6]

$$
A_{a}^{j}=\frac{S_{a}^{j}}{S_{a}^{0}}\left(\frac{S_{0}^{0}}{S_{0}^{j}}\right)^{1 / 2} \text {. }
$$

where $S_{a}^{j}$ are the known elements of the modular $S$-matrix of the CFT. This allows explicit calculations. There are several general features of the solutions.

(i) By its nature the method always gives the correct scaling of the energy with the coupling constants.

(ii) For a prescribed $a$, there is always some combination of couplings $\left\{\lambda_{j}\right\}$ for which this gives the minimum energy. Thus all boundary states correspond to an RG sink, and they all have a finite basin of attraction.

(iii) For any combination of couplings, there is always at least one $a$ for which a minimum occurs for finite $\tau_{a}$. This unfortunately rules out the possibility of the approximation correctly describing RG flows to another non-trivial CFT. Instead, in these cases, there are always at least two degenerate minima, indicating phase coexistence rather than a diverging correlation length. However the coexisting phases are physically sensible.

(iv) In known examples of integrable perturbations, where there is a higher degeneracy of RG sinks than expected on purely physical grounds, the approximation appears to reproduce this.

An alternative criterion, involving comparing overlaps between numerical approximations to the actual ground and different boundary states, has been suggested by Konechny [7]. From a numerical point of view our approach cannot be competitive with earlier methods such as the truncated conformal space approach [8,9], but it is much simpler and moreover gives new insight into the physical relationship between conformal boundary states and ground states of gapped theories.

\section{RGboundaries and interfaces in Ising field theory ${ }^{5}$}

This section is based on [10] where the details can be found. Here we only sketch the main idea as applied to the Ising field theory (IFT). The critical Ising model is a conformal field ${ }^{5}$ This section was authored by Anatoly Konechny from the Department of Mathematics at Heriot-Watt University, Edinburgh, UK and the Maxwell Institute for Mathematical Sciences, Edinburgh, UK. 
theory described in terms of free massless fermions $\psi, \bar{\psi}$. It has two relevant operators related to temperature and magnetic field. The IFT can be defined as a perturbed critical Ising model:

$$
S_{\mathrm{IFT}}=\frac{1}{2 \pi} \int(\psi \bar{\partial} \psi+\bar{\psi} \partial \bar{\psi}+i m \bar{\psi} \psi) d^{2} x+h \int \sigma d^{2} x .
$$

It is known that RG flows for all real values of the couplings $m$ and $h$ end up in a massive theory. In the far infrared only the vacuum state survives in the spectrum. If we, following the general idea of [11], perturb the critical theory only on a half space then in the far infrared we obtain a conformal boundary condition which we call an RG boundary. Moreover, if we put both (perturbed and unperturbed) theories on a cylinder than the asymptotic vacuum of the massive theory will be described as a conformal boundary state of the unperturbed theory. In the critical Ising model there are only three basic conformal boundary conditions that correspond to free of fixed boundary spins. Direct sums of these 3 basic conditions are also possible. The space of all RG flows corresponding to (3.1) then breaks up into a finite number of domains labeled by RG boundaries. We can think of these domains as infrared phases of the massive theories.

As generic flows correspond to non-integrable QFTs we use the numerical techniques of Truncated Conformal Space approach (TCSA) and Truncated Free Fermion Space Approach (TFFSA) of [12] to chart out the above domains. Calculating numerically the ratios of low conformal weight components of the vacuum vector we obtained a complete description of the assignment of RG boundaries to RG flows. The same answers were also independently obtained in [5] using an analytic approach based on a variational method.

We also explored RG flows with purely imaginary magnetic field. In this case there is a flow that ends up in a non-trivial infrared fixed point descried by Yang-Lee CFT. For this flow perturbing the critical Ising model on a half plane results in a conformal interface between the critical Ising and Yang-Lee CFTs. It is known due to [13] that there are only 12 such conformal interfaces that makes this flow particularly attractive to study. Combining numerical results with symmetry considerations and exactly solvable boundary flows we arrived to a conclusion that the flow in the space of interfaces is non-convergent in this case. Although the theory arrives to a fixed point the interface (that represents how the infrared states sit in the ultraviolet theory) perpetually oscillates never approaching any single conformal interface. This is possible due to the lack of unitarity.

\section{Double trace interfaces ${ }^{6}$}

\section{(a) Double trace perturbation}

For a scalar operator $\varphi$ on $d+1$ dimensional AdS space, with mass $-\frac{d^{2}}{4} \leq m \leq-\frac{d^{2}}{4}+1$, the expansion

$$
\phi(X) \sim \psi_{+}(x) \rho^{\Delta_{+}}+\psi_{-}(x) \rho^{\Delta_{-}}, \quad \rho \rightarrow 0
$$

close to the boundary $\rho=0$ of $A d S_{d+1}$ admits two different unitary boundary CFTs [14]: Either we take $\Delta_{+}=\frac{d}{2}+\nu$ with $\nu^{2}=\frac{d^{2}}{4}+m^{2}$ as the conformal dimension of the dual CFT scalar operator $\varphi=\varphi_{+}-$in which case $\psi_{+}(x)$ is proportional to the one-point function $\left\langle\varphi_{+}\right\rangle$in the presence of the source $\psi-$ or we pick $\Delta_{-}=\frac{d}{2}+\nu$ as the dimension. The two choices lead to different CFTs. $C F T_{-}$, corresponding to the choice $\Delta_{-}$, serves as the UV fixed point of an RG flow triggered by the relevant "double trace" operator $\varphi_{-}^{2}$, obtained (for large $N$ ) as the operator of lowest nontrivial dimension in the $\varphi_{-} \times \varphi_{-}$OPE. The RG flow takes the theory in the IR to $C F T_{+}$, the theory corresponding to the choice $\Delta_{+}$. 


\section{(b) Bulk Green's function and CFT correlators}

In order to study ${ }^{7}$ the RG interface [11] between $C F T_{-}$and $C F T_{+}$, we consider the Euclidean bulk, i.e. hyperbolic space $H_{d+1}$, in the Janus-type [16] coordinates

$$
d s_{H^{d+1}}^{2}=\frac{d z^{2}}{4 z^{2}(1-z)^{2}}+\frac{d s_{H^{d}}^{2}}{4 z(1-z)},
$$

where the radial coordinate $z$ runs from 0 to 1 . This choice of global coordinates foliates $H_{d+1}$ into slices of $H_{d}$, where the slice at any value of the coordinate $z$ meets the slice at $1-z$ at its conformal boundary. For the scalar bulk field, we impose $\Delta_{-}$boundary conditions at $z=0$, and $\Delta_{+}$boundary conditions at $z=1$. The interface is then located at the asymptotic intersection of the two $H^{d}$ patches covering the boundary of $H^{d+1}$.

The scalar Green's function in the bulk must then fall off as $G\left(z, x ; z^{\prime}, x^{\prime}\right) \sim z^{\Delta-}$ for $z \rightarrow 0$ (such that $x$ gives a point in $\mathcal{A}_{-}$), and as $G\left(z, x ; z^{\prime}, x^{\prime}\right) \sim(1-z)^{\Delta_{+}}$at $z \rightarrow 1$ (where $x$ is in $\mathcal{A}_{+}$). In the coordinates (4.2), the Green's function can be found from harmonic analysis. Starting from eigenfunctions $\psi_{s}$ of the Laplacian $-\nabla_{H^{d}}$ on the slices $H^{d}$, one may separate variables and write the bulk scalar field as a product $\phi(z, x)=\int d \mu(s) \psi_{s}(x) \Phi(s, z)$, where $d \mu(s)$ is an appropriate spectral measure. The space of solutions for the radial component $\Phi$ is two-dimensional, but in order to satisfy the boundary conditions ( $a$ at $z=0$ and $b$ at $z=1$, where $a, b= \pm$ ) we may use two different bases $\Phi_{L}^{a}$ and $\Phi_{R}^{b}$ with definite asymptotics

$$
\Phi_{L}^{a} \sim z^{\Delta_{a}} \quad(z \rightarrow 0), \quad \Phi_{R}^{b} \sim(1-z)^{\Delta_{b}}(z \rightarrow 1) .
$$

The solutions involve hypergeometric functions ${ }_{2} F_{1}$, and a change between the bases $\Phi_{L}^{a}$ and $\Phi_{R}^{b}$ can be obtained from Kummer's relations of ${ }_{2} F_{1}(z)$ to ${ }_{2} F_{1}(1-z)$. The bulk Green's function is then found from a suitable ansatz involving the solutions of the bulk scalar field equation, which manifestly satisfies the correct asymptotics. One finds that $G^{a b}\left(z, x ; z^{\prime}, x^{\prime}\right)$, with boundary condition $a$ for $z \rightarrow 0$ and $b$ for $z \rightarrow 1$, is of the form

$$
G^{a b}\left(z, x ; z^{\prime}, x^{\prime}\right)=\int_{0}^{\infty} d \sigma \mathcal{A}_{\sigma}^{a b} J_{\sigma}\left(x, x^{\prime}\right) \Phi_{L}^{a}\left(\sigma, \min \left(z, z^{\prime}\right)\right) \Phi_{R}^{b}\left(\sigma, \max \left(z, z^{\prime}\right)\right),
$$

where $\sigma$ is a continuous part of the label $s, \mathcal{A}_{\sigma}^{a b}$ is a constant involving Kummer's connection coefficients, $J_{\sigma}$ is a particular combination of products $\psi_{s}(x) \bar{\psi}_{s}\left(x^{\prime}\right)$ at fixed $\sigma$, and we use the fact that $\Phi_{L, R}$ actually only depend on $\sigma$. Specifying to the case $(a, b)=(-,+)$, the bulk-boundary propagator can be obtained as the limit

$$
K^{-+}\left(z, x ; x^{\prime}\right)=\frac{1}{d-2 \Delta_{+}} \lim _{z^{\prime} \rightarrow 1}\left(4 z^{\prime}\left(1-z^{\prime}\right)\right)^{-\Delta_{+} / 2} G^{-+}\left(z, x ; z^{\prime}, x^{\prime}\right),
$$

where $x^{\prime}$ is in $\mathcal{A}^{+}$. In the expression for the bulk-boundary propagator, the integration over $\sigma$ can be performed explicitly. Moving the bulk insertion at $(z, x)$ to the boundary, standard expansion methods then yield the scalar two-point correlation functions. In this way, we obtain the (flatspace) conformal correlation function

$$
\left\langle\varphi_{+}(x) \varphi_{+}\left(x^{\prime}\right)\right\rangle=\frac{1}{\left|x-x^{\prime}\right|^{2 \Delta_{+}}}\left(1+B \xi^{\Delta_{+}}{ }_{2} F_{1}\left(\begin{array}{c}
d / 2, \Delta_{+} \\
\Delta_{+}+1
\end{array} \mid-\xi\right)\right),
$$

where the interface is planar and situated at $y=0$ for $x=(\vec{x}, y), \xi=\left(x-x^{\prime}\right)^{2} /\left(4 y y^{\prime}\right)$ is the conformal cross ratio, and

$$
B=\frac{\Gamma(d / 2) \Gamma(\nu+1)}{\Gamma\left(\Delta_{+}+1\right)} \frac{\sin (\pi \nu)}{\pi} .
$$

We also obtain, in the same conformal frame as in (4.6),

$$
\left\langle\varphi_{-}(x) \varphi_{+}\left(x^{\prime}\right)\right\rangle=\sqrt{\frac{\sin (\pi \nu)}{\pi \nu}} \frac{\Gamma(d / 2)}{\sqrt{\Gamma\left(\Delta_{+}\right) \Gamma\left(\Delta_{-}\right)}} \frac{(-\xi)^{-\frac{d}{2}}}{\left(2 y^{\prime}\right)^{\Delta_{+}}(-2 y)^{\Delta_{-}}},
$$

${ }^{7}$ Details for the following computation can be found in [15] 
The $\varphi_{+} \times \varphi_{+}$OPE yields a conformal block decomposition into (quasi-)primary contributions ( [17], see also C. Kristjansen's contribution elsewhere in this review). The result (4.6) implies that the OPE contains primary operators of dimension $\Delta_{n}=2 \Delta_{+}+2 n$ for $n \in \mathbb{N}_{0}$, which we can identify as the double trace operators of $\varphi_{+}$descendents, with OPE coefficients

$$
C_{++}{ }^{n} B_{n}{ }^{0}=\frac{\sin (\pi \nu)}{\pi} \frac{\Gamma\left(\frac{d}{2}\right) \Gamma(\nu+n+1)}{n ! \Gamma\left(\Delta_{+}+n+1\right)} \frac{(\nu)_{n}\left(\Delta_{+}\right)_{n}}{\left(\Delta_{+}+\nu+n\right)_{n}} .
$$

In this equation, $C_{++}{ }^{n}$ is the OPE coefficient for the primary operator of dimension $\Delta_{n}$, and $B_{n}{ }^{0}$ is the coefficient of the interface identity operator when the insertion is close to the interface. From (4.8), we deduce that the bulk-to-defect OPE of $\phi_{+}$contains primary defect operators of dimension $\Delta_{\alpha}=\frac{d}{2}+\alpha, \alpha \in \mathbb{N}_{0}$, where the squares of the OPE coefficients are

$$
\left(B_{+}{ }^{\alpha}\right)^{2}=\frac{\sin (\pi \nu)}{\pi \nu} \frac{\alpha !}{(2 \alpha) !} \frac{\Gamma\left(\frac{d}{2}\right)}{\Gamma\left(\Delta_{+}\right)} \frac{\Gamma(\nu+1+\alpha)}{(1-\nu)_{\alpha}} .
$$

These operators form the single-trace part of the full interface spectrum at large $N$, which can be anticipated by methods explained in [18].

\section{(c) Interface free energy}

The contribution of the interface to the free energy of the system can be calculated from considering the product theory $C F T_{+} \otimes C F T_{-}$and inserting a product of the interface in such a way that the two factors are linked. Comparing to the product CFT without any interface we have

$$
2 F_{\text {interface }}=F_{+-}+F_{-+}-F_{++}-F_{--}=\frac{1}{2} \log \left(\frac{\operatorname{det} \mathcal{D}^{+-} \operatorname{det} \mathcal{D}^{-+}}{\operatorname{det} \mathcal{D}^{++} \operatorname{det} \mathcal{D}^{--}}\right),
$$

which is a UV-finite expression. Regularising the IR divergence [19] leads to

$$
\frac{d}{d \nu} 2 F_{\text {interface }}\left(=\frac{d}{d \nu} \log g^{2}\right)=-\nu \frac{\cos \pi \nu}{\cos \frac{\pi d}{2}} \frac{\Gamma\left(\Delta_{+}\right) \Gamma\left(\Delta_{-}\right)}{\Gamma(1+d)} .
$$

Since the sphere in two dimensions is conformally equivalent to the cylinder, this yields the $g$ factor of the interface for $d=2$.

\section{(d) Conformal field theory tests}

The reason for the simplicity of the interface and the corresponding RG flow is the large $N$ limit (see also the contributions by A. Bissi and C. Kristjansen in this review). In the gravitational bulk, the limit in particular suppresses quantum gravity fluctuations. On the CFT side, large $N$ theories are "generalised free", which means in particular that correlation functions are obtained from Wick contractions, and also that the diverging parts of the OPEs of the scalar and the double trace operators are of the particular form

$$
\varphi \times \varphi \sim 1+C \varphi^{2}, \quad \varphi^{2} \times \varphi^{2} \sim 1+2 C \varphi^{2}
$$

for some constant $C$. From this simple behaviour, the constants in (4.6) and (4.8) can be verified perturbatively, since the renormalised coupling constant of the double trace deformation is of order $\nu$ for small $\nu$. This observation also entails that the expansion in $\nu$ of our bulk results organises the loop expansion of conformal perturbation theory, at least in an appropriate scheme.

In the special case of a two-dimensional boundary $d=2$, the higher spin/CFT correspondence [20] provides a testing ground for our results. In particular, the UV-IR operator overlaps can be obtained exactly on the CFT side, using the interface construction of [21]. The coefficients of (4.6) and (4.8) are precisely matched. 


\section{(e) Outlook}

The tools of harmonic analysis used to obtain the bulk results can be replaced by those suitable for mixed boundary value problems [22] in more complicated situations. In particular, our aim for the near future is to investigate the fusion process of the presented double trace interfaces by these methods.

\section{Boundary conformal field theory and topological phases of matter $^{8}$}

\section{(a) Introduction}

Boundary conformal field theory (BCFT) has played a pivotal role in modern theoretical physics, with its application ranging from problems in condensed matter physics such as the Kondo (impurity) problem, to high-energy physics, such as D-branes [23]. In this article, we give an overview of the use of BCFT (and defect CFT) in the context of topological phases of condensed matter.

Topological phases are fully gapped states of matter, which are (topologically) distinct from trivial states that can be written as product states. Specifically, we can distinguish the following two kinds of topological phases. Topologically-ordered (TO) phases are phases which support non-trivial anyonic excitations, and are characterized, for example, by non-trivial topological ground state degeneracy on spatial manifolds with non-trivial topology. Symmetry-protected topological (SPT) phases are phases which cannot be adiabatically deformed to a trivial product state in the presence of certain symmetry, although they do not support anyonic excitations, and are trivial once we do not take the symmetry into account. In the long wavelength limit, topological phases (of both kinds) are expected to be described by topological quantum field theories

\section{(b) Boundary CFT and topological phases}

It is noted that $\mathrm{BCFT}$ in $(1+1) \mathrm{d}\left(\mathrm{BCFT}_{2}\right)$ can be used to discuss properties of topological phases of matter both in $(1+1) \mathrm{d}$ and $(2+1) \mathrm{d}$. More specifically, the following connections between $\mathrm{BCFT}_{2}$ and topological phases in $(1+1)$ and $(2+1)$ dimensions have been discussed in the literature:

- $\mathrm{SPT}_{2} / \mathrm{BCFT}_{2}[24,25]$

(topological invariants and entanglement spectrum)

- $\mathrm{SPT}_{3} / \mathrm{BCFT}_{2}[26]$

(diagnosing anomalies of boundaries of bulk topological phases)

- $\mathrm{TO}_{3} / \mathrm{BCFT}_{2}$ [27-30]

(computation of various entanglement measures such as entanglement entropy, mutual information, negativity, etc.)

CFTs describe gapless states such as quantum critical points - why CFTs have something to do with topological phases, which are fully gapped? The basic reason is that CFTs can be in proximity to gapped phases (i) in the phase diagram or (ii) in space.

In Case (i), BCFT and SPT phases in the same spacetime dimensions are related, and this is the case for the $\mathrm{SPT}_{2} / \mathrm{BCFT}_{2}$ connection, More specifically, the connection can be made by considering a renomalization group (RG) domain wall [11] between an SPT phase and a CFT, which is adjacent to the SPT phase in the phase diagram. From the point of view of the CFT, the SPT phase serves as a conformal invariant boundary, and hence the RG domain wall is described

${ }^{8}$ This section was authored by Shinsei Ryu from the James Franck Institute and Kadanoff Center for Theoretical Physics at the University of Chicago, Chicago, Illinois 60637, USA . This work was supported in part by the National Science Foundation grant DMR-1455296. 
by a BCFT. It is then possible, from the properties of the corresponding boundary state, ${ }^{9}$ to extract the group cohomology class - This is consistent with the known classification of $(1+1) \mathrm{d}$ SPT phases protected by unitary on-site symmetry $G$, which is given by $H^{2}(G, U(1))$ [31]. ${ }^{10}$ It was also noted that if one considers the entanglement spectrum of an SPT phase, which is in the vicinity of a critical point, the RG domain wall naturally appears.

The connection of type (ii) connecting topological phases and CFT in one-lower dimensions is commonly called the bulk-boundary correspondence, and applies to $\mathrm{SPT}_{3} / \mathrm{BCFT}_{2}$ and $\mathrm{TO}_{3} / \mathrm{BCFT}_{2}$ connections. As known in the context of the quantum Hall effect, topologically non-trivial bulk phases are necessarily accompanied by gapless edge states, which suffer from a quantum anomaly of certain kind (gauge and/or modular anomalies). For a bulk SPT phase, the corresponding edge theory has a modular anomaly if the symmetry of the SPT phase is gauged or orbifolded [32]. The modular anomaly can be diagnosed, alternatively, by considering possible boundaries (boundary states) in the edge theory. If the bulk is a non-trivial SPT phase, it should not be possible to make a boundary to the edge theory, since it lives already on a boundary of a bulk system in one higher dimensions, as far as the symmetry of the SPT is strictly enforced. This is the $\mathrm{SPT}_{3} / \mathrm{BCFT}_{2}$ connection.

Finally, in the $\mathrm{TO}_{3} / \mathrm{BCFT}_{2}$ connection, boundary states (Ishibashi boundary states) appear when we partition a (2+1)-dimensional topological liquid into two or more parts, and consider the reduced density matrix for a subregion of the total system by tracing out its complement. The explicit expression of the density matrix can be used to compute the various entanglement measures, such as the von Neumann and Renyi entanglement entropy, the mutual information, and the entanglement negativity. There are contributions to these quantities, which can be used to indentiy topological order, e.g., the topological entanglement entropy.

\section{(c) Boundary states as gapped ground states}

All these applications of BCFTs to topological phases share one common idea: boundary states as gapped $(1+1)$ states $[5,33,34]$.

To be more concrete, let us start from a CFT described by the (Euclidean) action $S_{*}$. One can then perturb $S_{*}$ by adding a perturbation: $S=S_{*}-\lambda \int d^{2} x \mathcal{O}(x)$. In the context of the SPT/BCFT connections, we assume the perturbation respects the symmetry of the SPT phase in question. The perturbation, when releyant, may drive the system into a gapped phase without breaking the symmetry. Alternatively, the theory may flow to a different critical point, or may break the symmetry. In the current context, we disregard these possibilities; In the entanglement spectrum setup considered in [24,25], these scenarios are known not to occur.

One may then ask what the ground state of the gapped theory is; how does the ground state look like within the Hilbert space of the CFT? It is postulated that it is given by a boundary state of the CFT.

We should note that, in general, it may not be easy to determine which relevant perturbation leads to which boundary state, or which boundary state is obtained by which relevant perturbation. In the context of $(1+1) \mathrm{d}$ SPT phases, however, one can identify boundary states by assigning/computing an SPT topological invariant. More specifically, let us consider a (1+1)d SPT phase protected by unitary on-site symmetry $G$. Following the above discussion, we expect that ground states of $(1+1)$ d SPT phases may be represented by boundary states in a CFT. This CFT is not unique, but should be proximate to the SPT phase; The CFT is either a critical point separating the SPT phase from other gapped phases or it represents a critical intermediate phase proximate to the SPT phase. Then, in [25], it is claimed that $g|B\rangle_{h}=\varepsilon_{B}(g \mid h)|B\rangle_{h}$. Here, $g, h \in G, g h=h g$, and $|B\rangle_{h}$ is the boundary state in the $h$-twisted sector. The set of phases $\varepsilon_{B}(g \mid h)$ is the topological invariant of the SPT phase, and identical to the group cohomology

${ }^{9}$ By construction, this boundary state preserves the symmetry of the SPT phase.

${ }^{10}$ By on-site, we mean group elements $g$ in $G$ are all non-spatial symmetry operation. Furthermore, if the total Hilbert space $\mathcal{H}$ is decomposed into the local Hilbert space $\mathcal{H}_{i}$ defined for each lattice site as $\mathcal{H}=\mathcal{H}_{1} \otimes \cdots \otimes \mathcal{H}_{N}$, then the on-site symmetry operation $g$ is also factorized as $g=g_{1} \otimes g_{N}$ where $g_{i}$ acts exclusively on $\mathcal{H}_{i}$. 
phase. Furthermore, in [25], the topological invariant $\varepsilon_{B}(g \mid h)$ is related to the symmetry-protected degeneracy of the entanglement spectrum of SPT phases.

\section{(d) Conclusion}

(1+1)-dimensional boundary/defect CFT can provide a convenient tool to diagnose and characterize topological phases of matter both in $(1+1)$ and $(2+1)$-dimensions. It would be interesting to explore if there is a higher dimensional analogue of the approaches discussed in this article. In this regard, it should be noted that the surfaces theories of $(3+1)$-dimensional SPT phases can be, when they respect the same symmetry as the bulk, either gapless or gapped with topological order. It is also worth mentioning that, in addition to boundary states, cross cap states are also useful to study topological phases protected by and enriched with an orientation reversing symmetry [35].

\section{Quantum Dot in Interacting Environments ${ }^{11}$}

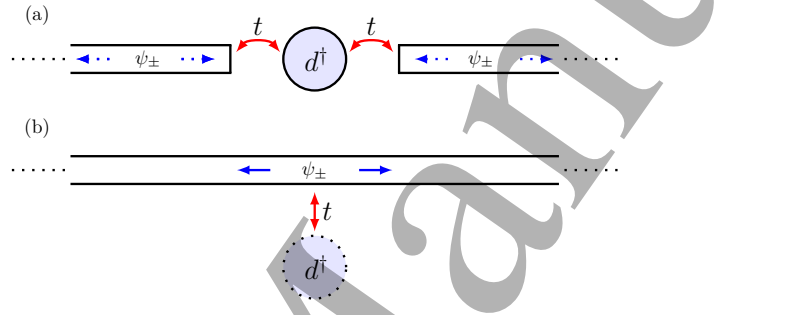

Figure 1. We consider two geometries of Luttinger dot system; (a) embedded and (b) side-coupled. The embedded geometry also includes a Coulomb interaction between the dot and leads. Once unfolded the side-coupled and embedded geometries are the same but with the latter containing non local interactions.

Coupling a quantum impurity to an interacting one dimensional lead produces some of the most striking phenomena of low dimensional physics. A simple backscattering impurity is known to cause the wire to be split if the interactions are repulsive while a junction between two leads can lead to perfect conductance in the presence of attractive interactions [36]. More interesting still are scenarios in which the impurity has internal degrees of freedom. These allow for richer and more exotic phases to appear [37]. Among these, systems of quantum dots coupled to interacting leads have attracted much attention [36,38-47]. The low energy description of the leads is typically given by Luttinger liquid theory which is the effective low energy description of a large number of interacting systems [37,48]. Here the individual electrons are dissolved and the excitations are bosonic density modes. In contrast, the relevant degrees of freedom on the dot are electronic. A competition ensues between the tunneling from lead to dot which is carried out by electrons and the energy cost of reconstituting an electron from the bosons in the lead.

Such systems are readily achievable in many experimental settings allowing for confrontation of theory with experiment. Luttinger liquids provide the effective description of carbon nano tubes [49,50], fractional quantum Hall edges [51-53], cold atomic gases [54-57] or ${ }^{4} \mathrm{He}$ flowing through nano pores $[58,59]$ to name but a few. Additionally they are known to describe tunneling processes in higher dimensional resistive leads $[60,61]$ and more generally are the archetype of a non-Fermi liquid. Luttinger liquid-quantum dot systems have successfully been realized in a number of experiments $[62,63]$. These realize the embedded geometry, see Fig. 1(a) of a dot placed between two otherwise disconnected leads. Measurement of the conductance has revealed interesting non-Fermi liquid scaling as well as Majorana physics.

${ }^{11}$ This section was authored by Natan Andrei from the Department of Physics at Rutgers University, Piscataway, New Jersey, USA 08854. This work was funded by NSF Grant DMR 1410583. 

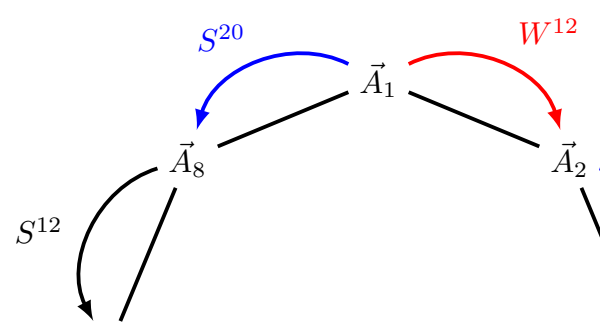

Figure 2. (Color Online) The amplitudes are related by a consistent set of $S$-matrices.

We study the theory of these systems which consist of a quantum dot attached to an interacting lead, a Luttinger liquid, the attachment being either in the embedded or the side-coupled geometry. The Hamiltonian of a Luttinger liquid is given by,

$$
H_{\mathrm{LL}}=-i \int d x\left(\psi_{+}^{\dagger} \partial_{x} \psi_{+}-\psi_{-}^{\dagger} \partial_{x} \psi_{-}\right)+4 g \int d x \psi_{+}^{\dagger}(x) \psi_{-}^{\dagger}(x) \psi_{-}(x) \psi_{+}(x)
$$

where $\psi_{ \pm}^{\dagger}$ are right and left moving fermions which interact with a point like interaction of strength $g$ [37]. For the side-coupled geometry we have $x \in[-L / 2, L / 2]$ while for the embedded geometry we take two Luttinger liquids restricted to $x \in[-L / 2,0]$ and $x \in[0, L / 2]$. The Luttinger model is typically presented in a bosonized form,

$$
H_{L L}=\frac{1}{4 \pi} \int\left(K(\nabla \varphi)^{2}+\frac{1}{K} \Pi^{2}\right) d x
$$

where $\varphi(x)$ and $\Pi(x)$ are canonically conjugate bosonic fields and $K$ is related to the coupling $g$,

$$
K= \begin{cases}1+\frac{\phi}{\pi} & \text { side-coupled } \\ \frac{1}{1-\frac{\phi}{\pi}} & \text { embedded }\end{cases}
$$

where $\phi=-2 \arctan (g)$. Thus repulsive interaction in the wire, $g>0$, corresponds to $K<1$, attractive interaction to $K>1$, and no interaction to $K=1$.

The quantum dot is modelled by a resonant level with energy $\epsilon_{0}$ described by,

$$
H_{\mathrm{dot}}=\epsilon_{0} d^{\dagger} d,
$$

coupled to Luttinger liquid via a tunnelling term,

$$
H_{t}=\frac{t}{2}\left(\psi_{+}^{\dagger}(0)+\psi_{-}^{\dagger}(0)\right) d+\text { h.c }
$$

The fully interacting Hamiltonian $H=H_{L L}+H_{t}+H_{\text {dot }}$ can be studied exactly by means of the Bethe Ansatz approach which allows the construction of the full set of exact eigenstates , and spectrum, and from it the determination of the ground state $(T=0)$ and thermodynamic $(T>0)$ properties of the system. It is important to note here that a novel type of Ansatz was necessary, 
distinct from that which has been typically used for quantum impurity models $[64,65]$. As the problem contains both forward and back scattering we must formulate it in an in-out basis with the configuration space being partitioned in regions labelled by both the order of the particles and by their closeness to the origin. One thus partitions the configuration space not to $N$ ! regions (orderings of particles) as is conventionally done but to $2^{N} N$ ! regions. The large degeneracy present in the bulk system due to the linear derivative is then used to find a consistent set of wave functions [66], the consistency assured by a generalised Yang-Baxter relation. The $2^{2} 2 !=8$ amplitudes, for example, describing 2-particle configuration space are related by a consistent set $S$-matrices deduced from the Hamiltonian and requirement of uniqueness, see Fig. 2.

Having obtained a consistent and complete set of eigenfunctions one proceeds to impose periodic boundary conditions which lead in turn to a set of equations that determine the momenta $k_{j}$ and the energies, $E=\sum_{j} k_{j}$. For details see [69]. Here we present some results for the dot occupation $n_{d}=\left\langle d^{\dagger} d\right\rangle$ as a function of the level energy $\epsilon_{0}$ and the temperature $T$, both expressed with respect to the width $\Gamma$ which serves as the strong coupling scale of the model: by,

The ground state dot occupation $n_{d}$ for attractive interaction is given for for $\epsilon_{0}<\Gamma$ or $\epsilon_{0}>\Gamma$

$$
n_{d}= \begin{cases}\frac{1}{2}-\left[\sum_{n=0}^{\infty} a_{n}\left(\frac{\epsilon_{0}}{\Gamma}\right)^{2 n+1}+b_{n}\left(\frac{\epsilon_{0}}{\Gamma}\right)^{(2 n+1) /(K-1)}\right] \\ \sum_{n=0}^{\infty} c_{n}\left(\frac{\Gamma}{\epsilon_{0}}\right)^{n+1} & \text { for } \Gamma<\epsilon_{0}\end{cases}
$$

while for repulsive interaction we have,

$$
n_{d}=\left\{\begin{array}{lr}
\frac{1}{2}-\sum_{n=0}^{\infty} a_{n}\left(\frac{\epsilon_{0}}{\Gamma}\right)^{2 n+1} & \text { for } \Gamma>\epsilon_{0} \\
\sum_{n=0}^{\infty} c_{n}\left(\frac{\Gamma}{\epsilon_{0}}\right)^{n+1}+b_{n}\left(\frac{\Gamma}{\epsilon_{0}}\right)^{(2 n+1) /(1-K)}
\end{array}\right.
$$

where $a_{n}, b_{n}$ and $c_{n}$ are constants. See FIG 3 and 4 .

Beginning with the attractive case, $K>1$ we see that at low energy, $\epsilon_{0}<\Gamma$ the system is strongly coupled with the dot becoming hybridized with the bulk. At the low energy fixed point $\left(\epsilon_{0}=0\right)$ the dot is fully hybridized and has $n_{d}=1 / 2$. The leading term in the expansion about this is $\epsilon_{0} / \Gamma$ which indicates that the leading irrelevant operator has dimension 2 . We identify it as the stress energy tensor [67]. The next order term $\left(\epsilon_{0} / \Gamma\right)^{1 /(K-1)}$ is due to the backscattering which is generated at low energies but is irrelevant for $K>1$. At high energies, $\epsilon_{0}>\Gamma$, the system becomes weakly coupled with the fixed point $\left(\epsilon_{0} \rightarrow \infty\right)$ describing a decoupled empty dot, $n_{d}=0$. The expansion about this fixed point is in terms of integer powers indicating that the tunnelling operator $d^{\dagger} \psi(0)$ has dimension $1 / 2$. The first few terms of the expansion are plotted in Fig. 3 from which we see that the dot occupation is suppressed as a function of $\epsilon_{0}$ for $K>1$ as compared to the non interacting case due to the backscattering. For the repulsive case, $K<1$, we see again as for $K>1$, the dot is strongly coupled at low energy and weakly coupled at high energy with the same leading terms in the expansion about these points, however the term generated by the backscattering now appears in the expansion about the high energy fixed point. This stems from the fact that backscattering is relevant for $K<1$ and leads to an enhancement of the dot occupation as compared to the $K=1$ case, Fig. 4.

The following RG picture emerges for the side coupled dot: For all $K \in[0,2]$ the system flows from weak coupling at high energy to strong coupling at low energy. The low energy fixed point describes a dot which is fully hybridized with the bulk and has the fixed point occupation $n_{d}=1 / 2$. The hybridized dot then acts as a backscattering potential via co-tunnelling. The leading irrelevant operator which perturbs away from the fixed point is the stress energy tensor and results in odd integer powers of $\epsilon_{0} / \Gamma$ in the dot occupation. For $K>1$ the backscattering is irrelevant which gives rise to odd powers of $\left(\epsilon_{0} / \Gamma\right)^{1 /(K-1)}$ resulting in a suppression of the dot occupation at $\epsilon_{0}>0$. For $K<1$ on the other hand it is relevant and generates no other terms in the expansion. The high energy fixed point describes a decoupled dot which has $n_{d}=0$ for $\epsilon_{0} \rightarrow \infty$ or $n_{d}=1$ for $\epsilon_{0} \rightarrow-\infty$. By reducing the energy scale we flow away from the fixed point with the tunnelling operator $d^{\dagger} \psi_{ \pm}(0)$ which is the leading relevant operator and has dimension 

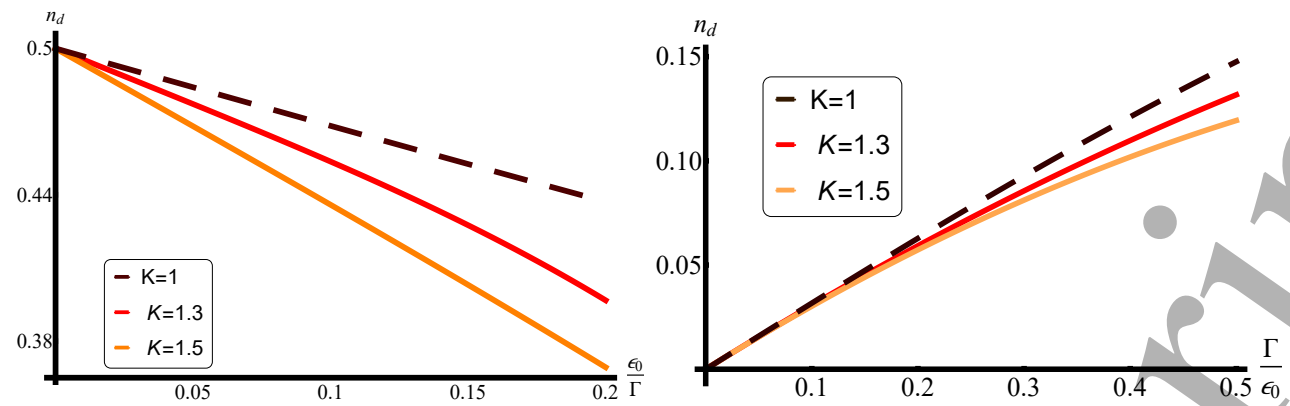

Figure 3. (Color Online). The dot occupation at small (left) and large (right) dot energy, $\epsilon_{0} / \Gamma$, for different values of $K>1$. The effect of attractive interactions is to suppress the dot occupation as compared to the non interacting case (dashed line). This effect becomes stronger for increasing $K$.

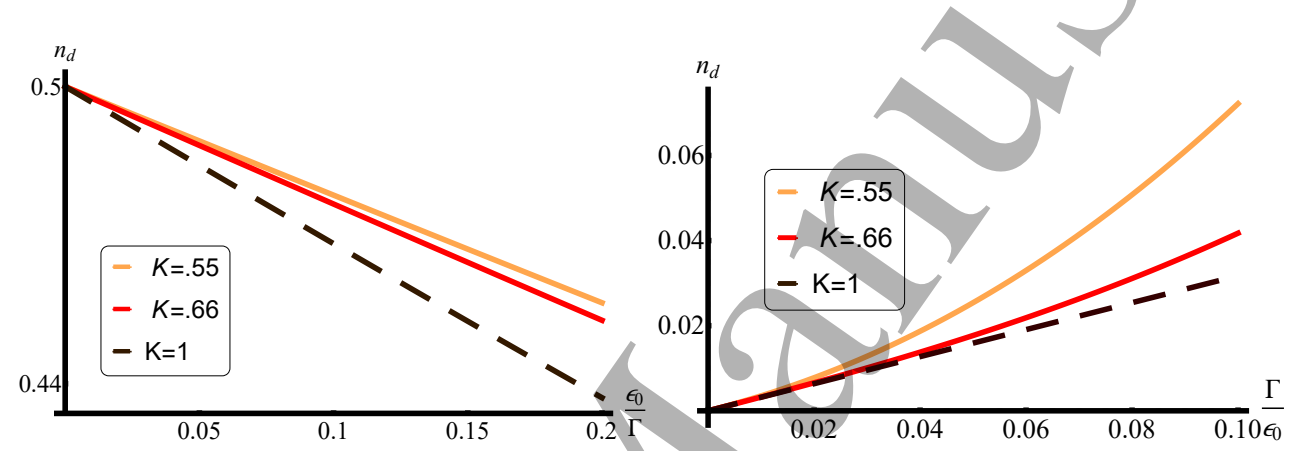

Figure 4. (Color Online). The dot occupation at small (left) and large (right) dot energy for different values of $K$. The effect of repulsive interactions $K<1$ is to enhance the dot occupation as compared to the non interacting case (dashed line) with the effect increasing as $K$ decreases.

$1 / 2$ as in the free model. This give rise to integer powers of $\Gamma / \epsilon_{0}$ in $n_{d}$. Additionally when $K<1$ backscattering is relevant and causes odd powers of $\left(\Gamma / \epsilon_{0}\right)^{1 /(1-K)}$ to appear resulting in an enhancement of the dot occupation .

Now turning to the study of the system at finite temperature we obtained the thermodynamic Bethe Ansatz equations following the approach of Yang-Yang and Takahashi. Using these we can check the RG picture we arrived at earlier using the ground state dot occupation still holds true at finite temperature. We find that the temperature again is measured with respect to the level width for the model $\Gamma$ which serves as the strong coupling scale. Thus the system is strongly coupled at low temperature $T \ll \Gamma$ and weakly coupled at high temperature $T \gg \Gamma$. We also obtained $g$-function of the model, defined to be the difference in the UV and IR entropy of the impurity

$$
g=S_{\mathrm{UV}}-S_{\mathrm{IR}}=\log 2+\frac{1}{2} \log \left(\frac{1}{K}\right) .
$$

This is always positive for the range of values considered in agreement with the requirement that as we move along the RG flow by lowering the temperature, massless degrees of freedom are integrated out. The first term comes from the charge degrees of freedom and corresponds to the entropy of a decoupled dot at high temperature which is fully hybridised at low temperature. The second term comes from the chiral degrees of freedom and is the same as for the Kane-Fisher model of a back scattering impurity [66,68]. Note however that although $g>0$, the second term which is due to the backscattering, is negative for $K>1$. This relative sign between the charge and chiral terms is related to the competition between the tunnelling and the backscattering. We see 


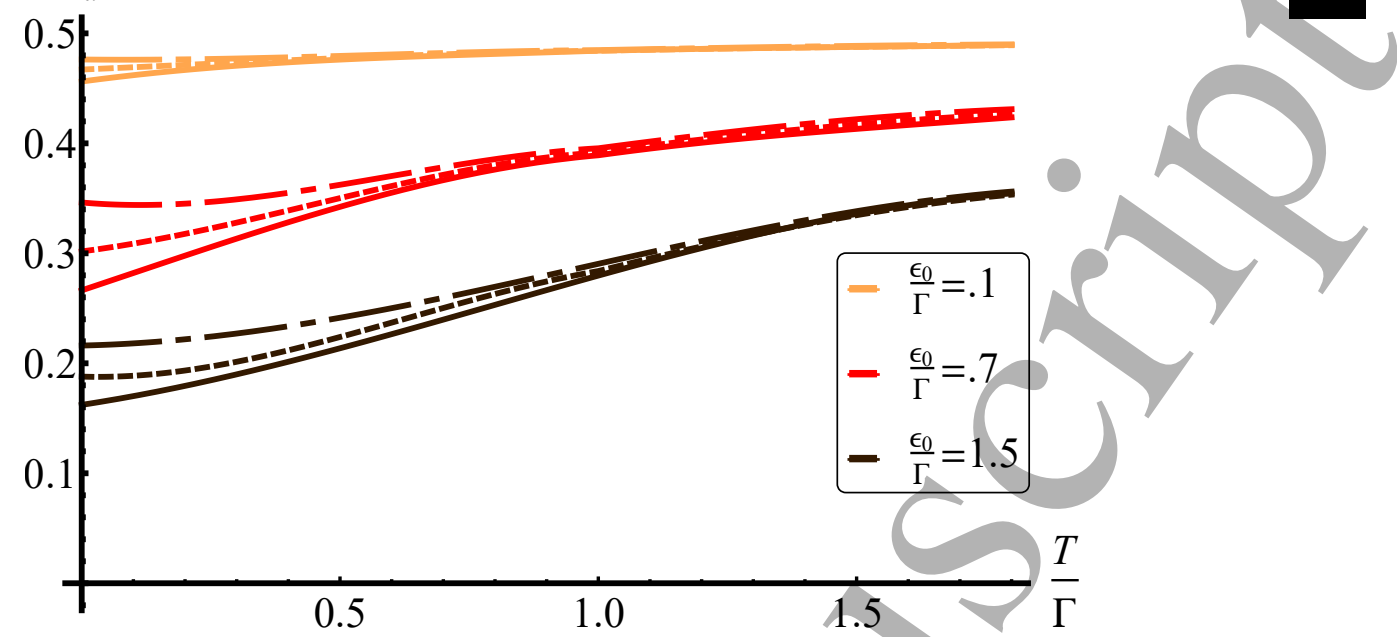

Figure 5. (Color Online): The dot occupation for fixed $\epsilon_{o} / \Gamma$ as a function of temperature. The interaction is taken to be $K=\frac{4}{3}$ (dot-dashed lines), $K=1$ (dashed lines) and $K=\frac{2}{3}$ (solid lines). We see the enhancement and suppression of the dot occupation for repulsive and attractive interaction with the effect most pronounced as the temperature is lowered.

from this that at high temperature the dot is decoupled and as $T$ is lowered it becomes hybridised with the dot whereupon it acts as a back scattering impurity. In the non interacting limit the $K \rightarrow 1$ this last term disappears and we recover the expected result.

We may go beyond the fixed point behaviour to get the leading order corrections and determine the specific heat. The low temperature specific heat is found to be

$$
C_{v} \sim \frac{T}{\Gamma}+\text { corrections }
$$

which agrees with the expectation that the irrelevant operator is the stress energy tensor. The corrections are still to be calculated but the exponents are expected to agree with those found earlier.

The lack of fine tuned parameters in the side-coupled model make it a good candidate for experimental realizations. Such a system may be created placing a quantum dot near a carbon nanotube, the edge of a quantum Hall sample or a topological insulator. The dot occupation can then be measured by means of a quantum point contact and compared to our results.

\section{A Holographic Kondo model ${ }^{12}$}

\section{(a) The Kondo model and its gravity dual}

The original model of Kondo [106] describes a SU(2) spin impurity interacting with a free electron gas. It is given by the Hamiltonian

$$
H=\frac{v_{F}}{2 \pi} i \psi^{\dagger} \partial_{x} \psi+\frac{v_{F}}{2} \lambda_{K} \delta(x) J \cdot S,
$$

with $v_{F}$ the Fermi velocity, $\psi$ the electron field, $J$ the electron current, $S$ the impurity spin and $\lambda_{K}$ the Kondo coupling. The Kondo Hamiltonian explains the logarithmic rise of the resistivity with decreasing temperature observed in metals with magnetic impurities: at low energies, the

${ }^{12}$ This section was authored by Johana Erdmenger from the Institute for Theoretical Physics and Astrophysics at JuliusMaximilians-Universität Würzburg, Am Hubland 97074 Würzburg, Germany. This section is based on joint work with Andy O'Bannon, Carlos Hoyos, Mario Flory, Max Newrzella, Jackson Wu and Ioannis Papadimitriou. 
impurity is screened by the electrons. From a theoretical perspective, this behaviour is due to the fact that the beta function of $\lambda_{K}$ is negative.

The holographic Kondo model of [107] differs from the original condensed matter model in that the ambient electrons are strongly coupled among themselves even before the interaction with the magnetic impurity is turned on. Moreover, the impurity is an $S U(N)$ spin with $N \rightarrow \infty$. The ambient degrees of freedom are dual to a gravity theory in an $\mathrm{AdS}_{3}$ geometry at finite temperature. The impurity degrees of freedom are dual to an $\mathrm{AdS}_{2}$ subspace. The dual gravity model corresponds to a holographic RG flow dual to a UV fixed point perturbed by a marginally relevant double-trace operator, which flows to an IR fixed point. In addition, in the IR a condensate forms, such that the model has some similarity to a holographic superconductor [108].

As found in $[109,110]$, the Kondo model simplifies considerably when the rank $N$ of the spin group is taken to infinity. In this limit the interaction term $J \cdot S$, which involves two vectors in spin space, reduces to a product $\mathcal{O O}^{\dagger}$ of a scalar operator $\mathcal{O}$ and its conjugate. $\mathcal{O}$ involves an electron $\psi$ and an auxiliary 0+1-dimensional fermion field $\chi$. The latter is introduced by writing the spin $S$ as a fermion bilinear. It is found that the operator $\mathcal{O}$ condenses below a critical temperature.

\section{(b) Gravity action: properties and applications}

The motivation for establishing a gravity dual of the Kondo model is twofold. First, it will provide a new application of gauge/gravity duality relevant to condensed matter physics. Second, it will provide a gravity dual of a well-understood condensed matter model with an RG flow, which may provide new insights into the duality's working mechanisms. Crucially, our holographic Kondo model will have some features that are distinctly different from the well-known field theory Kondo model described above. Most importantly, the 1+1-dimensional electron gas will be strongly coupled even before considering the interaction with the impurity. The model thus has some resemblance to a Luttinger liquid coupled to an impurity spin. Furthermore, in our model the $S U(N)$ spin symmetry will be gauged.

The holographic Kondo model of [107] is motivated by a D-brane construction in string theory involving D3-, D7- and D5-branes. However, a simpler, phenomenological gauge/gravity model captures the essential physics, namely the model with gravity action

$$
\begin{aligned}
S= & \frac{1}{8 \pi G_{N}} \int d z d x d t \sqrt{-g}(R-2 \Lambda)-\frac{N}{4 \pi} \int_{\mathrm{AdS}_{3}} A \wedge d A \\
& -N \int d x d t \sqrt{-g}\left(\frac{1}{4} f^{m n} f_{m n}+\left(D^{m} \Phi\right)^{\dagger}\left(D_{m} \Phi\right)-V(\Phi)\right) .
\end{aligned}
$$

Here, $z$ is the AdS holographic coordinate, $x$ is the spatial coordinate along the boundary and $t$ is time. The bulk defect dual to the impurity is at $x=0$. The first term is the Einstein-Hilbert action with negative cosmological constant $\Lambda$. The second term is a Chern-Simons term for the gauge field $A_{\mu}$ dual to the electron current $J^{\mu}$. We take $A_{\mu}$ to be Abelian, which implies that we have only one flavour (or channel) of electrons. $f_{m n}$ is the field strength of the defect Abelian gauge field $a_{m}$ with $m \in\{t, z\}$. Its time component $a_{t}$ is dual to the charge $\chi^{\star} \chi$, which at the boundary takes the value $Q=q / N$ with $q$ the dimension of the antisymmetric representation of the impurity spin. $D_{m}$ is a covariant derivative, $D_{m}=\partial_{m}+i A_{m} \Phi-i a_{m} \Phi$. For the complex scalar, we assume its potential takes the simple form $V\left(\Phi^{\dagger} \Phi\right)=M^{2} \Phi^{\dagger} \Phi$. We choose $M^{2}$ such that $\Phi^{\dagger} \Phi$ is a relevant operator in the UV. It becomes marginally relevant when perturbing about the UV fixed point. For the background geometry we take the solution to the gravity equations of motion the AdS BTZ black hole, i.e.

$$
d s_{\mathrm{BTZ}}^{2}=\frac{1}{z}\left(\frac{1}{h(z)} d z^{2}-h(z) d t^{2}\right), \quad h(z)=1-\frac{z^{2}}{z_{h}^{2}},
$$

where we choose units with AdS radius equal to one, $L=1$, and the horizon location $z_{h}$ is related to the temperature $T$ by $T=1 /\left(2 \pi z_{h}\right)$. The holographic dictionary for this model appears in table 


\begin{tabular}{|l|c|l|}
\hline Operator & & Gravity field \\
\hline Electron current $J$ & $\Leftrightarrow$ & Chern-Simons gauge field $A$ in $A d S_{3}$ \\
\hline Charge density $q=\chi^{\dagger} \chi$ & $\Leftrightarrow$ & 2d gauge field $a$ in $A d S_{2}$ \\
\hline Operator $\mathcal{O}=\psi^{\dagger} \chi$ & $\Leftrightarrow$ & 2d complex scalar $\Phi$ in $\mathrm{AdS}_{2}$ \\
\hline
\end{tabular}

Table 1. Field-operator map for the holographic Kondo model.

1. The charge $q$ measures the dimension of the totally antisymmetric spin representation and thus the number of impurity degrees of freedom. The equations of motion derived from the action (7.2) can be solved numerically. The results reveal that the operator $\mathcal{O}$ dual to the field $\Phi$ indeed condenses below a critical temperature. This leads to the expected screening of impurity degrees of freedom: the impurity charge indeed decreases as $T$ decreases below the critical temperature.

\section{(c) Impurity Entropy}

The concept of holographic entanglement entropy has proved to be an important ingredient to the holographic dictionary [111]. In general, the entanglement entropy is defined for two Hilbert spaces $\mathcal{H}_{A}$ and $\mathcal{H}_{B}$. In the AdS/CFT correspondence, it is useful to consider $A$ and $B$ to be two disjunct space regions in the CFT. Defining the reduced density matrix to be

$$
\rho_{A}=\operatorname{tr}_{B} \rho
$$

where $\rho$ is the density matrix of the entire space, the entanglement entropy is given by its von Neumann entropy

$$
S=-\operatorname{tr}_{A} \rho_{A} \ln \rho_{A}
$$

Ryu and Takayanagi [111] proposed the holographic dual of the entanglement entropy to be

$$
S=\frac{\operatorname{Area} \gamma_{A}}{4 G_{d+1}}
$$

where $G_{d+1}$ is the Newton constant of the/dual gravity space and $\gamma_{A}$ is the area of the minimal bulk surface whose boundary coincides with the boundary of region $\mathrm{A}$. For a field theory in $1+1$ dimensions, the region A may be taken to be a line of length $\ell$, and the bulk minimal surface $\gamma_{A}$ becomes a bulk geodesic joining the two endpoints of this line. We note that for a 1+1-dimensional CFT at finite temperature, with the BTZ black hole as gravity dual, it is found both in the CFT [112] and on the gravity side [111] that the entanglement entropy for a line of length $\ell$ is given by

$$
S_{\mathrm{BH}}(\ell)=\frac{c}{3} \ln \left(\frac{1}{\pi \epsilon T} \sinh (2 \pi \ell T)\right),
$$

with $\epsilon$ a cut-off parameter.

For the Kondo model, a useful quantity to consider is the impurity entropy which is given by the difference of the entanglement entropies in presence and in absence of the magnetic impurity,

In the previous section we considered the probe limit of the holographic Kondo model, in

$$
S_{\mathrm{imp}}=S_{\text {impurity present }}-S_{\text {impurity absent }} .
$$

which the fields on the $\mathrm{AdS}_{2}$ defect do not backreact on the $\mathrm{AdS}_{3}$ geometry. However, including the backreaction is necessary in order to calculate the effect of the defect on the Ryu-Takayanagi surface. A simple model that achieves this $[113,114]$ consists of cutting the 2+1-dimensional geometry in two halves at the defect at $x=0$ and joining these back together subject to the Israel 


$$
K_{\mu \nu}-\gamma_{\mu \nu} K=-\frac{\kappa_{G}}{2} T_{\mu \nu},
$$

We refer to the joining hypersurface as 'brane'. In (7.8), $\gamma$ and $K$ are the induced metric and extrinsic curvature at the joining hypersurface extending in $(t, z)$ directions. $T_{\mu \nu}$ is the energymomentum tensor for the matter fields $a$ and $\Phi$ at the defect, and $\kappa_{G}$ is the gravitational constant with $\kappa_{G}^{2}=8 \pi G_{N}$. The matter fields $\Phi$ and $a$ lead to a non-zero tension on the brane, which varies with the radial coordinate. The higher the tension on this brane, the longer the geodesic joining the two endpoints of the entangling interval will be. A numerical solution of the Israel junction condition reveals that the brane tension decreases with decreasing temperature, which leads to a shorter geodesic. This in turn leads to a decrease of the impurity entropy (7.7). This decrease is in agreement with the screening of the impurity degrees of freedom.

For large entangling regions $\ell$, we may approximate the impurity entropy to linear order by noting that the length decrease of the Ryu-Takayanagi geodesic $\gamma_{A}$ translates into a decrease of the entangling region $\ell$ itself. To linear order, this implies that the entangling region is given by $\ell+D$ in the UV and by $\ell$ in the IR, for $D \ll \ell$. Using (7.6) we may thus write for the difference of the impurity between its UV and IR values

$$
\begin{aligned}
\Delta S_{\mathrm{imp}} & =S_{\mathrm{BH}}(\ell+D)-S_{\mathrm{BH}}(\ell) \\
& \simeq D \cdot \partial_{\ell} S_{\mathrm{BH}}(\ell)=\frac{2 \pi D T}{3} \operatorname{coth}(2 \pi \ell T) .
\end{aligned}
$$

It is a non-trivial result that subject to identifying the scale $D$ with the Kondo correlation length of condensed matter physics, $D \propto \xi_{K}$, then the result agrees with previous field-theory results for the Kondo impurity entropy [116].

A more extensive review of the holographic Kondo model presented may be found in [117]. Quantum quenches were studied in [118] and correlators and spectral functions in [119,120].

\section{Integrability breaking on the boundary ${ }^{13}$}

The sine-Gordon equation is an initial-value problem for a function $u(x, t)$ in $1+1$ dimensions, obeying the equation of motion

$$
u_{t t}-u_{x x}+\sin (u)=0 .
$$

If space is the full line, $-\infty<x<\infty$, this equation is very well-known to be integrable, with a classical spectrum of kinks, antikinks and breathers. If instead space is cut down to the half-line $-\infty<x \leq 0$, a boundary condition must be given at $x=0$ to make the problem well-posed. A natural question is to ask which of these boundary conditions are compatible with integrability, in the sense of preserving the full set of energy-like conservation laws. Even specialising to the case of no additional boundary degrees of freedom, the full answer was only found surprisingly recently, by Ghoshal and Zamolodchikov in 1994 [70]:

$$
\left.\left[u_{x}+4 K \sin \left(\frac{u-\widehat{u}}{2}\right)\right]\right|_{x=0}=0
$$

where $\hat{u}$ and $K$ are two free parameters. A number of special cases, including (zero) Dirichlet $\left(\left.u\right|_{x=0}=0\right)$ and Neumann $\left(\left.u_{x}\right|_{x=0}=0\right)$, had been known to be integrable before. Ghoshal and Zamolodhikov found their more-general set via a consideration of the lowest-spin extra conserved charges in the full-line model; soon after, in 1995, MacIntyre [71] showed the existence of an infinite set of conservation laws for these boundaries.

Scattering from a Ghoshal-Zamolodchikov boundary is simple: kinks and antikinks reflect perfectly, as either kinks or antikinks, with no loss of energy, and hence no production of radiation or breathers.

${ }^{13}$ This section was authored by Patrick Dorey and Robert Parini. Patrick Dorey is from The Department of Mathematical Sciences, Durham University, Durham, UK. This work is funded in part by STFC consolidated grant number ST/P000371/1. Robert Parini is in the Department of Mathematics, University of York, York, UK. 
However, real life is not integrable, and it might be interesting to explore other, nonintegrable, possibillities, outwith the Ghoshal-Zamolodchikov set. This is a 'minimal' way to break integrability - just at one point - and one could be forgiven for thinking that it wouldn't make much difference. This turns out not to be the case.

In [72] we looked at a natural set of boundary conditions which interpolates between Dirichlet and Neumann in a non-integrable way, namely the one-parameter family of homegeneous Robin boundaries, found by linearising the homogeneous $(\widehat{u}=0)$ cases of the Ghoshal-Zamolodchikov boundary:

$$
\left.\left[u_{x}+2 k u\right]\right|_{x=0}=0
$$

Setting $k=0$ gives Neumann, while $k \rightarrow \infty$ is Dirichlet. Away from these limits, the Robin boundary does not interact nicely with the higher sine-Gordon conserved charges, integrability is lost, and scattering becomes much more complicated. As a first sign of this, figure 6, taken with small modifications from [72], shows the late-time values $u_{\text {late }}$ of the field at $x=0$ for the scattering of an initial sine-Gordon antikink against the Robin boundary, for different initial velocities $v_{0}$ and parameter values $k$.

Figure 6. A 'phase diagram' of late-time field values at $x=0$ : on the left, a full scan; on the right, a zoomed-in view of the small rectangle in the top left quadrant of the full plot.

For the chosen initial condition, the value of the field at $x=-\infty$ is $2 \pi$, and the half-line topological charge of the final configuration is $\tau_{\text {final }}=\left(u_{\text {late }}-2 \pi\right) / 2 \pi$. Roughly speaking, in the red regions, $u_{\text {late }} \approx 4 \pi, \tau_{\text {final }}=1$, and the final configuration contains a kink; in the blue regions, $u_{\text {late }} \approx 0, \tau_{\text {final }}=-1$, and the final configuration contains an antikink; and in the light green regions, $u_{\text {late }} \approx 2 \pi, \tau_{\text {final }}=0$, and the final configuration contains neither kink nor antikink, or else both of them. Looking down the left and right sides of the left-hand plot shows this is consistent with the known behaviours of the two integrable limits: on the left, $k=0$, the boundary condition is Neumann, and an antikink reflects as a kink; while on the right, $k=0.5$, which is near enough to the $k \rightarrow \infty$ (Dirichlet) limit for the initial antikink to be reflected as another antikink. However it is clear that the story is much more complicated in between these two limits. Zooming in to the small rectangular region in the top left corner of the full plot starts to show the extent of 
this complexity: for a given value of the boundary parameter $k$ in this region, a very small change in the initial velocity of the antikink can cause it to be reflected back as an antikink, or not.

At this stage there are two immediate questions: first, how can we disentangle the full soliton content of the final state? So far we only looked at the net topological charge, but this is blind both to breathers and to additional kink-antikink pairs. Second, what's going on? What is the reason for the complicated, almost fractal, structures present in some parts of figure 6 ?

For the first question, we found that the 'direct' part of the full-line inverse scattering method allowed us to make progress. The key idea is that if we wait sufficiently long after the impact of the initial right-moving antikink on the boundary, all excitations will again be far from the boundary, travelling leftwards in a spacial region where integrability still holds. In other words, some sort of 'asymptotic integrability' is at work, whereby integrability is only broken for a finite amount of time. (Unfortunately - or perhaps not, since this is the ultimate origin of the fine fractal-like structures in figure 6 - this finite amount of time can be arbitrarily large, depending on the initial conditions.) Once all excitations have exited stage left from the boundary region something that can be checked numerically by monitoring the amount of energy left near to the boundary - we can patch the numerically-obtained late-time boundary solution onto a full line, and then compute the scattering data for the linear problem associated with the full-line Lax pair to extract the content of kinks, antikinks and breathers. Full details are in [72], while programs implementing our approach can be found at [73].

Concerning the second question, similar 'fractal' structures had been observed a long time ago in a nonintegrable field theory in 1+1 dimensions, namely the $\phi^{4}$ model on the full line (see for example [74-76]). In this model, kinks and antikinks attract each other, and also lose some energy to both radiational and vibrational modes when they scatter. This loss of energy means that below some critical impact velocity $v_{c}$, an incident kink and antikink pair does not have sufficient energy to reseparate after their initial collision, or 'bounce'. However if enough of the lost energy has been 'parked' in a vibrational mode, then on recollision, subject to a suitable resonance condition, it can be returned to the translational modes, allowing the kink and antikink to escape after one or more subsequent bounces, and leading to an elaborate hierarchy of windows of kink-antikink escape, all lying below $v_{c}$. This 'resonant seattering' mechanism has been generalised in various ways, including to the $\phi^{6}$ model where the energy is parked not in the vibrational modes of single kinks and antikinks but rather in the vacuum between a suitably-ordered kink-antikink pair [77], and to the $\phi^{4}$ model on a half-line [78]. However it is not immediately obvious how any of this can apply to the sine-Gordon model, where the kinks and antikinks famously do not have any internal vibrational modes. Nevertheless it turns out that a resonance mechanism is at work in this model, and is the reason behind the intricate structures visible in figure 6. Even though the sine-Gordon kink has no vibrational modes, the breather does oscillate, and in some regimes it is both produced in the initial boundary collision, and also attracted back to the boundary afterwards. This idea was backed up by both numerical and analytical calculations in [72], but there is plenty of room for further work before a full understanding can be claimed.

There are a number of conclusions to be drawn from all of this. We have seen that classical boundary scattering in the sine-Gordon model is surprisingly rich once integrability is broken at the boundary, though many features of the 'phase diagram' of figure 6 remain to be understood. To this end, it would be nice to develop a more effective collective-coordinate description of the boundary situation. This is challenging, as the boundary interaction tends to force the excitation of many other modes, but at least while everything is far from the boundary it is possible that integrability will help. So far in our work, this integrability has only been used in a particularly simple-minded way, just to disentangle the final state after the complicated interactions with the non-integrable boundary have been handled numerically. It is possible that more can be done to exploit integrability in the quarter-plane $\{(x, t): x<0, t>0\}$ which is the domain of our initialvalue problem. The so-called Fokas method (see [79] for a recent review) is one possible avenue for further progress in this regard. Finally, at the back of our minds throughout this work was the thought to look at the corresponding quantum field theory. In the bulk, there has been some

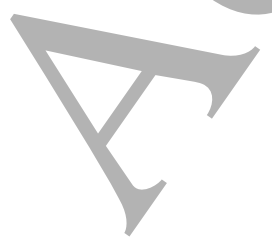


work on the treatment of non-integrable quantum field theories as deformations of integrable theories [80]. In our non-integrable boundary model, there are some features which may allow for additional progress. In particular, the 'asymptotic integrabilty' mentioned above suggests that the space of asymptotic quantum in and out states should be the same as that for integrable boundary scattering, making the model a promising half-way house to a full treatment of integrability breaking in quantum field theory, and one where some of the tools from quantum integrability might be relevant to the study of its breakdown.

\section{Defects in the tricritical Ising Model ${ }^{14}$}

\section{(a) The problem}

The tri-critical Ising model is a minimal model for the Virasoro algebra with central charge $c=$ $7 / 10$. A conformal defect in this model is characterised by an operator $D$ that satisfies

$$
\left(L_{m}-\bar{L}_{-m}\right) D=D\left(L_{m}-\bar{L}_{-m}\right)
$$

The topological defects (which satisfy $L_{m} D=D L_{m}, \bar{L}_{-m} D=D \bar{L}_{-m}$ ) and factorised defects (which satisfy $\left(L_{m}-\bar{L}_{-m}\right) D=D\left(L_{m}-\bar{L}_{-m}\right)=0$ ) have been classified for all Virasoro minimal models [81]; the challenge is to find non-topological non-factorising conformal defects.

There is evidence for the existence of such defects from both perturbation theory and from numerical studies using the truncated conformal space approach but these are not exact results. One way to get exact descriptions is the folding trick: this identifies conformal defects in a CFT with conformal boundary conditions on $\mathrm{CFT}{ }^{\otimes 2}$. The problem is that boundary conditions are only classified for $c<1$ and the central charge of $\operatorname{TCIM}^{\otimes 2}$ is $7 / 5>1$. One way round this is to look for a larger symmetry which simplifies the problem. The tricritical Ising model does not itself have a larger symmetry but it can be constructed from a superconformal minimal model ( $\left.\mathrm{SVIR}_{3}\right)$ with the same central charge. The folded model $\mathrm{SVIR}_{3}{ }^{\otimes 2}$ again has $c=7 / 5$ but this is now a minimal value for the super Virasoro algebra and the folded model is itself a minimal model SVIR 10 . The superconformal boundary conditions for $\mathrm{SVIR}_{10}$ should be classifiable and this could lead to new defects in the tri-critical Ising model.

This idea was proposed by Gang and Yamaguchi in [82]: they proposed boundary states for $\mathrm{SVIR}_{10}$ which would lead to defects in TCIM. There are however two problems. Firstly, the defects predicted by Gang and Yamaguchi do not satisfy the Cardy constraint; secondly, we believe that the defects are not correctly GSO projected and so are not actually defects in TCIM. We have a new proposal, based in part on ideas of Gaiotto for the construction of Renormalisation Group defects. This idea is to use a topological interface $I$ between the TCIM and the NeveuSchwarz sector of $\mathrm{SVIR}_{3}$, and then use the folding trick to obtain defects in $\mathrm{SVIR}_{3}$ as boundary conditions for the Neveu-Schwarz sector of $\mathrm{SVIR}_{10}$. In pictures, we obtain a defect $D^{\prime}$ in $\mathrm{SVIR}_{3}$ from a boundary condition in $\mathrm{SVIR}_{3}{ }^{\otimes 2}=\mathrm{SVIR}_{10}$

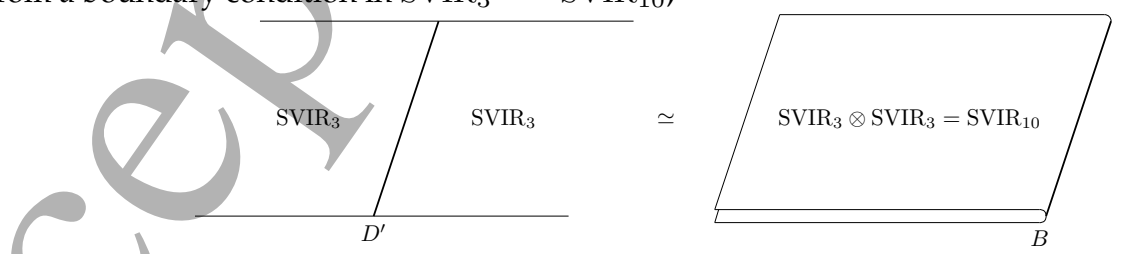

and, from that, obtain a defect in TCIM using topological interfaces: London, The Strand, London WC2R 2LS, UK. This section was based on joint work, published in [83]. 

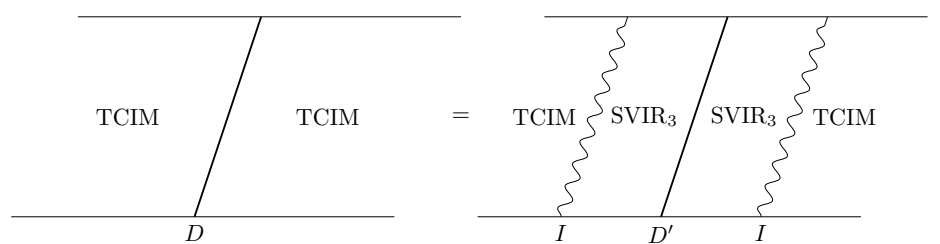

This has the advantage that we can use the Neveu-Schwarz sector of the boundary states found by Gang and Yamaguchi (and do not need the problematic Ramond sector) but has the disadvantage that we cannot expect to produce elementary defects in TCIM.

As a check, we first considered the Ising model (the Virasoro minimal model with $c=1 / 2$ ) which is related to the free fermion in the same way that TCIM is related to SVir. This resulted in four fundamental defects in the free fermion, two topological interfaces between the free fermion and the Ising model, and the reconstruction of a two-dimensional subspace of the known defects in the Ising model.

For SVIR 10 , we found that we could use the Neveu-Schwarz sectors of the boundary states found by Gang and Yamaguchi and, with some changes to choices of representatives (amongst other choices) we could make a uniform presentation of these states. There are 48 boundary states,

$$
\left.\left.\left.\left.\left.\left.\|(a, b)_{N S}\right\rangle\right\rangle, \|(a, b)_{\widetilde{N S}}\right\rangle\right\rangle=(-1)^{F} \|(a, b)_{N S}\right\rangle\right\rangle
$$

They are labelled by a pair of nodes from the Dynkin diagrams of $D_{6}$ and $E_{6}$, bi-coloured as here, and are invariant under $b \mapsto r(b)$, the symmetry of the $E_{6}$ diagram. This would be broken by the addition of Ramond sectors, as originally proposed by Gang and Yamaguchi.

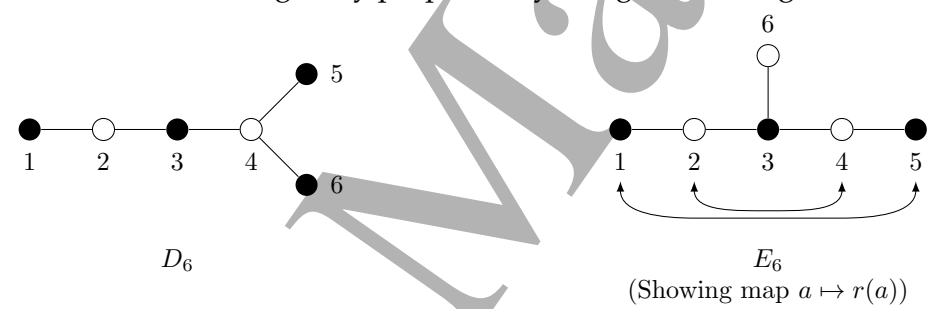

We also found that these split into two distinct sets, depending on whether the colours of the nodes $(a, b)$ are the same or not: we need to use different embeddings of the superconformal algebra for the two sets. Furthermore, we could show that these boundary states are exactly those which preserve a super-W-algebra symmetry, $S W(3 / 2,10)$.

We can calculate easily two properties of the defects: Firstly, the entropy or $g$-value. This decreases along RG flows. The entropy of a superposition of defects is the sum of their energies. Secondly, the transmission coefficient. This takes values $0 \leq T \leq 1$ in a unitary theory. It is 1 for a topological defect and 0 for a factorising defect. A non-topological non-factorising defect will have $0<T<1$.

We found that defects labelled $(a, 1)$ and $(a, 6)$ are either topological or factorising; those labelled $(a, 2)$ and $(a, 3)$ are neither. The defect of lowest entropy is $(1,2)$ with $g=1.015$. and $T=0.633$. This last $g$ value is not the sum of the $g$-values of any combination of known topological or factorising defects.

There was a problem though: the interfaces that convert a defect in $\mathrm{SVIR}_{3}$ into a defect in TCIM are not nicely related to the superconformal structure. This means that we cannot easily find the overlaps of the new defects. Even the overlaps of their constituent Ishibashi states are hard to find. As an example, when the interfaces are included, a typical overlap would be

$$
q^{-\frac{7}{120}}\left[1+\frac{1}{2} q^{\frac{3}{2}}+q^{2}+\frac{1}{2} q^{\frac{5}{2}}+q^{3}+q^{\frac{7}{2}}+\frac{49123}{17689} q^{4}+\frac{3}{2} q^{\frac{9}{2}}+\frac{49123}{17689} q^{5}+\frac{5}{2} q^{\frac{11}{2}}+\frac{102941115}{16999129} q^{6}+\ldots\right]
$$




\section{(b) Conclusions}

We have found convincing evidence for non-topological non-factorising conformal defects in TCIM. We have not been able to find the partition function owing the breaking of superconformal symmetry by the Interface operators. We are currently calculating the perturbative corrections to the transmission coefficients in minimal models to compare with the values found here. We would like to look further into the use of extended algebras to construct new conformal defects. It would be interesting to derive the boundary conditions for the free fermion and $\mathrm{SVIR}_{3}$ from first principles or using the methods of Novak and Runkel [84].

\section{Energy-momentum multiplets for supersymmetric defects and the displacement operator ${ }^{15}$}

\section{(a) Bosonic example}

Let us start with a simple bosonic example, as motivation. We take a $4 \mathrm{~d}$ scalar $\phi$ and a $3 \mathrm{~d}$ scalar $a$, with the latter confined to a planar submanifold $\Sigma$. The $4 \mathrm{~d}$ and $3 \mathrm{~d}$ actions are

$$
\begin{aligned}
\int \mathcal{L}^{(4)} & =\int\left(-\frac{1}{2} \partial^{\mu} \phi \partial_{\mu} \phi-V_{4}(\phi)\right), \\
\int_{\Sigma} \mathcal{L}^{(3)} & =\int_{\Sigma}\left(-\frac{1}{2} \partial^{i} a \partial_{i} a-V_{3}(a)\right) .
\end{aligned}
$$

The energy-momentum tensor includes contributions from both $4 \mathrm{~d}$ and $3 \mathrm{~d}$ fields. and requires the embedding $\delta_{\mu}^{i}$ on the directions tangent to $\Sigma$

$$
T_{\mu \nu}=T_{\mu \nu}^{(4)}+\delta\left(x^{n}\right) \delta_{\mu}^{i} \delta_{\nu}^{j} T_{j i}^{(3)},
$$

where $x^{n}$ is the coordinate normal to $\Sigma$ and

$$
\begin{aligned}
& T_{\mu \nu}^{(4)}=\partial_{\mu} \phi \partial_{\nu} \phi+\eta_{\mu \nu} \mathcal{L}^{(4)} \\
& T_{i j}^{(3)}=\partial_{i} a \partial_{j} a+\eta_{i j} \mathcal{L}^{(3)} .
\end{aligned}
$$

Using the classical equations of motion one finds

$$
\partial^{\mu} T_{\mu \nu}=0
$$

To make the system interesting, we need to couple the $3 \mathrm{~d}$ and $4 \mathrm{~d}$ fields. The simplest way to do that is

$$
\int_{\Sigma} \mathcal{L}^{(I)}=-\int_{\Sigma} V_{I}(\phi, a)
$$

with an arbitrary coupling potential $V_{I}$.

Now the $3 \mathrm{~d}$ term in the energy-moment tensor is

$$
T_{i j}^{(3)}=\partial_{i} a \partial_{j} a+\eta_{i j}\left(\mathcal{L}^{(3)}+\mathcal{L}^{(I)}\right) .
$$

Repeating the calculation, we now find a violation of conservation

$$
\partial^{\mu} T_{\mu \nu}=n_{\nu} \delta\left(x^{n}\right) d, \quad d=\partial_{n} V_{I}(\phi, a) .
$$

\section{(b) The $\mathcal{S}$ multiplet and defects}

Any $4 \mathrm{~d}$ theory with $\mathcal{N}=1$ supersymmetry has an $\mathcal{S}$-multiplet, containing the energy momentum tensor [85]. In the presence of a defect we propose a modification to its equation by the addition

${ }^{15}$ This section was authored by Nadav Drukker from the Department of Mathematics, King's College London, The Strand, London WC2R 2LS, UK. This section was based on work with D. Martelli and I. Shamir [86]. 
of the last term as

$$
\bar{D}^{\dot{\alpha}} \mathcal{S}_{\alpha \dot{\alpha}}=2\left(\chi_{\alpha}-D_{\alpha} X\right)+\delta\left(\tilde{y}^{n}\right) \mathcal{Z}_{\alpha}, \quad \mathcal{Z}_{\alpha}=\Sigma_{\alpha}+\tilde{\Theta}_{\alpha} \Sigma+\left(\Gamma^{i} \tilde{\Theta}\right)_{\alpha} \Sigma_{i}
$$

Here $\mathcal{S}_{\alpha \dot{\alpha}}$ is a real vector superfield, $X$ and $\chi_{\alpha}$ are chiral superfields and $D^{\alpha} \chi_{\alpha}=\bar{D}_{\dot{\alpha}} \bar{\chi}^{\dot{\alpha}}$. The coordinates in the direction normal to the defect are $\tilde{y}^{n}=x^{n}-2 i \tilde{\Theta} \Theta-2 \tilde{\Theta}^{2}$ and $\tilde{\Theta}_{A}=\frac{1}{2}\left(\lambda_{A}^{\alpha} \theta_{\alpha}+\right.$ $\left.\bar{\lambda}_{A}^{\dot{\alpha}} \bar{\theta}_{\dot{\alpha}}\right)$. Finally $\Gamma$ is a $3 \mathrm{~d}$ gamma matrix.

In the $\tilde{\Theta}$ expansion of $\mathcal{S}$ one finds at the linear level

$$
\left.\tilde{\Delta}_{A} \mathcal{S}_{\mu}\right|_{\tilde{\Theta}=0}=-\left(\lambda_{A}^{\alpha} S_{\alpha \mu}+\bar{\lambda}_{A}^{\dot{\alpha}} \bar{S}_{\mu \dot{\alpha}}\right)-4 i\left(\Gamma^{j} \Theta\right)_{A} T_{j \mu}+\ldots
$$

with $S$ and $T$ the supercurrent and energy-momentum tensor, respectively. The components appearing here are those that should be preserved even in the presence of the defect. A straightforward computation then leads to

$$
\left.\delta\left(x^{n}\right)\left(-\frac{i}{4} \Delta^{B} \Delta_{A}\left(\Sigma_{B}+\bar{\Sigma}_{B}\right)-\frac{1}{4}\left(\Gamma^{i} \Delta\right)_{A}\left(\Sigma_{i}+\bar{\Sigma}_{i}\right)\right)\right|_{\tilde{\Theta}=0}+\left.\frac{i}{2} \partial_{n}\left(\delta\left(x^{n}\right)\left(\Sigma_{A}-\bar{\Sigma}_{A}\right)\right)\right|_{\tilde{\Theta}=0} .
$$

So for consistency we should sent the first parenthesis to zero, which imposes conditions on $\Sigma$.

The sub-multiplet of $\mathcal{S}$ containing the violations is given by

$$
\left.\mathcal{S}_{\mu}\right|_{\tilde{\Theta}=0}=-i \Theta^{A}\left(\lambda_{A}^{\alpha} S_{\alpha \mu}-\bar{\lambda}_{A \dot{\alpha}} \bar{S}_{\mu}^{\dot{\alpha}}\right)-2 i \Theta^{2} T_{n \mu}
$$

The derivative is

$$
\left.\partial^{\mu} \mathcal{S}_{\mu}\right|_{\tilde{\Theta}=0}=-\left.\frac{1}{4} \delta\left(x^{n}\right) \Delta^{A}\left(\mathcal{Z}_{A}-\overline{\mathcal{Z}}_{A}\right)\right|_{\tilde{\Theta}=0}-\left.\frac{i}{4} \delta\left(x^{n}\right)\left(\bar{D} \bar{\sigma}_{n} \mathcal{Z}-D \sigma_{n} \overline{\mathcal{Z}}\right)\right|_{\tilde{\Theta}=0} .
$$

Since $T_{n \mu}$ appears in the $\Theta^{2}$ component of $\mathcal{S}_{\mu}$, only the second term contributes to the displacement and is given by the scalar $\Sigma$ piece

$$
\partial^{\mu} T_{n \mu} \sim \delta\left(x^{n}\right) \Delta^{2} \Sigma
$$

like in the bosonic case (10.9). In fact, if we supersymmetrise the above model we will find exactly the same expression (plus the fermion contribution).

\section{Boundary and Defect Conformal Field Theory ${ }^{16}$ \\ (a) The defect set-up}

An interesting 4D defect or boundary conformal field theory can be found within AdS/CFT [8790]. The theory is a special version of $\mathcal{N}=4$ super Yang-Mills theory (SYM) where a co-dimension one defect has been inserted at $x_{3}=0$ and separates two regions of space-time, $x_{3}>0$ and $x_{3}<0$, where the rank of the gauge group is $S U(N)$ and $S U(N-k)$ respectively. The difference in gauge group is implemented by considering $\mathcal{N}=4 \mathrm{SYM}$ with a classical solution where three of the scalar fields are non-vanishing and space-time dependent on one side of the defect, $x_{3}>0$

$$
\phi_{i}^{\mathrm{cl}}=-\frac{1}{x_{3}} t_{i} \oplus 0_{(N-k) \times(N-k)}, \quad i=1,2,3,
$$

where the $t_{i}$ constitute a $k$-dimensional irreducible representation of $S U(2)$. The non-trivial classical solution leads to a partial breaking of conformal as well as super symmetry. The conformal symmetry is reduced from $S O(2,4)$ to $S O(2,3)$ and the R-symmetry is reduced from $S O(6)$ to $S O(3) \times S O(3)$. The full symmetry group of the dCFT is $O S p(4 \mid 4)$.

The above dCFT has a dual string theory description where a probe D5-brane has been embedded in the usual $A d S_{5} \times S^{5}$ background, the embedding being such that the D5-brane geometry is $A d S_{4} \times S^{2}$ and there is a background gauge field with $k$ units of magnetic flux through the $S^{2}$.

${ }^{16}$ This section was authored by Charlotte Kristjansen from Niels Bohr Institute at Copenhagen University, DK-2100 Copenhagen $\varnothing$, Denmark. This work was supported by the Sapere Aude Top Researcher program of the DFF-FNU through grant number DFF-4002-00037 


\section{(b) Integrable one-point functions}

Defect conformal field theories exhibit novel features compared to conformal field theories without defects, one of these being the possibility of non-trivial one-point functions. Symmetries constrain the one-point functions to be of the form

$$
\left\langle\mathcal{O}_{\Delta}(x)\right\rangle=\frac{C}{x_{3}^{\Delta}},
$$

where $\Delta$ is the conformal dimension. One-point functions of the above described defect version of $\mathcal{N}=4$ SYM show very strong signs of integrability. Making use of a boundary state in the form of a specific matrix product state (MPS) and invoking the tools of integrable spin chains one can derive a closed expression for the one-point functions at tree level in the full scalar sector. As is well-known, conformal operators built from only two complex fields, which we will choose as $\phi_{1}+i \phi_{4}$ and $\phi_{2}+i \phi_{5}$, can be identified with the eigenstates $|\mathbf{u}\rangle$ of the Heisenberg spin chain. Accordingly, one can express the corresponding one-point functions (up to a trivial field theoretical pre-factor) as [91]

$$
C_{k}=\frac{\langle\mathrm{MPS} \mid \mathbf{u}\rangle}{\sqrt{\langle\mathbf{u} \mid \mathbf{u}\rangle}}, \quad|\mathrm{MPS}\rangle=\operatorname{tr} \prod_{n=1}^{L}\left[t_{1} \otimes|\uparrow\rangle_{n}+t_{2} \otimes|\downarrow\rangle_{n}\right],
$$

where $k$ refers to the dimension of the representation for the $t_{i}$ and $L$ to the length of the operator (spin chain state). One then finds that the one-point function for $k>2$ can be expressed as

$$
C_{k}=i^{L} T_{k-1}(0) \sqrt{\frac{Q\left(\frac{i}{2}\right) Q(0)}{Q^{2}\left(\frac{i k}{2}\right)}} \sqrt{\frac{\operatorname{det} G_{+}}{\operatorname{det} G_{-}}}
$$

where $T_{k-1}$ is the transfer matrix of the Heisenberg spin chain in the $k$-dimensional representation, $Q(u)$ is the Baxter polynomial, and the matrices $G_{ \pm}$are related to the Gaudin norm of the Bethe state in question as $\langle\mathbf{u} \mid \mathbf{u}\rangle \propto \operatorname{det} G_{+} \operatorname{det} G_{-}$. For $k=2$ one can show that the matrix product state is cohomologically equivalent to a raised version of the Néel state [91,92] and the result for $C_{2}$ can be read off from [93]. The result (11.4) for general $k$ can then be proven by recursion [92]. The formulas above can be extended to the $S U(3)$ sector [94] as well as to the full scalar $S O(6)$ sector [95]. Furthermore, in the case of the $S U(2)$ sector, one can likewise find a closed expression for the one-loop contribution to the one-point function [96]. Finally, based on the observed integrability structure at tree level and one-loop one can make an educated guess for an all loop asymptotic formula for the one-point function of a certain chiral primary, a formula which turns out to agree up to wrapping order with the result of a supergravity calculation carried out in a certain double scaling limit [96], cf. section (c). Overlaps of the type above between a spin chain eigenstate and an initial state in the form of a matrix product state are of relevance for the study of quantum quenches. Recently a possible characterisation of integrable initial states was given in [97].

\section{(c) A quantum check of AdS/dCFT}

Whereas the usual AdS/CFT system is described in terms of only two parameters, the 't Hooft coupling, $\lambda$, and the rank of the gauge group, $N$, one has in the defect set-up an extra tunable parameter, $k$. This fact makes it possible to impose on top of the usual planar limit, $N \rightarrow \infty$, a certain double scaling limit [98]

$$
\lambda \rightarrow \infty, \quad k \rightarrow \infty, \quad \frac{\lambda}{k^{2}} \text { fixed. }
$$

On the string theory side, considering $\lambda \rightarrow \infty$ makes possible a supergravity approximation in which it turns out that the expectation values of certain simple observables organise into an expansion in positive powers the double scaling parameter $\lambda / k^{2}$. Hence, these expectation values can be compared to the result of a standard perturbative field theoretical computation thus 
making possible a comparison of string and field theory in a situation where both supersymmetry and conformal symmetry are partially broken. A comparison based on tree-level results in the field theory gives a match to leading order in the double scaling parameter [99] but the string theory computation also gives rise to predictions about higher loop field theory. For instance, in the case of the chiral primary operator $\mathcal{O}_{L}=\operatorname{Tr}\left(\phi_{1}+i \phi_{4}\right)^{L}$ string theory predicts

$$
\frac{\left\langle\mathcal{O}_{L}\right\rangle_{1-\text { loop }}}{\left\langle\mathcal{O}_{L}\right\rangle_{\text {tree-level }}}=\frac{\lambda}{4 \pi^{2} k^{2}} \frac{L(L+1)}{L-1} .
$$

Setting up the program for perturbative computations in the defect CFT is quite non-trivial due to the classical fields. This task was carried out in $[100,101]$. Expanding around the classical fields one gets not only new cubic interaction terms but also quadratic terms which mix both colour and flavour of the $\mathcal{N}=4$ SYM fields and which in addition carry $x_{3}$-dependence. The diagonalisation of the quadratic terms can be dealt with using fuzzy spherical harmonics, except for a few terms which can be eliminated through gauge-fixing. The resulting masses are still $x_{3}-$ dependent but this dependence can be dealt with by considering the corresponding propagators as propagators in an auxiliary $A d S_{4}$ space. This $A d S_{4}$-space has the defect as its boundary and $x_{3}$ as its radial coordinate. In order to preserve supersymmetry special care is needed in the choice of boundary conditions at the defect for the fields that stay massless. Furthermore, it is crucial that one chooses a supersymmetry preserving regulator when carrying out higher loop computations. When these various precautions are met, one indeed obtains a match with the string theory prediction (11.6) thus achieving a positive quantum check of AdS/CFT in a system where both conformal symmetry and supersymmetry are partially broken $[100,101]$.

\section{(d) Data-mining using defect one-and two-point functions}

Two novel types of two-point functions are possible in the defect set-up namely two-point functions between bulk operators of unequal conformal dimensions and two-point functions involving a bulk field and a defect field. These two-point functions are constrained by symmetries to be of the form

$$
\left\langle\mathcal{O}_{i}(x) \mathcal{O}_{j}(y)\right\rangle=\frac{f_{i j}(\xi)}{\left(x_{3}\right)^{\Delta_{i}}\left(y_{3}\right)^{\Delta_{j}}}, \quad\left\langle\mathcal{O}_{i}(x) \hat{\mathcal{O}}_{\hat{\jmath}}(\vec{y})\right\rangle=\frac{\mu_{i j}}{\left(x_{3}\right)^{\Delta_{i}-\Delta_{j}}|x-(\vec{y}, 0)|^{2 \Delta_{j}}},
$$

where $f_{i j}(\xi)$ is a function of the conformal cross ratio $\xi=\frac{|x-y|^{2}}{4 x_{3} y_{3}}$ and where $\mu_{i j}$ is denoted as a bulk-to-boundary coupling. The bulk two-point functions obey a crossing relation much like fourpoint functions in an ordinary CFT and this relation gives rise to a boundary conformal bootstrap equation $[102,179]$. In one channel the bulk two point function in the presence of the defect can be expressed in terms of one-point functions and three-point functions of the theory without the defect. In the other channel the two-point function can be expressed in terms of one-point functions and bulk-to-boundary couplings. This in particular means that from the knowledge of just one- and two-point functions of the defect set-up one can extract three-point functions of the theory without defect as well as bulk-to-boundary couplings. A number of examples of this type of data-mining was given in [103].

\section{(e) Outlook}

The idea of using one- and two-point functions as input for the boundary conformal bootstrap equations is only in its infancy. Taking it further would potentially be very interesting but in order to fully exploit it for the present dCFT a better understanding of the 3D theory living on the defect is needed. So far the action on the defect has only been explicitly written down for $k=0$ [88]. As regards the integrability properties of one-point functions an interesting question is whether a closed formula can be found even at tree-level for the closely related $S O(5)$ symmetric D3-D7-brane based dCFT. For this field theory one-point functions have been shown to vanish in the $S U(2)$ and $S U(3)$ sub-sectors. One-point functions are non-trivial when the full scalar $S O(6)$

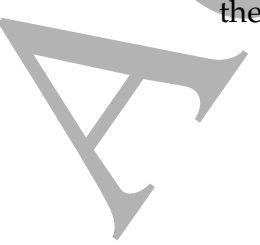




\section{(a) Details}

sector is considered but a closed expression has not yet been found [104] except in the case of chiral primaries [105]. Finally, an open problem is to perform a string theoretical computation of the one-point function of a non-protected operator.

\section{Loop Corrections to Supergravity on $A d S_{5} \times S^{517}$}

The $A d S / C F T$ correspondence relates four-dimensional $\mathcal{N}=4$ Super Yang-Mills (SYM) to type IIB string theory on $A d S_{5} \times S^{5}$ [121-123]. In particular, single-trace chiral primary operators (CPO) of weight $p$ and transforming under the representation $[0, p, 0]$ of the $S U(4) R$-symmetry group, $\mathcal{O}_{p}$, map to supergravity fields with mass $m^{2}=p(p-4)$. Two and three-point correlators of arbitrary CPOs are non renomalized, thus are determined by the free field content of the $\mathcal{N}=$ 4 SYM and do not acquire quantum corrections. Four-point correlators of CPOs are much richer observables, they contain dynamical and generically coupling dependent information. In the limit of large $\lambda=g_{Y M}^{2} N$ four-point correlator of the stress-tensor multiplet (corresponding to $p=2$ ) were computed long ago $[124,125]$. Recently, an elegant algorithm based on symmetries and consistency conditions to determine the four-point correlator of arbitrary CPOs was proposed in $[126,127]$, see also $[128,129]$. In this section we present subleading corrections in $1 / N$ to correlators in the large $\mathrm{t}^{\prime}$ Hooft coupling regime, corresponding to quantum corrections on the gravity side [130].

The analytic bootstrap was initiated in $[131,132]$ and developed into a powerful algebraic machinery in [134-137]. This method allows determining all orders of the large spin expansion of dynamical quantities, such as anomalous dimensions and OPE coefficients, by knowing some specific singularities of the four point correlator. We apply this procedure to compute $\frac{1}{N^{4}}$ corrections to the anomalous dimension of double trace operators of bare dimension $4+\ell$ and spin $\ell$. For the first few values of the spin the results are

The main ingredient is the four point function of $\mathrm{CPOs} \mathrm{O}_{2}$

$$
\left\langle\mathcal{O}_{2}\left(x_{1}\right) \mathcal{O}_{2}\left(x_{2}\right) \mathcal{O}_{2}\left(x_{3}\right) \mathcal{O}_{2}\left(x_{4}\right)\right\rangle=\sum_{\mathcal{R}} \frac{\mathcal{G}^{(\mathcal{R})}(u, v)}{x_{12}^{4} x_{34}^{4}}
$$

where the sum runs over representations in the tensor product $[0,2,0] \times[0,2,0]$ and $u=$ $\frac{x_{12}^{2} x_{34}^{2}}{x_{13}^{2} x_{24}^{2}}, v=\frac{x_{14}^{2} x_{23}^{2}}{x_{13}^{2} x_{24}^{2}}$ are the cross-ratios. Superconformal symmetry allows writing all contributions $\mathcal{G}^{(\mathcal{R})}(u, v)$ in terms of a single non-protected function $\mathcal{G}(u, v)$ satisfying the following crossing relation

$$
v^{2} \mathcal{G}(u, v)-u^{2} \mathcal{G}(v, u)+4\left(u^{2}-v^{2}\right)+\frac{4(u-v)}{c}=0
$$

where $c=\frac{N^{2}-1}{4}$ is the central charge, see [138,139] for a detailed discussion. It is possible to write $\mathcal{G}(u, v)=\mathcal{H}(u, v)+\mathcal{G}^{\text {short }}(u, v)$ in such a way to disentangle the contribution from operators belonging to long and semishort- short multiplets (1/4 and $1 / 2$ BPS operators), respectively. Since both the dimensions and OPE coefficients of the latter are non renormalizable,

${ }^{17}$ This section was authored by Agnese Bissi from the Department of Physics and Astronomy at Uppsala University, Box 516, SE-75120 Uppsala, Sweden. This work was supported in part by the Knut and Alice Wallenberg Foundation under grant KAW 2016.0129. 
the function $\mathcal{G}^{\text {short }}(u, v)$ is completely fixed and known in closed form. Instead $\mathcal{H}(u, v)$ admits a decomposition in superconformal blocks

$$
\mathcal{H}(u, v)=\sum_{\tau, \ell} a_{\tau, \ell} u^{\tau / 2} g_{\tau+4, \ell}(u, v)
$$

where the sum runs over superconformal primary operators in long multiplets, which are singlet of $S U(4)$, with twist (dimension minus the spin) $\tau$ and even spin $\ell$, and $a_{\tau, \ell}$ denotes the squared OPE coefficients. In the limit of infinite central charge $\mathcal{H}(u, v)$ reduces to the generalised free fields result $\mathcal{H}^{(0)}(u, v)$, which agrees with the large $c$ result in the Born approximation (free theory). The intermediate operators correspond to double-trace operators of twist $\tau_{n}=4+2 n$ and the OPE coefficients

$$
a_{n, \ell}^{(0)}=\frac{\pi(\ell+1)(\ell+2 n+6) \Gamma(n+3) \Gamma(\ell+n+4)}{2^{2 \ell+4 n+9} \Gamma\left(n+\frac{5}{2}\right) \Gamma\left(\ell+n+\frac{7}{2}\right)}
$$

The four-point correlator, as well as the twist and the OPE coefficient, can be expanded for large central charge $c$,

$$
\begin{aligned}
& \tau_{n, \ell}=4+2 n+\frac{1}{c} \gamma_{n, \ell}^{(1)}+\frac{1}{c^{2}} \gamma_{n, \ell}^{(2)}+\cdots \\
& a_{n, \ell}=a_{n, \ell}^{(0)}+\frac{1}{c} a_{n, \ell}^{(1)}+\frac{1}{c^{2}} a_{n, \ell}^{(2)}+\cdots
\end{aligned}
$$

At order $1 / c$ and in the limit of large $\lambda$, there is no new operators appearing in the OPE and $\mathcal{H}^{(1)}(u, v)$ can be computed from the classical supergravity result $[126,128]$, with correction to the spectrum and OPE coefficients [133,140,141]

$$
\begin{aligned}
& \gamma_{n, \ell}^{(1)}=-\frac{\kappa_{n}}{(1+\ell)(6+\ell+2 n)}, \\
& a_{n, \ell}^{(1)}=\frac{1}{2} \partial_{n}\left(a_{n, \ell}^{(0)} \gamma_{n, \ell}^{(1)}\right)
\end{aligned}
$$

where $\kappa_{n}=(n+1)(n+2)(n+3)(n+4)$. For a given $n$ and $\ell$ there is more than one superconformal primary in the singlet of $S U(4)$, except for $n=0$. The above corrections should then be interpreted as (weighted-)averages. At order $1 / c^{2}$ since $\mathcal{G}^{\text {short }}(u, v)$ receives contributions only up to order $1 / c$, the crossing equation for $\mathcal{H}^{(2)}(u, v)$ reads

$$
v^{2} \mathcal{H}^{(2)}(u, v)=u^{2} \mathcal{H}^{(2)}(v, u)
$$

We will follow the strategy in [142]: determine the piece proportional to $\log ^{2} u$ in $\mathcal{H}^{(2)}(u, v)$ from the CFT-data at order $1 / c$. By crossing symmetry this leads to a precise divergence proportional to $\log ^{2} v$. Matching this divergence fixes $\gamma_{n, \ell}^{(2)}$ and $a_{n, \ell}^{(2)}$ to all orders in $1 / \ell$. Plugging (12.4) into the conformal block decomposition and expanding up to order $1 / c^{2}$, we find that the piece proportional to $\log ^{2} u$ is

$$
\left.\mathcal{H}^{(2)}(u, v)\right|_{\log ^{2} u}=\sum_{n, \ell} \frac{1}{8} a_{n, \ell}^{(0)}\left(\gamma_{n, \ell}^{(1)}\right)^{2} u^{2+n} g_{n, \ell}(u, v) .
$$

where $g_{n, \ell}(u, v) \equiv g_{\tau_{n}^{(0)}+4, \ell}(u, v)$. An obstacle to compute this is the mixing among double-trace operators $\left[\mathcal{O}_{2}, \mathcal{O}_{2}\right]_{n, \ell},\left[\mathcal{O}_{3}, \mathcal{O}_{3}\right]_{n-1, \ell}, \ldots$ They have the same twist and spin at zeroth order and transform under the same representation of $S U(4)$, hence quantities above should be interpreted as averages, weighted by their respective OPE coefficient at zeroth order. Therefore, the weighted average $\left\langle\left(\gamma_{n, \ell}^{(1)}\right)^{2}\right\rangle$ does not follow from the leading order result, except for $n=0$, for which there is a unique state. This problem can be solved by considering the complete family of four-point correlators $\left\langle\mathcal{O}_{p} \mathcal{O}_{p} \mathcal{O}_{q} \mathcal{O}_{q}\right\rangle$ in generalised free field theory and in the supergravity approximation. 
This allows computing $\left\langle\left(\gamma_{n, \ell}^{(1)}\right)^{2}\right\rangle$ which is given by

$$
\left\langle\left(\gamma_{n, \ell}^{(1)}\right)^{2}\right\rangle=\sum_{p=2}^{\infty} \frac{\alpha_{p} \kappa_{n}^{2}}{\left(J^{2}-(n+2)(n+3)\right)^{2}} \prod_{k=2}^{p-1} \frac{(n-k+2)(n+k+3)}{\left(J^{2}-k(k+1)\right)}
$$

where $\alpha_{p}=p^{2}\left(p^{2}-1\right) / 12$ and $J^{2}=(\ell+n+3)(\ell+n+4)$. Each term inside the sum represents the contribution from the $p$-th $\mathrm{KK}$ mode, or more precisely the intermediate double-trace operators $\left[\mathcal{O}_{p}, \mathcal{O}_{p}\right]$. Crossing symmetry relates this term to a specific combination involving $\gamma_{n, \ell}^{(2)}$ More precisely, it is possible to compute all the terms in the large spin expansion of $\gamma_{0, \ell}^{(2)}$ and to resum them ${ }^{18}$. The resulting contribution for each KK-mode is $\gamma_{n, \ell}^{(2)}-\left.\frac{1}{2} \gamma_{n, \ell}^{(1)} \partial_{n} \gamma_{n, \ell}^{(1)}\right|_{n=0, p}=\alpha_{p} \frac{P^{(14+2 \ell)}(p)}{\left(p^{2}-4\right)\left(p^{2}-1\right) p}+\alpha_{p}\left(p^{2}-4\right)\left(p^{2}-1\right) p^{3} Q^{(4+2 \ell)}(p) \psi^{(2)}(p)$ for some polynomials $P, Q$. At large $p$ we find

$$
\left.\hat{\gamma}_{0, \ell}^{(2)}\right|_{p} \sim \frac{\alpha_{p}}{p^{3+2 \ell}}
$$

since $\alpha_{p} \sim p^{4}$, this implies the sum over $p$ is actually divergent for spin zero. This agrees with the presence of a quadratic divergence in the 10d supergravity computation [143], see a detailed discussion in [144]. For spin two and higher we get a convergent sum which leads to the results quoted in the introduction.

\section{(b) Conclusion}

We have reported results for the CFT-data of unprotected operators in $\mathcal{N}=4$ SYM to order $1 / N^{4}$ and at large 't Hooft coupling. These results appeared in [130], while related discussions are contained in [144-147]. There are open questions that would be nice to address. It would be interesting to discuss $1 / \lambda$ corrections. At leading order this would amount of studying $1 / \lambda$ corrections to the correlators $\left\langle\mathcal{O}_{p} \mathcal{O}_{p} \mathcal{O}_{q} \mathcal{O}_{q}\right\rangle$ which correspond to the addition of the first truncated solution with arbitrary coefficients, which can be fixed in principle along the line of [148]. At order $1 / N^{4}$ one would have to 'square' the supergravity contribution plus these contributions. One could also consider the exchange of a finite number of single-trace operators, combining the results of [149] with the methods of this section. Other interesting directions would be to combine the method presented in this section with recent direct computations of Witten diagrams at one loop [150] and with integrability based approaches [151].

\section{BCFT: Anomalies, Entanglement, Holography ${ }^{19}$}

\section{(a) Introduction}

Physical boundaries are known to result in observable quantum effects, such as Casimir forces between conducting surfaces, for example. Studying and understanding boundary effects, in general, may be a complicated problem which should take into account properties of real materials, interactions and etc. Conformal fields theories (CFTs) with idealized boundaries and boundary conditions (BC) are a useful test bed to develop ones intuition about quantum physics near boundaries. This article is related to recent studies of integrated conformal anomalies (ICA) in CFT's with boundaries (BCFT). ICA are completely determined by a set of bulk and boundary charges. We show that the same charges also fix other important observables in BCFTs near (or on) the boundary. Examples we briefly consider include quantum stress-energy tensor (Casimir energy density), correlations functions of the displacement operator, the entanglement entropy. The entanglement entropy, when the entangling surface crosses the boundary, is a

${ }^{18}$ Results for $n>0$ are discussed in [144]

${ }^{19}$ This section was authored by Dmitri Fursaev from Dubna State University, Universitetskaya str. 19141 980, Dubna, Russian Federation. 
natural quantity to probe the strength of quantum correlations on the boundaries. Interestingly, all above examples allow a holographic description in terms of AdS gravity, at least in a bottom-up approach. For the sake of brevity we consider 4D BCFT's. In odd dimensions ICA consist of pure boundary terms. However analysis of 3D BCFT shows that boundary charges in ICA similarly determine physical observables.

\section{(b) Integrated conformal anomaly in 4D BCFT}

Consider a $4 \mathrm{D}$ BCFT on a background manifold $\mathcal{M}$ with a boundary $\partial \mathcal{M}$. The integral conformal anomaly, ICA, is defined as the variation of the effective action $W$,

$$
\mathcal{A} \equiv \partial_{\sigma} W\left[e^{2 \sigma} g_{\mu \nu}\right]_{\sigma=0}
$$

under scaling with a constant factor $\sigma$. If imposed BC do not break the conformal invariance, ICA has the following universal structure [152-154] :

$$
\mathcal{A}=-2 a \chi_{4}-c i+q_{1} j_{1}+q_{2} j_{2}
$$

Quantities $\chi_{4}, i, j_{1}$ and $j_{2}$ are scale invariant functionals, $\chi_{4}$ is the Euler characteristics of $\mathcal{M}, i$ is the integral over $\mathcal{M}$ of a square of the Weyl tensor $C_{\mu \nu \lambda \rho}$ of $\mathcal{M}, a$ and $c$ are bulk charges. The boundaries result in 2 terms with boundary charges $q_{1}$ and $q_{2}$,

$$
j_{1}=\frac{1}{16 \pi^{2}} \int_{\partial \mathcal{M}} \sqrt{H} d^{3} x C_{\mu \nu \lambda \rho} N^{\nu} N^{\rho} \hat{K}^{\mu \lambda}, j_{2}=\frac{1}{16 \pi^{2}} \int_{\partial \mathcal{M}} \sqrt{H} d^{3} x \operatorname{Tr}\left(\hat{K}^{3}\right) .
$$

Here $N^{\nu}$ is a unit normal vector to $\partial \mathcal{M}$, and $\hat{K}^{\mu \lambda}$ is the traceless part of extrinsic curvature of $\partial \mathcal{M}$.

Let us dwell on some general properties of the charges. Bulk charges $a$ and $c$ are well known for free CFT's. Recently ICA on squashed spheres were used to find these charges for higher spin theories [155]. As was suggested in [156] and proved in [157] $a$-charge monotonically decreases from UV to IR. Actual values for boundary charges are listed in [154] for a number of free BCFT (spins $s=0,1 / 2,1$ ) with Dirichlet or Robin BC. For these models $q_{1}$ do not depend on BC, and are related to $c$-charge: $q_{1}=8 c$. The origin and implications of this relation were discussed in $[3,158]$. It was argued in [3] that the relation may receive quantum corrections due to interactions, at least in certain models. Charges $q_{2}$ depend on BC, except for spin $s=1$.

It is important that $c$-charge is connected with coefficient $C_{T}$ in two-point correlation functions of the stress-energy tensor [159],

$$
\left\langle T_{\mu \nu}(x) T_{\lambda \rho}(0)\right\rangle \sim \frac{C_{T}}{x^{8}} I_{\mu \nu, \lambda \rho}(x) .
$$

One can show that $c=\left(\pi^{2} / 40\right) C_{T}$. Analogously, one can consider correlation functions of the displacement operator $D^{n}$ (which generates variation of the action under a shift of the boundary). The 2-point correlator on $\partial \mathcal{M}$ has the structure:

$$
\left\langle D^{n}(\mathbf{x}) D^{n}(0)\right\rangle \sim \frac{c_{n n}}{\left(\mathbf{x}^{2}\right)^{4}}
$$

where $\mathbf{x}$ are coordinates on $\partial \mathcal{M}$. It was shown in [3] that $q_{1}=4 / 3 c_{n n}$. Remarkably, $q_{2}$ yields a coefficient at a 3-point correlation function of the displacement operator, see [4].

\section{(c) Quantum energy density}

Another set of observables related to ICA is the local energy density near the boundary. It was known long time ago [160] that the renormalized expectation value of the stress-energy tensor near the boundary allows an asymptotic expansion in terms of inverse powers of the geodesic distance from a point to the boundary. Recently it was shown [161] that for free 4D BCFT $(s=0,1)$ 
this expansion is fixed by boundary charges:

$$
\begin{gathered}
\left\langle T_{\mu \nu}(\mathbf{x}, y)\right\rangle \sim \frac{1}{16 \pi^{2}}\left(\frac{q_{1}}{y^{3}} \hat{K}_{\mu \nu}(\mathbf{x})+\frac{t_{\mu \nu}(\mathbf{x})}{y^{2}}+\ldots\right) \\
t_{\mu \nu}(\mathbf{x})=\frac{1}{3} q_{1}\left(-2 N_{(\mu} H_{\nu)}^{\lambda}\left(\partial_{\lambda} K+R_{\lambda \alpha} N^{\alpha}\right)+\frac{1}{6} q_{1} K \hat{K}_{\mu \nu}-\right. \\
\left(2 q_{1}+3 q_{2}\right)\left(\hat{K}_{\mu \beta} \hat{K}_{\nu}^{\beta}-\frac{1}{3} H_{\mu \nu} \hat{K}_{\alpha \beta} \hat{K}^{\alpha \beta}\right)
\end{gathered}
$$

Here we use geodesic coordinates, where a point on $\mathcal{M}$ has coordinates $(y, \mathbf{x}), y$ being a geodesic distance from it to a point on $\partial \mathcal{M}$. Curvatures in (13.7) are calculated at $\partial \mathcal{M}$.

\section{(d) Entanglement entropy}

Consider a BCFT specified by a density matrix $\hat{\rho}$. Entanglement entropy between states located in spatially separated parts, $A$ and $B$, with a common boundary $\mathcal{B}$ is determined by a reduced density matrix (for the region $A$ ) $\hat{\rho}_{A}=\operatorname{Tr}_{B} \hat{\rho}$. The entanglement Rényi entropy of an order $q$ is defined as $S_{q}(\mathcal{B})=\ln \operatorname{Tr}_{A} \hat{\rho}_{A}^{q} /(1-q)$, where $q$ is a non-negative parameter, $q \neq 1$. The corresponding entanglement entropy $S(\mathcal{B})=-\operatorname{Tr}_{A} \hat{\rho}_{A} \ln \hat{\rho}_{A}$ follows from $S_{q}(\mathcal{B})$ in the limit $q \rightarrow 1$. One can consider integer values $q=2,3, \ldots$, then $S_{q}=\left(q W-W_{q}\right) /(1-q)$, where $W_{q}$ is the effective action of the theory on a manifold $\mathcal{M}_{q}, W_{1}=W . \mathcal{M}_{q}$ is constructed from $q$ copies of the initial background space $\mathcal{M}$ and has conical singularities at $\mathcal{B}$. Correspondingly, $S=$ $\partial_{q=1} W_{q}-W$. The entanglement entropy is a divergent quantity,

$$
S(\mathcal{B}) \simeq \frac{A(\mathcal{B})}{\varepsilon^{2}}+\frac{P(\mathcal{B})}{\varepsilon^{2}}+s_{\log } \ln \varepsilon+\cdots,
$$

where $\varepsilon$ is a UV cutoff, $A(\mathcal{B})$ and $P(\mathcal{B})$ are the area and perimeter of $\mathcal{B}$, respectively (we omit numerical prefactors in the first two terms). The perimeter term appears when $\mathcal{B}$ crosses $\partial \mathcal{M}$ [162]. This is the case we are interested in. Let $\mathcal{A}_{q}$ be ICA for $W_{q}$. The ICA determines logarithmic divergences of the entropy, $s_{\log }=\partial_{q=1} \mathcal{A}_{q}-\mathcal{A}$. By using this one can show that [163]

$$
s_{l o g}=a F_{a}+c F_{c}+b F_{b}+d F_{d}+e F_{e}
$$

Here $F_{a}, F_{c}, F_{b}$ are some conformal invariants on $\mathcal{B}$ which depend on internal geometry of $\mathcal{M}$, topology and external geometry of $\mathcal{B} . F_{d}$ and $F_{e}$ are some other set of conformal invariants on $\mathcal{C}=\partial \mathcal{M} \cap \mathcal{B}$ which appear only when $\mathcal{B}$ crosses $\partial \mathcal{M}$. One can show that $b=c$, see [164] and references therein. Therefore, bulk charges $a, c$ fix uniquely the logarithmic divergences of the entropy in the absence of boundaries.

$F_{d}$ is a conformally invariant integral on $\mathcal{C}$ of a projection, $\hat{K}_{v v}$, of components of the extrinsic curvature of $\partial \mathcal{M}$ on a tangent vector to $\mathcal{C}$. Another invariant $F_{e}$ depends on extrinsic curvatures of $\mathcal{B} . F_{d}$ and $F_{e}$ can be defined up to conformally invariant dimensionless prefactors depending on a tilt angle between $\mathcal{B}$ and $\partial \mathcal{M}$. Fortunately, when $\mathcal{B}$ is orthogonal to $\partial \mathcal{M}, F_{e}$ vanishes and $F_{d}$ is unique. So one can fix $d$ for a set of free BCFT $[163,165]$. Remarkably, $d$-charges appear to be related to ICA as $d=3 a-14 c-35 / 12 q_{2}$, see [165], thus ICA determines $s_{\text {log }}$ in BCFT.

\section{(e) Holography}

A first attempt to develop a holographic approach to BCFT was done in [166]. The basic idea of [166] is that a holographic dual of a BCFT is an AdS gravity one dimension higher in a domain restricted by a hypersurface $\mathcal{S}(\mathcal{S}$ being dual to $\partial \mathcal{M}) . \mathcal{S}$ is a dynamical surface whose equations depend on boundary conditions. Two types of equations were suggested for $\mathcal{S}$ in [167] and in [168] with the aim to reproduce ICA in supersymmetric BCFT. For instance, $\mathcal{S}$ was treated in [168] as a minimal surface. This allowed the authors to derive holographically the boundary charges in ICA for $\mathcal{N}=4$ Super $S U(N)$ YM theory (assuming that the charges are protected from quantum corrections by the supersymmetry). BC were chosen to break half of supersymmeties . 
Holographic Ryu-Takayanagi formula [169] reproduces successfully the logarithmic part, see (13.9), of entanglement entropy [170] in the absence of boundaries. The holographic formula was used in [165] for of BCFT's (the Super $S U(N)$ YM) by using prescription of [168] and reproduced (13.9) in case when $\mathcal{B}$ is orthogonal to the boundary.

\section{Blocks for Conformal Defects ${ }^{20}$}

\section{(a) Introduction}

Extended objects such as line or surface operators, defects, interfaces and boundaries áre important probes of the dynamics in quantum field theory. They give rise to observables that can detect a wide range of phenomena including phase transitions and non-perturbative dualities. In 2-dimensional conformal field theories, they also turned out to play a vital role for modern formulations of the bootstrap programme. In fact, in the presence of extended objects, the usual crossing symmetry becomes part of a much larger system of sewing constraints [6]. While initially the 2-dimensional bootstrap started from the crossing symmetry of bulk four-point functions to gradually bootstrap correlators involving extended objects, better strategies were adopted later which depart from some of the sewing constraints involving extended objects. The usual crossing symmetry constraint is then solved at a later stage to find the bulk spectrum and operator product expansion, see e.g. [171].

The bootstrap programme, whether in its original formulation [172] or in the presence of extended objects, relies on conformal partial wave expansions $[173,174]$ that decompose physical correlation functions into kinematically determined blocks/partial waves and dynamically determined coefficients.

In the original bulk bootstrap program, the conformal blocks for a four-point correlator are functions of two cross ratios and the coefficients are those that appear in the operator product expansion of local fields. Such conformal partial wave expansions thereby separate very neatly the dynamical meat of a conformal field theory from its kinematical bones.

In order to perform a conformal partial wave expansion one needs a good understanding of the relevant conformal blocks. While they are in principle determined by conformal symmetry alone, it is still a highly non-trivial challenge to identify them in the zoo of special functions, determine their analytical properties or to develop efficient expansion formulas. In the case of scalar fourpoint functions much progress had been made in the conformal field theory literature starting with [175-177]. If the dimension $d$ is even, one can actually construct the conformal blocks from products of two hypergeometric functions each of which depends on one of the cross ratios. For more generic dimensions many important properties of the scalar blocks had been understood. These include their detailed analytical structure and various series expansions.

Extended objects give rise to new families of blocks. There are a few cases, such as boundary or defect 2-point functions, which involve only one or two cross ratios. In these cases it turns out that the relevant blocks are made from the same functions as for bulk four-point functions, $[178,179]$. Hence all the knowledge on blocks that had been assembled in the context of bulk four-point functions carries over to the case of two bulk fields in the presence of a boundary or defect. But what about more general situations such as e.g. the correlation function of two (Wilsonor 't Hooft) line operators in a $d$-dimensional conformal field theory? Such configurations often possess more than two conformal invariant cross ratios. Two conformal line operators in a 4dimensional theory, for example, give rise to three cross ratios. For a configuration of a $p$ - and a $q$-dimensional object in a $d$-dimensional theory, the number of cross ratios is given by $N=$ $\min (d-p, q+2)$ if $p \geq q$ [180]. So clearly, the study of such defect correlation functions involves new types of special functions which depend on more than two variables.

${ }^{20}$ This section was authored by Volker Schomerus from the DESY Theory Group at DESY Hamburg, Notkestrasse 85, D-33607 Hamburg, Germany and the Department of Particle Physics and Astrophysics at the Weizmann Institute of Science, Rehovot 76100 , Israel. 
In order to explore the features of these new functions, understand their analytical properties or find useful expansions one could try to follow the same route that was used for scalar fourpoint blocks, see e.g. [181] for some recent work in this direction. It is the central message of this short paper, however, that there is another route that gives much more direct access to defect blocks. It relies on a generalization of an observation in [182] that 4-point blocks are wave functions of certain integrable 2-particle Hamiltonians of Calogero-Sutherland type [183,184]. The solution theory for this quantum mechanics problem is an important subject of modern mathematics, starting with the seminal work of Heckman-Opdam [185], see [186] for a recent review in the context of conformal blocks. Much of the development in mathematics is not restricted to the 2-particle case and it has given rise to an extensive branch of the modern theory of multi-variable hypergeometric functions which are quite well understood.

This proceedings is based on the works [186-188,191].

\section{(b) Main Results}

In order to put all this mathematical knowledge to use in the context of defect blocks, we need to link the corresponding conformal blocks, which depend on $N$ variables, to wave functions of an $N$-particle Calogero-Sutherland model. That such a link exists is the main claim of this work. Following a general route through harmonic analysis on the conformal group [187], one can indeed construct systematically the relevant Calogero-Sutherland Hamiltonian. For all values of the dimensions $d, p, q$ it takes the form

$$
\begin{aligned}
H_{\mathrm{CS}}= & -\sum_{i=1}^{N} \frac{\partial^{2}}{\partial \tau_{i}^{2}}+\frac{k_{3}\left(k_{3}-1\right)}{2} \sum_{i<j}^{N}\left[\sinh ^{-2}\left(\frac{\tau_{i}-\tau_{j}}{2}\right)+\sinh ^{-2}\left(\frac{\tau_{i}+\tau_{j}}{2}\right)\right] \\
& +\sum_{i=1}^{N}\left[k_{2}\left(k_{2}-1\right) \sinh ^{-2}\left(\tau_{i}\right)+\frac{k_{1}\left(2 k_{2}+k_{1}-1\right)}{4} \sinh ^{-2}\left(\frac{\tau_{i}}{2}\right)\right] .
\end{aligned}
$$

The coupling constants $k_{i}, i=1,2,3$ that appear in the potential can assume complex values though we will mostly be interested in cases in which they are real. The coordinates $\tau_{i}$ may also be complex in general as long as the potential remains real.

Our claim is that all Casimir equations for defect blocks can be recast into an eigenvalue equation for the Calogero-Sutherland Hamiltonian (14.1) with coupling constants depending on the choice of defects and the dimension $d$. In the case of two defects of dimension $p \geq q$ with $q \neq 0$, the relevant couplings read

$$
k_{1}=N-\frac{d}{2}, \quad k_{2}=\frac{p-q}{2}, \quad k_{3}=\frac{1}{2} .
$$

The Calogero-Sutherland problem is to be considered on the semi-infinite cuboid that is parametrized by a real coordinate $\tau_{1} \in \mathbb{R}_{+}$and $N-1$ angles $\varphi_{i}, i=2, \ldots, N$, such that $\tau_{i}=i \varphi_{i}$. When interpreted in terms of the geometry of two spherical defects, $\tau_{1}$ parametrizes the ratio of their radii while $\tau_{2}, \ldots, \tau_{N}$ relative rotation angles. Precise formulas will be presented in our forthcoming paper.

The formulas (14.2) still apply when $q=0$, but in this case there exists an important extension. A zero-dimensional defect may also be thought of as the insertion of two local bulk scalar fields. When these possess the same conformal weight, the coupling constants of the associated Calogero-Sutherland model are given by eq. (14.2). If the conformal weight of the two bulk fields differs by $2 a=\Delta_{2}-\Delta_{1} \neq 0$ the coupling constants become

$$
k_{1}=2-\frac{d}{2}, \quad k_{2}=\frac{p}{2}, \quad k_{3}=\frac{1}{2}+a
$$

These results have direct implications on relations between different types of blocks. One such example concerns the case of two bulk fields in the presence of a defect that we have just discussed. Comparison of the Calogero-Sutherland model with couplings (14.3) with that for bulk 
four-point functions [182] shows that the defect blocks are related to the blocks for four scalar fields with conformal weights $\Delta_{i}^{\prime}$ satisfying $2 a=\Delta_{2}^{\prime}-\Delta_{1}^{\prime}$ and $0=\Delta_{3}^{\prime}-\Delta_{4}^{\prime}$. This generalizes an observation in [178]. Several similar relations will be discussed in the forthcoming paper.

Our formulas for the coupling constants may be derived by restricting the Laplace operator on the conformal group manifold to the quotient space $G_{p} \backslash G / G_{q}$ where $G=\operatorname{SO}(1, d+1)$ is the conformal group and $G_{p}=\mathrm{SO}(1, p+1) \times \mathrm{SO}(d-p)$ the subgroup that leaves the defect invariant. It is not difficult to see that the action of $G_{p} \times G_{q}$ on the conformal group $G$ is stabilized by the diagonal subgroup $B_{p q}=\mathrm{SO}(p-q) \times \mathrm{SO}(|d-p-q-2|)$. Once this is taken into account, it is straightforward to compute the dimension of the double coset space,

$$
\operatorname{dim} G_{p} \backslash G / G_{q}=\operatorname{dim} G-\operatorname{dim} G_{p}-\operatorname{dim} G_{q}+\operatorname{dim} B_{p q}=N
$$

Hence, after reduction to the double coset, the Laplace operator on the conformal group becomes a second order operator in $N$ variables. The latter can be transformed into a multiparticle Hamiltonian of Calogero-Sutherland type, see [187-190] for related discussions and our forthcoming paper for a derivation in the case of defect blocks.

\section{(c) Outlook}

Once the connection between defect blocks and Calogero-Sutherland models is established, one can exploit the extensive mathematical theory. In fact, the study of Calogero-Sutherland models became an important subject in mathematics, starting with the work of Heckman and Opdam [185] that initiated much of the modern theory of multi-variable hypergeometric functions. A lightning review of some central results can be found in $[186,191]$. The relevant defect blocks may be constructed explicitly in terms of the well studied class of Harish-Chandra functions. Many profound results such as series expansions, poles and their residues in momentum space, as well as global analytical properties including the position of cuts and their monodromies can be found in the literature or at least be derived from published results.

\section{CFTs on real projective spaces ${ }^{21}$}

Suppose we know everything about a conformal field theory (CFT) in flat Minkowski space-time, how much can we solve the same CFT in non-trivial curved space-time? We have a gut feeling that we should be able to do it because in the Lagrangian formalism, once we know the action in flat space-time, the action in the curved background is more or less fixed (up to some arbitrariness from curvature induced interactions), and in principle we should able to solve it.

In practice, however, it seems very hard to accomplish this, when the theory is strongly coupled with or without the Lagrangian formalism. The aim of this talk is to discuss how much we can solve the CFT on a certain curved background, a real projective space, given the CFT data on the flat Euclidean space.

The real projective space is defined by the identification of anti-podal points on the $d$ dimensional sphere. Let us do the conformal map from the sphere to a plane $R^{d}$, then the anti-podal identification becomes the indentification under the involution $\vec{x} \rightarrow-\frac{\vec{x}}{\vec{x}^{2}}$. We may further conformal transform to a cylinder $R_{t} \times S_{d-1}$, in which the involution becomes a "PT" transformation $(t, \vec{\Omega}) \rightarrow(-t,-\vec{\Omega})$. On the cylnder, one may introduce the crosscap state $|C\rangle$ that satisfies $M_{a b}|C\rangle=\left(P_{a}+K_{a}\right)|C\rangle=0$ at $t=0$ to implement the identification.

Solving the CFT on the real projective space is equivalent to computing one-point functions of all the scalar primary operators $\left\langle O_{i}(\vec{x})\right\rangle=\frac{A_{i}}{\left(1+\vec{x}^{2}\right)_{i}}$ so that we can compute all the correlation functions by repeated use of operator product expansions (OPE) given a flat space CFT data. The

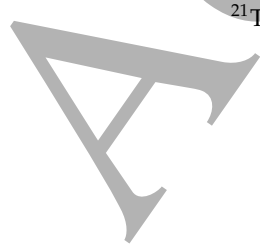

${ }^{21}$ This section was authored by Yu Nakayama from The Department of Physics, Rikkyo University, Tokyo, Japan. 


$$
|C\rangle\rangle=\sum_{i} A_{i} \Gamma\left(\Delta_{i}-d / 2+1\right)\left(\frac{\sqrt{p^{2}}}{2}\right)^{\frac{d}{2}-\Delta_{i}} J_{\Delta_{i}-\frac{d}{2}}\left(\sqrt{p^{2}}\right)\left|O_{i}\right\rangle
$$

where $p$ is the momentum operator.

To test our philosophy of solving CFTs on curved space-time from the given CFT data on the flat space-time, let us try the bootstrap analysis in which we study the consistency of the two-point functions on the real projective space [192]. The scalar two-point function takes the form

$$
\left\langle O_{1}\left(\vec{x}_{1}\right) O_{2}\left(\vec{x}_{2}\right)\right\rangle=\frac{\left(1+\vec{x}_{1}^{2}\right)^{\frac{-\Delta_{1}+\Delta_{2}}{2}}\left(1+\vec{x}_{2}^{2}\right)^{\frac{-\Delta_{2}+\Delta_{1}}{2}}}{\left(\vec{x}_{1}-\vec{x}_{2}\right)^{2\left(\frac{\Delta_{1}+\Delta_{2}}{2}\right)}} G_{12}(\eta)
$$

where $\eta=\frac{\left(\vec{x}_{1}-\vec{x}_{2}\right)^{2}}{\left(1+\vec{x}_{1}^{2}\right)\left(1+\vec{x}_{2}^{2}\right)}$ is so-called crosscap cross ratio. By noticing the identification $O_{i}(\vec{x}) \sim$ $O_{i}\left(-\frac{\vec{x}}{\vec{x}^{2}}\right)$, we demand the crossing symmetry

$$
\left(\frac{1-\eta}{\eta^{2}}\right)^{\frac{\Delta_{1}+\Delta_{2}}{6}} G_{12}(\eta)=\left(\frac{\eta}{(1-\eta)^{2}}\right)^{\frac{\Delta_{1}+\Delta_{2}}{6}} G_{12}(1-\eta)
$$

One may solve the crossing equation exactly in $d=2$ minimal models by using the Virasoro conformal block decomposition of $G(\eta)$.

In $d>2$ dimensions, we use the global conformal block decomposition

$$
G_{12}(\eta)=\sum_{i} C_{12 i} A_{i} \eta^{\Delta_{i} / 2}{ }_{2} F_{1}\left(\frac{\Delta_{1}-\Delta_{2}+\Delta_{i}}{2}, \frac{\Delta_{2}-\Delta_{1}+\Delta_{i}}{2} ; \Delta_{i}+1-\frac{d}{2} ; \eta\right),
$$

but here we may have to take the sum over infinitely many primary operators (except for certain favorable cases like free field theories). In the numerical analysis, we truncate the sum.

Let us focus on the Ising-like theories and study the two-point functions of spin operators with the OPE $[\sigma] \times[\sigma]=1+[\epsilon]+\left[\epsilon^{\prime}\right]+\cdots$. In $d=4-\epsilon$ dimensions, we find the exact (i.e. to all order in $\eta$ expansion) expression for the crossing symmetric two-point function

$$
\begin{aligned}
G(\eta) & =1+(1-a \epsilon) \eta^{1-\frac{\epsilon}{2}+a \epsilon}{ }_{2} F_{1}\left(1-\frac{\epsilon}{2}+a \epsilon, 1-\frac{\epsilon}{2}+a \epsilon, 2-\epsilon+2 a \epsilon+1-\frac{4-\epsilon}{2} ; \eta\right) \\
& +\frac{a \epsilon}{2} \eta^{2-\epsilon}{ }_{2} F_{1}\left(2-\epsilon, 2-\epsilon, 4-2 \epsilon+1-\frac{4-\epsilon}{2} ; \eta\right)+O\left(\epsilon^{2}\right)
\end{aligned}
$$

up to $O\left(\epsilon^{2}\right)$ corrections, where $a$ is arbitrary but is related to $\gamma_{\phi^{2}}=2 a \epsilon$.

Beyond this order, we need an infinite number of primary operators to satisfy the crossing symmetry at $\eta=0$ or $\eta=1$, but the convergence is exponentially fast at $\eta=1 / 2$. In $d=2$ dimensions, the truncated bootstrap gives the numerical prediction

\begin{tabular}{|c||c|c|c|c|c|}
\hline & Exact & 2 & 3 & 4 & 5 \\
\hline \hline$C_{\sigma \sigma \epsilon} A_{\epsilon}$ & 0.20711 & 0.20407 & 0.20757 & 0.20693 & 0.20710 \\
\hline$C_{\sigma \sigma \epsilon^{\prime}} A_{\epsilon^{\prime}}$ & 0.01563 & 0.01702 & 0.01539 & 0.01572 & 0.01563 \\
\hline
\end{tabular}

which is in good agreement with the exact value $C_{\sigma \sigma \epsilon}=\frac{\sqrt{2}-1}{2}=0.20711$. In $d=3$ dimensions, we predict

\begin{tabular}{|c||c|c|c|c|c|}
\hline & $(2,0)$ & $(4,0)_{\mathrm{A}}$ & $(4,0)_{\mathrm{B}}$ & $(6,0)_{\mathrm{B}}$ & $(4,0)_{\mathrm{S}}$ \\
\hline$C_{\sigma \sigma \epsilon} A_{\epsilon}$ & 0.690 & 0.7015 & 0.7022 & 0.70197 & 0.6908 \\
\hline$C_{\sigma \sigma \epsilon^{\prime}} A_{\epsilon^{\prime}}$ & 0.054 & 0.0475 & 0.0470 & 0.04714 & 0.0549 \\
\hline
\end{tabular}

The similar analysis can be done in the critical Lee-Yang model, whose $\epsilon$ expansion is discussed in [193]. In the future, it would be interesting to study holographic models such as $N=4$ super Yang-Mills theory or Liouville theory and compare them with the holographic predictions. 


\section{Conformal field theories with and without boundaries: from a holographic picture to logarithmic CFT 22}

Correlators in rational conformal field theories can be obtained by the TFT construction. This construction, which is summarized e.g. in [194], amounts to the following. Assume we are given a chiral conformal field theory for which the conformal blocks and their monodromy are encoded in a (semisimple) modular tensor category $\mathcal{C}$. Such a category allows for the construction of a three-dimensional topological field theory $\mathrm{tft}_{\mathcal{C}}$ of Reshetihkin-Turaev type. By the principle of holomorphic factorization, a correlator on an (oriented) surface $X$ for these chiral data $\mathcal{C}$ is an element $\operatorname{Cor}_{X} \in \operatorname{tft}_{\mathcal{C}}(\widehat{X})$, where $\widehat{X}$ is the oriented double of $X$. A consistent set of correlators is the datum of such a vector $\operatorname{Cor}_{X} \in \operatorname{tft}_{\mathcal{C}}(\widehat{X})$ for every surface $X$, such that the vector for $X$ is invariant under the action of the mapping class group of $X$ on the vector space $\operatorname{tft}_{\mathcal{C}}(\widehat{X})$, and that the vectors for different $X$ are compatible with the factorization (or sewing) of surfaces.

Such a collection of correlators can be constructed for any special symmetric Frobenius algebra in $\mathcal{C}$ in terms of the topological field theory $\operatorname{tft}_{\mathcal{C}}$ as follows: Given a surface $X$, one constructs a three-manifold $M_{X}$ with boundary $\partial M_{X}=\widehat{X}$ and with an embedded ribbon graph such that $\operatorname{Cor}_{X}=\operatorname{tft}_{\mathcal{C}}\left(\emptyset \stackrel{M_{X}}{\longrightarrow} \widehat{X}\right)(1) \in \operatorname{tft}_{\mathcal{C}}(\widehat{X})$. This constitutes a mathematically precise holographic approach to RCFT correlators which uses a three-dimensional topological field theory in the bulk. In this rigorous framework one can prove that consistent systems of correlators on all surfaces are in bijection with (classes of) special symmetric Frobenius algebras in $\mathcal{C}$, and OPE coefficients as well as coefficients of partition functions can be expressed as invariants of links in three-manifolds.

The Frobenius algebra enters the construction of the correlator for a surface $X$ via a trivalent graph, dual to a triangulation of $X$. This dual triangulation can be seen as the remnant of a surface defect in the three-dimensional topological field theory; see [195] and [196, Sect. 6] for the relation to special symmetric Frobenius algebras.

More recent developments transcend the realm of rational CFT, dealing with logarithmic conformal field theories, whose chiral data are described by a non-semisimple finite modular tensor category $\mathcal{C}$. (Examples of such logarithmic conformal field theories are the minimal models of type $\mathcal{W}_{1, p}$.) For such theories the physical idea of "summing over all intermediate states" can be implemented by the categorical notion of a coend [197]. Combining this idea with the one of a Lego-Teichmüller game in the sense of [198], in which a modular functor is built from three-point conformal blocks on the sphere via sewing, one arrives at a mathematically precise description of the monodromy data of the conformal blocks of such a chiral logarithmic conformal field theory.

A construction of correlators of a logarithmic conformal field theory is achieved by implementing these ideas for the category $\mathcal{C} \otimes \mathcal{C}^{\text {rev }}$ which accounts for the presence of both leftand right-movers. (Here $\mathcal{C}^{\text {rey }}$ is the modular category in which over-braidings are replaced by under-braidings and vice versa.) This way it has been shown [199] that consistent systems of correlators of bulk fields are in bijection with modular Frobenius algebras in $\mathcal{C} \otimes \mathcal{C}^{\text {rev }}$. These are commutative symmetric Frobenius algebras satisfying one additional property that ensures consistency of higher genus correlators. The physical interpretation of the Frobenius algebra is as the algebra of bulk fields.

In the case that $\mathcal{C}$ is the category of finite-dimensional modules over a finite-dimensional factorizable ribbon Hopf algebra $H$ it is known [200] that the coend object

$$
\int^{c \in \mathcal{C}} \omega(c) \otimes c^{\vee} \in \mathcal{C} \otimes \mathcal{C}^{\mathrm{rev}}
$$

has the structure of a modular Frobenius algebra, for any ribbon automorphism $\omega$ of $H$. This can be interpreted as a generalization of modular invariant partition functions of automorphism type to logarithmic conformal field theories.

${ }^{22}$ This section was authored by Christoph Schweigert from Fachbereich Mathematik, Universität Hamburg Bereich Algebra und Zahlentheorie, Bundesstraße 55, 20146 Hamburg, Germany. 
Specifically, for $\omega$ the identity, the result can be regarded as a generalization of the Cardy case to logarithmic conformal field theories. In this case the coefficients of the torus partition function are [201] given by the entries of the Cartan matrix of the category $\mathcal{C}$, i.e. by the dimensions

$$
c_{i j}=\operatorname{dim} \operatorname{Hom}_{\mathcal{C}}\left(P_{i}, P_{j}\right)
$$

of Hom-spaces involving the indecomposable projective objects in $\mathcal{C}$.

\section{A new kind of quantum field theory of (n-1)-dimensional defects in $2 \mathrm{n}$ dimensions ${ }^{23}$}

This is a summary of the main points of [203]. A short summary is [204]. More expositions appear at [205].

Let $M$ be euclidean space-time: an oriented conformal $2 n$-manifold, compact, without boundary. When $n=1, M$ is a Riemann surface. The basic examples are $M=S^{2 n}=\mathbb{R}^{2 n} \cup\{\infty\}$. The Hodge $*$-operator acting on $n$-forms is conformally invariant, with $*^{2}=(-1)^{n}$. Nothing else is used of the conformal structure on $M$.

The physical objects are represented mathematically as the integral $(n-1)$-currents in $M$, as constructed in Geometric Measure Theory [206]. A $k$-current $\xi$ in $M$ is a distribution on the smooth $k$-forms, $\omega \mapsto \int_{\xi} \omega \in \mathbb{R}$. The boundary operator $\partial$ is dual to the exterior derivative, $\int_{\partial \xi} \omega=\int_{\xi} d \omega$, $\partial^{2}=0$. A singular $k$-chain is an integer linear combination $\sigma=\sum_{i} m_{i} \sigma_{i}$ of $k$-simplices $\sigma_{i}: \Delta^{k} \rightarrow$ $M$ in $M$. The singular $k$-currents $\mathcal{D}_{k}^{\text {sing }}(M)$ are the currents $\int_{[\sigma]} \omega=\sum_{i} m_{i} \int_{\Delta^{k}} \sigma_{i}^{*} \omega$ that represent the singular $k$-chains. Examples are the $k$-submanifolds. The current represents the physical object in $M$ independent of its expression as a combination of simplices.

The physical difference between singular $k$-currents is measured by the flat metric $\left\|\xi_{1}-\xi_{2}\right\|$, $\|\xi\|=\inf \left\{\operatorname{vol}_{k}\left(\xi-\partial \xi^{\prime}\right)+\operatorname{vol}_{k+1}\left(\xi^{\prime}\right): \xi^{\prime} \in \mathcal{D}_{k+1}^{\text {sing }}(M)\right\}$. The space of integral $k$-currents $\mathcal{D}_{k}^{\text {int }}(M)$ is the metric completion: $\mathcal{D}_{k}^{\text {sing }}(M) \subset \mathcal{D}_{k}^{\text {int }}(M) \subset \mathcal{D}_{k}^{\text {distr }}(M)$, a complete metric space and an abelian group. The boundary of an integral current is an integral current.

Recall the $2 \mathrm{~d}$ gaussian model: the free 1 -form $\mathrm{cft}$ in $2 \mathrm{~d} . j(x)$ is a 1-form on a Riemann surface satisfying $d j=0, d(* j)=0$. The integrals of $j$ and $* j$ are 0 -forms $\phi, \phi^{*}$ which take values in dual circles, $d \phi=j, d \phi^{*}=* j, \phi(x) \in \mathbb{R} / 2 \pi R \mathbb{Z}, \phi^{*}(x) \in \mathbb{R} / 2 \pi R^{*} \mathbb{Z}, R R^{*}=1$. They are determined up to $U(1) \times U(1)$ global symmetries $\phi(x) \rightarrow \phi(x)+a, \phi^{*}(x) \rightarrow \phi^{*}(x)+a^{*}$. The vertex operator $V_{p, p^{*}}(x)=e^{i p \phi(x)+i p^{*} \phi^{*}(x)}$ describes a point defect of charges $p, p^{*} \in \frac{1}{R} \mathbb{Z} \times \frac{1}{R^{*}} \mathbb{Z}$. It transforms by $V_{p, p^{*}} \rightarrow V_{p, p^{*}} e^{i p a+i p^{*} a^{*}}$.

Recall the free $n$-form cft in $2 n$ dimensions. $F(x)$ is an $n$-form on the $2 n$-manifold $M$ satisfying $d F=0, d(* F)=0$. The integrals of $F$ and $* F$ are $(n-1)$-forms $A, A^{*}, d A=F, d A^{*}=* F$ which take values in dual circles $\int_{\xi} A \in \mathbb{R} / 2 \pi R \mathbb{Z}, \int_{\xi} A^{*} \in \mathbb{R} / 2 \pi R^{*} \mathbb{Z} \forall \xi \in \mathcal{D}_{n-1}^{\text {sing }}(M), R R^{*}=1 . A, A^{*}$ are determined up to $U(1) \times U(1)$ local gauge symmetries given by $(n-2)$-forms $f, f^{*}, A \rightarrow A+d f$, $A^{*} \rightarrow A^{*}+d f^{*}$. $(n-1)$-dimensional defects are described by fields on $\mathcal{D}_{n-1}^{\text {sing }}(M), V_{p, p^{*}}(\xi)=$ $e^{i p \phi(\xi)+i p^{*} \phi^{*}(\xi)}, \phi(\xi)=\int_{\xi} A, \phi^{*}(\xi)=\int_{\xi} A^{*}, p, p^{*} \in \frac{1}{R} \mathbb{Z} \times \frac{1}{R^{*}} \mathbb{Z}$ transforming by $V_{p, p^{*}}(\xi) \rightarrow$ $V_{p, p^{*}}(\xi) e^{i p a(\partial \xi)+i p^{*} a^{*}(\partial \xi)}, a(\partial \xi)=\int_{\partial \xi} f, a^{*}(\partial \xi)=\int_{\partial \xi} f^{*}$. Fix an (n-2)-boundary $\partial \xi_{0}$ and consider the abelian subgroup $\mathcal{D}_{n-1}^{\text {sing }}(M)_{\mathbb{Z} \partial \xi_{0}}=\left\{\xi: \partial \xi \in \mathbb{Z} \partial \xi_{0}\right\} \subset \mathcal{D}_{n-1}^{\text {sing }}(M)$. On $\mathcal{D}_{n-1}^{\text {sing }}(M)_{\mathbb{Z} \partial \xi_{0}}$ the gauge symmetries act as a global $U(1) \times U(1)$ generated by the numbers $a\left(\partial \xi_{0}\right)$ and $a^{*}\left(\partial \xi_{0}\right)$.

Calculus is needed on $\mathcal{D}_{n-1}^{\text {sing }}(M)_{\mathbb{Z} \partial \xi_{0}}$ to continue the analogy with the $2 \mathrm{~d}$ gaussian model. Go to the metric completion, writing it $Q=\mathcal{D}_{n-1}^{\text {int }}(M)_{\mathbb{Z} \partial \xi_{0}}$. Geometric Measure Theory provides the spaces $\mathcal{D}_{j}^{\text {int }}(Q)$ of integral $j$-currents in any such complete metric space [207]. Define the $j$-forms on $Q$ as the real duals of the currents and the exterior derivative as the dual of the boundary operator, $\Omega_{j}(Q)=\operatorname{Hom}\left(\mathcal{D}_{j}^{\text {int }}(Q), \mathbb{R}\right), d \omega(\eta)=\omega(\partial \eta)$. The infinitesimal $j$-simplices generate $\mathcal{D}_{j}^{\text {int }}(Q)$, so the tangent bundle $T Q$ can be defined as the set of infinitesimal 1-simplices in $Q$. The 1 -forms then become the sections of the dual cotangent bundle $T^{*} Q$. The equivalences of ${ }^{23}$ This section was authored by Daniel Friedan from the New High Energy Theory Center at Rutgers University, Piscataway, New Jersey, USA and the Natural Science Institute at the University of Iceland, Reykjavík, Iceland. 
simplices $\Delta^{j} \times \Delta^{n-1} \cong \Delta^{j+n-1}$ give natural maps $\Pi_{j, n-1}: \mathcal{D}_{j}^{\text {int }}(Q) \rightarrow \mathcal{D}_{j+n-1}^{\text {int }}(M), \partial \Pi_{j, n-1}=$

$\Pi_{j-1, n-1} \partial$. The map $\Pi_{1, n-1}$ identifies each tangent space $T_{\xi} Q$ with a certain subspace $\mathcal{V}_{n} \subset$ $\mathcal{D}_{n}^{\text {distr }}(M)$. That $* \mathcal{V}_{n}=\mathcal{V}_{n}$ is a crucial technical point whose demonstration uses the flat metric completion. Then $*$ acts on each tangent space $T_{\xi} Q$.

The forms $F, * F, A, A^{*}$ pull back to $Q, j=\Pi_{1, n-1}^{*} F, * j=\Pi_{1, n-1}^{*}(* F), \phi=\Pi_{0, n-1}^{*} A, \phi^{*}=$ $\Pi_{0, n-1}^{*} A^{*}$, with $d \phi=j, d \phi^{*}=* j$. So there is the classical $2 \mathrm{~d}$ gaussian model on each of the spaces $Q$, except that $*^{2}=1$ for $n$ even, while $*^{2}=-1$ in $2 \mathrm{~d}$. Define $J=\epsilon_{n} *, \epsilon_{n}^{2}=(-1)^{n-1}$, so $J^{2}=-1$. $J$ is imaginary when $n$ is even, so the currents have to be complexified in order that $J$ act on the tangent spaces $T_{\xi} Q, Q=\mathcal{D}_{n-1}^{\text {int }}(M)_{\mathbb{Z} \partial \xi_{0}} \oplus i \partial \mathcal{D}_{n}^{\text {int }}(M)$. Now, for all $n$, on each of the spaces $Q$ there is a $2 d$ gaussian model written in terms of the chiral fields $d \phi_{ \pm}=j_{ \pm}, j_{ \pm}=\frac{1}{2}\left(1 \pm i^{-1} J\right) j$.

Quantization of a free field theory is expressed by the Schwinger-Dyson equation on the 2-point functions. In the $2 \mathrm{~d}$ gaussian model, the chiral fields are (anti-)holomorphic. The S-D equation on $\left\langle\bar{\phi}_{ \pm} j_{ \pm}\right\rangle$is the Cauchy-Riemann equation $\frac{\partial}{\partial \bar{z}} \frac{1}{z-z^{\prime}}=\pi \delta^{2}\left(z-z^{\prime}\right)$ which is the foundation for complex analysis on Riemann surfaces. The $2 \mathrm{~d}$ gaussian model would have led to complex analysis on Riemann surfaces had that not existed already. For the free $n$-form in $2 n$ dimensions, the S-D equation has an expression containing no explicit mention of $n,\left\langle\int_{\bar{\xi}_{1}} \bar{A}_{\alpha} \int_{\xi_{2}} d F_{\beta}\right\rangle=2 \pi i c_{\alpha \beta} I_{M}\left\langle\bar{\xi}_{1}, \xi_{2}\right\rangle, c_{\alpha \beta}=-c_{\beta \alpha}, c_{+-}=1$. The lhs is the 2pt function $\left\langle\bar{A}_{ \pm}(x) d F_{ \pm}\left(x^{\prime}\right)\right\rangle$ smeared against the $(n-1)$-current $\bar{\xi}_{1}$ and the $(n+1)$-current $\xi_{2}$. The rhs is a slight modification of the intersection number, which is nonzero only if $k_{1}+$ $k_{2}=2 n, I_{M}\left(\xi_{1}, \xi_{2}\right)=\int_{M} \frac{1}{k_{1} ! k_{2} !} \xi_{1}^{\mu_{1} \cdots}(x) \xi_{2}^{\nu_{1} \cdots \mu_{k_{1}}}(x) \epsilon_{\mu_{1} \cdots \mu_{k_{1}} \nu_{1} \cdots \nu_{k_{2}}}(x) d^{2 n} x$. The modification $I_{M}\left\langle\bar{\xi}_{1}, \xi_{2}\right\rangle=\epsilon_{n, k_{2}-n} I_{M}\left(\bar{\xi}_{1}, \xi_{2}\right), \epsilon_{n, m}=(-1)^{n m+m(m+1) / 2} \epsilon_{n}^{-1}$ is such that $I_{M}\left\langle\bar{\xi}_{1}, \xi_{2}\right\rangle$ has properties independent of $n: I_{M}\left\langle\bar{\xi}_{1}, \xi_{2}\right\rangle$ is skew-hermitian; $I_{M}\left\langle\bar{\partial}_{1}, \xi_{2}\right\rangle=-I_{M}\left\langle\bar{\xi}_{1}, \partial \xi_{2}\right\rangle$; $I_{M}\left\langle\bar{\xi}_{1}, J \xi_{2}\right\rangle$ on $n$-currents is hermitian and positive definite.

Pulled back to $Q$, the S-D equation of the free $n$-form cft is $\left\langle\int_{\bar{\eta}_{1}} \bar{\phi}_{\alpha} \int_{\eta_{2}} d j_{\beta}\right\rangle=2 \pi i c_{\alpha \beta} I_{Q}\left\langle\bar{\eta}_{1}, \eta_{2}\right\rangle$. The rhs is $I_{M}\left\langle\bar{\xi}_{1}, \xi_{2}\right\rangle$ pulled back to a skew-hermitian form on currents in $Q, I_{Q}\left\langle\bar{\eta}_{1}, \eta_{2}\right\rangle=$ $I_{M}\left\langle\Pi_{j_{1}, n-1} \bar{\eta}_{1}, \Pi_{j_{2}, n-1} \eta_{2}\right\rangle$ which is nonzero only if $\left(j_{1}+n-1\right)+\left(j_{2}+n-1\right)=2 n$, which is $j_{1}+j_{2}=2$, just like the intersection number of currents in a 2-manifold. The S-D equation on $Q$ is formally analogous to the S-D equation of the $2 \mathrm{~d}$ gaussian model on a Riemann surface, which is the Cauchy-Riemann equation.

The free $n$-form cft on $M$ has now become the $2 \mathrm{~d}$ gaussian model on each of the metric spaces $Q$. Moreover, each $Q$ has the structure needed to write the Cauchy-Riemann equation. This is taken to be the defining structure of a quasi Riemann surface. The $Q=\mathcal{D}_{\mathbb{Z} \partial \xi_{0}}^{\text {int }}$ are the quasi Riemann surfaces. They are the fibers of a bundle of quasi Riemann surfaces $\mathcal{Q}(M) \rightarrow \mathcal{B}(M)$, $\mathcal{B}(M)=\left\{\right.$ maximal infinite cyclic subgroups $\left.\mathbb{Z} \partial \xi_{0} \subset \partial \mathcal{D}_{n-1}^{\text {int }}(M)\right\}$. On each fiber $Q$ there is a $2 \mathrm{~d}$ gaussian model with its global $U(1) \times U(1)$ symmetry group, collectively comprising a local gauge symmetry over $\mathcal{B}(M)$.

All of the constructions of $2 \mathrm{~d} \mathrm{cft}$ are based on the Cauchy-Riemann equation and on the $2 \mathrm{~d}$ gaussian model. So there is the prospect of carrying out those constructions on each of the fibers $Q$ to obtain, for every $2 \mathrm{~d} \mathrm{cft}$, a new cft of defects in $M$. The $2 \mathrm{~d} \mathrm{ctt}$ on each fiber $Q$ will be ambiguous up to its global $2 \mathrm{~d}$ symmetry group. The collection of global symmetry groups on the fibers will forms a local gauge symmetry group over $\mathcal{B}(M)$.

There are many basic problems to be worked on: opportunities to leverage $2 \mathrm{~d}$ qft to develop a new technology of $\mathrm{qft}$ in $2 n$ dimensions. Some are the following.

Complex analysis on quasi Riemann surfaces needs to be developed in analogy with ordinary Riemann surfaces.

Conjecturally, every quasi Riemann surface $Q$ is isomorphic to $\mathcal{D}_{0}^{\text {int }}(\Sigma)$ for $\Sigma$ the 2 d conformal space with the same Jacobian as $Q$, the Jacobian being the complex torus made from the homology in the middle dimension.

The conjectured isomorphism would allow constructing a $2 \mathrm{~d}$ cft on each $Q$ by lifting an ordinary $2 \mathrm{~d}$ cft from $\Sigma$ to $\mathcal{D}_{0}^{\text {int }}(\Sigma)$, which is a purely $2 \mathrm{~d}$ problem, then to each $Q=\mathcal{D}^{\text {int }}(M)_{\mathbb{Z} \partial \xi_{0}}$ via one of the conjectured isomorphisms. As an example of the first step, the $2 \mathrm{~d}$ gaussian model

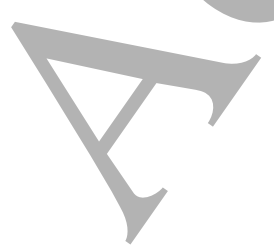


is to be lifted by extending the renormalization of the vertex operators $V_{p, \bar{p}}(\xi)$ from singular 0 -currents $\xi$ to integral 0 -currents.

If the conjecture is true, there will be some universal objects to study. The automorphism group $\operatorname{Aut}(Q)$ will encode information about all the conformal groups of the conformal manifolds $M$ and about the global symmetry groups of all $2 \mathrm{~d}$ cfts. There will be a universal homogeneous bundle of quasi Riemann surfaces with structure group $\operatorname{Aut}(Q)$ in which all the bundles $\mathcal{Q}(M) \rightarrow$ $\mathcal{B}(M)$ are embedded.

There should be a large collection of structure preserving maps from the complex disk $\mathbb{D}_{1}$ into $Q$. Meromorphic functions on $Q$ will pull back to ordinary meromorphic functions on $\mathbb{D}_{1}$. The local structure of a cft on $Q$ will be expressed as a collection of ordinary $2 \mathrm{~d} \mathrm{ctts}$ on each of these local quasi holomorphic curves, each with its radial quantization, pair of Virasoro algebras, and operator product expansion. Explicit constructions of local quasi holomorphic curves are needed, say for $M=S^{2 n}$.

The local gauge symmetry in the bundle of quasi Riemann surfaces needs a space-time interpretation. What, for example, is the space-time interpretation of the local $S U(2) \times S U(2)$ symmetry over $\mathcal{B}(M)$ corresponding to the global $S U(2) \times S U(2)$ at the self-dual point $R=1$ of the $2 \mathrm{~d}$ gaussian model?

It should be possible to imitate on the quasi Riemann surfaces the usual constructions of $2 \mathrm{~d}$ cft such as orbifolding and perturbation theory.

Tiny defects look like points in $M$, so fields $\Phi(\xi)$ when restricted to the small $\xi$ in $Q$ will give ordinary local quantum fields on $M$. Will these form new local qfts in $2 n$-dimensions?

Acknowledgements. All of the authors would like to acknowledge the Royal Society for providing the opportunity to participate in the workshop. AB would like to thank Fernando Alday for collaboration on [130] and related topics. PED would like to thank Vincent Caudrellier, Ed Corrigan, Giuseppe Mussardo, Tomasz Romanczukiewicz and Yasha Shnir for helpful discussions as well as Matt Buican and Andy O'Bannon for the invitation to speak at such an enjoyable workshop. JE would like to thank Andy O'Bannon, Carlos Hoyos, Mario Flory, Max Newrzella, Jackson Wu, and Ioannis Papadimitriou for fruitful collaboration and the participants of the Workshop for stimulating discussions. CK would like to hank the organizers of the workshop "Boundary and Defect Conformal Field Theory: Open Problems and Applications" for the invitation to speak there and for creating an inspiring atmosphere and thank all of her collaborators for illuminating discussions. SR would like to acknowledge discussions with Gil Young Cho, Andreas Ludwig, Ken Shiozaki, Bo Han, Apoorv Tiwari, Chang-Tse Hsieh, Xueda Wen, Shunji Matsuura, Shoucheng Zhang, Masamichi Miyaji, Tadashi Takayanagi, and Takahiro Morimoto.

\section{References}

1. Affleck I. 1995. Conformal field theory approach to the Kondo effect. Acta Phys. Polon. B 26, 1869 (1995) [cond-mat/9512099].

2. Recknagel A., and Schomerus V. "Boundary Conformal Field Theory and the Worldsheet Approach to D-Branes," doi:10.1017/CBO9780511806476

3. Herzog C.P., Huang K-W. 2017. Boundary Conformal Field Theory and a Boundary Central Charge. JHEP 1710189.

4. Herzog C.P., Huang K-W., Jensen K. 2017 Displacement Operators and Constraints on Boundary Central Charges, e-Print: arXiv:1709.07431 [hep-th].

5. Cardy J. 2017. Bulk Renormalization Group Flows and Boundary States in Conformal Field Theories. SciPost Phys. 3, 011.

6. Cardy J and Lewellen D. 1991. Bulk and Boundary Operators in Conformal Field Theory. Phys. Lett. B 259, 274.

7. Konechny A. 2017. RG boundaries and interfaces in Ising field theory. J. Phys. A 50, 145403.

8. Yurov V.P and Zamolodchikov Al.B. 1990. Truncated Conformal Space Approach to Scaling Lee-Yang Model. Int. J. Mod. Phys. A 5, 3221.

9. Hogervorst M, Rychkov S, van Rees BC. 2015. A Cheap Alternative to the Lattice? Phys. Rev. D 91, 025005 .

10. Konechny A. 2017. RG boundaries and interfaces in Ising field theory. J. Phys. A50 14. 
11. Brunner I. and Roggenkamp D. 2008. Defects and bulk perturbations of boundary LandauGinzburg orbifolds. JHEP 080400.

12. Fonseca P. and Zamolodchikov A. 2003 Ising field theory in a magnetic field: Analytic properties of the free energy. J. Stat. Phys. 1103.

13. Quella T., Runkel I. and Watts G. 2007. Reflection and Transmission for Conformal Defects. JHEP 0704095.

14. Klebanov I.R., Witten E. 1999. AdS / CFT correspondence and symmetry breaking. Nucl. Phys. B 556:89.

15. Melby-Thompson C.M., Schmidt-Colinet C. 2017. Double trace interfaces. JHEP 2017110

16. Bak D, Gutperle M, Hirano S. 2003. A dilatonic deformation of AdS(5) and its field theory dual. JHEP 0305:072.

17. McAvity D.M., Osborn H. 1995. Conformal field theories near a boundary in general dimensions. Nucl. Phys. B 455:522.

18. Aharony O., DeWolfe O., Freedman D.Z., Karch A. 2003. Defect conformal field theory and locally localized gravity. JHEP 0307:030.

19. Diaz D.E., Dorn H. 2007. Partition functions and double-trace deformations in AdS/CFT. JHEP 0705:046.

20. Gaberdiel M.R., Gopakumar R. 2013. Minimal Model Holography. J. Phys. A46:214002.

21. Gaiotto D. 2012. Domain walls for two-dimensional renormalization group flows. JHEP 12:103.

22. Sneddon I.N. 1966. Mixed Boundary Value Problems in Potential Theory. Amsterdam: NorthHolland Publishing Company.

23. Cardy J.. 2004. Boundary Conformal Field Theory. e-Print: arXiv: hep-th/0411189.

24. Cho G.Y., Ludwig A.W.W. and Ryu S.. 2017. Universal entanglement spectra of gapped onedimensional field theories. Phys. Rev. B95 11.

25. Cho, G.Y., Shiozaki K., Ryu S., and Ludwig A.W.W.. 2017. Relationship between symmetry protected topological phases and boundary conformal field theories via the entanglement spectrum. J. Phys. A Math. Gen. 50.

26. Han B., Tiwari A., Hsieh C-T. and Ryu S.. 2017. Boundary conformal field theory and symmetry-protected topological phases in $2+1$ dimensions. Phys. Rev. B96 12.

27. Fliss J.R., Wen X., Parrikar O., Hsieh C.-T., Han B., Hughes TL. and Leigh RG. 2017. Interface contributions to topological entanglement in abelian Chern-Simons theory. JHEP 9056.

28. Wong G.. 2017. A note on entanglement edge modes in Chern Simons theory. e-Print: arXiv: 1706.04666.

29. Wen X., Matsuura S. and Ryu S.. 2016. Edge theory approach to topological entanglement entropy, mutual information, and entanglement negativity in Chern-Simons theories. Phys. Rev. B93 24.

30. Qi, X-L., Katsura H. and Ludwig A.W.W.. 2012. General Relationship between the Entanglement Spectrum and the Edge State Spectrum of Topological Quantum States. Phys. Rev. Lett. 10819.

31. Chen X., Gu Z-C., Liu Z-X., and Wen X.-G... 2013. Symmetry protected topological orders and the group cohomology of their symmetry group. Phys. Rev. B87 15.

32. Ryu S. and Zhang S-C.. 2012. Interacting topological phases and modular invariance. Phys. Rev. B85 24.

33. Konechny A.. 2017. RG boundaries and interfaces in Ising field theory. J. Phys. A Math.Gen. 50 14.

34. Miyaji M,, Ryu S., Takayanagi T. and Wen X.. 2015. Boundary states as holographic duals of trivial spacetimes. JHEP 5152.

35. Cho G.Y., Hsieh C-T., Morrimoto T. and Ryu, S. 2015. Topological phases protected by reflection symmetry and cross-cap states. Phys. Rev.. B91 19.

36. Kane C.L. and Fisher M.P.A. 1992. Transmission through barriers and resonant tunneling in an interacting one-dimensional electron gas. Phys. Rev. B 46, 23.

37. Giamarchi T. 2003 Quantum Physics in One Dimension. Clarendon Press.

38. Furusaki A. and Nagaosa N. 1993. Resonant tunneling in a Luttinger liquid. Phys. Rev. B 47, 7.

39. Komnik A. and Gogolin A.O. 2003. Resonant Tunneling between Luttinger Liquids: A Solvable Case. Phys. Rev. Lett. 90, 24.

40. Furusaki A. and Matveev K.A. 2002. Occupation of a Resonant Level Coupled to a Chiral

Luttinger Liquid. Phys. Rev. Lett. 88, 22. 
41. Furusaki A. 1998. Resonant tunneling through a quantum dot weakly coupled to quantum wires or quantum Hall edge states. Phys. Rev. B 57, 12.

42. Goldstein M.,Weiss Y. and Berkovits R. 2009. Interacting resonant level coupled to a Luttinger liquid: Universality of thermodynamic properties. EPL $\mathbf{8 6}$.

43. Goldstein M. and Berkovits R. 2010. Duality between Different Geometries of a Resonant Level in a Luttinger Liquid. Phys. Rev. Lett. 104, 10.

44. Goldstein M. and Berkovits R. 2010. Capacitance of a resonant level coupled to Luttinger liquids. Phys. Rev. B 82, 16.

45. Kakashvili P. and Johannesson H. 2003. Measuring Luttinger Liquid Correlations from Charge Fluctuations in a Nanoscale Structure. Phys. Rev. Lett 91, 18.

46. Wächter P., Menden V. and Schönhammer K. 2007. Charging of a quantum dot coupled to Luttinger-liquid leads. Phys. Rev. B 76, 12.

47. Lerner I.V., Yudson V.I. and Yurkevich I.V. 2008. Quantum Wire Hybridized With a SingleLevel Impurity. Phys. Rev. Lett. 100, 25.

48. Haldane F.H.M. 1981. Effective harmonic-fluid approach to low-energy properties of onedimensional quantum fluids. Phys. Rev. Lett 47, 25.

49. Bockrath M. ,Cobden, DH., Lu J., Rinzler AG., Smalley RE., Balents L., and McEuen PL. Luttinger-liquid behaviour in carbon nanotubes. Nature 397.

50. Yao Z., Postma H.W.C., Balents L., and Dekker C. 1999. Carbon nanotube intramolecular junctions. Nature 402.

51. Chang A.M. 2003. Chiral Luttinger liquids at the fractional quantum Hall edge. Rev. Mod. Phys. 75, 4 .

52. Milliken F.P., Umbach C.P. and Webb R.A. 1996. Indications of a Luttinger liquid in the fractional quantum Hall regimet. Solid State Comm. 97.

53. Maasilta I.J., and Goldman V.J. 1997. Line shape of resonant tunneling between fractional quantum Hall edges. Phys Rev. B 557.

54. Citro R., Orignac E., De Palo S. and Chiofalo M.L. 2007. Evidence of Luttinger-liquid behavior in one-dimensional dipolar quantum gases. Phys. Rev. A 755.

55. Recati A., Fedichev P.O., Zwerger W., von Delft J. and Zoller P. 2005. Atomic Quantum Dots Coupled to a Reservoir of a Superfluid Bose-Einstein Condensate. Phys. Rev. Lett. 944.

56. Jiang L., Baksmaty L.O., Hu H., Chen Y. and Pu H. 2011. Single impurity in ultracold Fermi superfluids. Phys. Rev. A 85, 6.

57. Bloch I., Dalibard J. and Zwerger W. 2009. Many-body physics with ultracold gases. Mod. Rev. Phys 803.

58. Del Maestro A., Boninsegni M., and Affleck I. 2011. ${ }^{4}$ He. Phys. Rev. Lett 10610.

59. Duc P.F., Savard M., Petrescu M., Rosenow B., Del Maestro A. and Gervais G. 2015. Critical flow and dissipation in a quasi-one-dimensional superfluid. Sci. Adv. 1.

60. Safi I. and Saleur H. 204.One-Channel Conductor in an Ohmic Environment: Mapping to a Tomonaga-Luttinger Liquid and Full Counting Statistics. Phys. Rev. Lett. 59312.

61. Le Hur K. and Li M-R. 2005. Unification of electromagnetic noise and Luttinger liquid via a quantum dot. Phys Rev. B 727.

62. Mebrahtu H.T., Brozenets I.V., Liu D.E., Zheng H., Bomze Y.V., Smirnov A.I., Baranger H.U. and Finkelstein G. 2012 Quantum phase transition in a resonant level coupled to interacting leads. Nature 4887409.

63. Mebrahtu H.T., Borzenets I.V., Zheng H., Bomze Y.V., Smirnov A.I., Florens S., Baranger H.U. and Finkelstein G. 2013. Observation of Majorana quantum critical behaviour in a resonant level coupled to a dissipative environment. Nature Physics 9.

64. Andrei N. 1992. Integrable Models in Condensed Matter Physics. World Scientific.

65. Tsvelick A.M. and Wiegmann PB. 1983. Exact results in the theory of magnetic alloys. Advances in Phys. 32.

66. Rylands C. and Andrei N. 2016. Quantum impurity in a Luttinger liquid: Exact solution of the Kane-Fisher model. Phys. Rev. B 9411.

67. Zamolodchikov AB. 1991. TBA equations for integrable perturbed $\mathrm{SU}(2)_{k} \times \mathrm{SU}(2) 1 \mathrm{SU}(2)_{k+1}$ coset models. Nucl. Phys. B 3661.

68. Fendley P. and Saleur H. and Warner NP. 1994. Exact solution of a massless scalar field with a relevant boundary interaction. Nucl. Phys. B 430.

69. Rylands C. and Andrei N. 2017. Quantum Dot in Interacting Environments. arXiv: 1708.07212

[cond-mat.str-el]. 
70. Ghoshal S., and Zamolodchikov A. 1994. Boundary S-matrix and boundary state in twodimensional integrable quantum field theory. Int. J. Mod. Phys. A 9 3841-3886.

71. MacIntyre A. 1995. Integrable boundary conditions for classical sine-Gordon theory. J. Phys. A 28 1089-1100.

72. Arthur R., Dorey P., and Parini R. 2016. Breaking integrability at the boundary: the sineGordon model with Robin boundary conditions. J. Phys. A 49 165205. [arXiv:1509.08448 [hep-th]].

73. See the solitonscattering GitHub repository at https://github.com/rparini/solitonscattering

74. Campbell D.K., Schonfeld J.F., and Wingate C.A. 1983. Resonance structure in kink-antikink interactions in $\phi^{4}$ theory. Physica D 91.

75. Anninos P., Oliveira S., and Matzner R.A. 1991. Fractal structure in the scalar $\lambda\left(\phi^{2}-1\right)^{2}$ theory. Phys. Rev. D 441147.

76. Goodman R., and Haberman R. 2007. Chaotic scattering and the $n$-bounce resonance in solitary wave interactions. Phys. Rev. Lett. 98104103.

77. Dorey P., Mersh K., Romanczukiewicz T., and Shnir Y. 2011. Kink-antikink collisions in the $\phi^{6}$ model. Phys. Rev. Lett. 107091602.

78. Dorey P., Halavanau A., Mercer J., Romanczukiewicz T., and Shnir Y. 2015. Boundary scattering in the $\phi^{4}$ model. JHEP 1705107.

79. Pelloni B. 2015. Advances in the study of boundary value problems for nonlinear integrable PDEs. Nonlinearity 282.

80. Delfino G., Mussardo G., and Simonetti P. 1996. Nonintegrable quantum field theories as perturbations of certain integrable models. Nucl. Phys. B 473469.

81. Petkova V.B. and Zuber J-B. 2001. Generalized twisted partition functions. Phys. Lett. B504.

82. Gang D. and Yamaguchi S. 2008. Superconformal defects in the tricritical Ising model. JHEP 2008076.

83. Makabe I. and Watts G.M.T. 2017. Defects in the Tri-critical Ising model. JHEP 2008 076, ePrint: arXiv:1703.09148.

84. Novak S. and Runkel I. 2015.Spin from defects in two-dimensional quantum field theory. ePrint: arXiv: 1506.07547.

85. Komargodski S., Seiberg N. 2010. Comments on Supercurrent Multiplets, Supersymmetric Field Theories and Supergravity. JHEP 1007017.

86. Drukker N., Martelli D., and Shamir I. 2017. The energy-momentum multiplet of supersymmetric defect field theories. JHEP 1708010.

87. Karch A. and Randall L. 2001. Locally localized gravity.JHEP 0105008.

88. DeWolfe O., Freedman D.Z. and Ooguri H. 2002. Holography and defect conformal field theories. Phys. Rev. D66

89. Erdmenger J., Guralnik Z. and Kirsch I. 2002. Four-dimensional superconformal theories with interacting boundaries or defects. Phys. Rev. D66

90. Constable N.R., Myers R.C. and Tafjord O. 2000. The Noncommutative bion core. Phys. Rev. D61.

91. de Leeuw M., Kristjansen C. and Zarembo K. 2015. One-point Functions in Defect CFT and Integrability, JHEP 1508098.

92. Buhl-Mortensen I., de Leeuw M., Kristjansen C. and Zarembo K. 2016. One-point Functions in AdS/dCFT from Matrix Product States. JHEP 1602052.

93. Brockmann M. 2014. Overlaps of $q$-raised Néel states with XXZ Bethe states and their relation to the Lieb-Liniger Bose gas. J. Stat. Mech: Theor. and Exp. 5.

94. de Leeuw M., Kristjansen C. and Mori S. 2016. AdS/dCFT one-point functions of the SU(3) sector. Phys. Lett. B763 197.

95. De Leeuw M., Kristjansen C. and Linardopoulos G. 2018. Scalar one-point functions and matrix product states of AdS/dCFT. Phys. Lett. B 781238.

96. Buhl-Mortensen I., de Leeuw M., Ipsen A.C., Kristjansen C., and Wilhelm M. 2017. Asymptotic One-Point Functions in Gauge-String Duality with Defects. Phys. Rev. Lett. 119$) 26$.

97. Piroli L.,Pozsgay B. and Vernier E. 2017. What is an integrable quench? Nucl. Phys B925 362.

98. Nagasaki K., Tanida H. and Yamaguchi S. 2012. Holographic Interface-Particle Potential. JHEP 1201139.

99. Nagasaki K. and Yamaguchi S. 2012. Expectation values of chiral primary operators in holographic interface CFT. Phys. Rev. D86.

100. Buhl-Mortensen I., de Leeuw M., Ipsen AC., Kristjansen C. and Wilhelm M. 2016. One-loop one-point functions in gauge-gravity dualities with defects. Phys. Rev. Lett 11723. 
101. Buhl-Mortensen I., de Leeuw M., Ipsen AC., Kristjansen C. and Wilhelm M. 2017. A Quantum Check of AdS/dCFT. JHEP 1701098.

102. Liendo P. and Meneghelli C. 2017. Bootstrap equations for $\mathcal{N}=4$ SYM with defects. JHEP 1701122

103. de Leeuw M., Ipsen AC., Kristjansen C. ,Vardinghus KE. and Wilhelm M. 2017. Two-point functions in AdS/dCFT and the boundary conformal bootstrap equations. JHEP 1708020.

104. de Leeuw M., Kristjansen C. and Linardopoulos G. 2017. One-point functions of nonprotected operators in the SO(5) symmetric D3/D7 dCFT. J. Phys A50 25.

105. Kristjansen C., Semenoff GW. and Young D. 2013. Chiral primary one-point functions in the D3-D7 defect conformal field theory. JHEP 1301117.

106. Kondo J. 1964. Resistance Minimum in Dilute Magnetic Alloys. Progress of Theoretical Physics 321.

107. Erdmenger J., Hoyos C., O’Bannon A., and Wu J. 2013. A Holographic Model of the Kondo Effect. JHEP 12086.

108. Hartnoll S. A., Herzog C. P., and Horowitz G. T. 2008. Building a Holographic Superconductor. Phys. Rev. Lett. 101031601.

109. Read N., and Newns D. M. 1983. On the solution of the Coqblin-Schrieffer Hamiltonian by the large-N expansion technique. J. Phys. C 1617.

110. Coleman P., and Andrei N. 1986. Diagonalisation of the generalised Anderson model. J. Phys. C 1917.

111. Ryu S., and Takayanagi T. 2006. Holographic derivation of entanglement entropy from AdS/CFT. Phys. Rev. Lett. 96181602.

112. Calabrese P., and Cardy J. L.. 2004. Entanglement entropy and quantum field theory. J. Stat. Mech. 0406 P06002.

113. Erdmenger J., Flory M., and Newrzella M.-N. 2015. Bending branes for DCFT in two dimensions. JHEP 01058.

114. Erdmenger J., Flory M., Hoyos C., Newrzella M.-N., and Wu J. M. S. 2016. Entanglement Entropy in a Holographic Kondo Model. Fortsch. Phys. 64 109-130.

115. Israel W. 1966. Singular hypersurfaces and thin shells in general relativity. Nuovo Cim. B44 1.

116. Sørensen E.S., Chang M.-S., Laflorencie N., and Affleck I. 2007. Quantum impurity entanglement. J. Stat. Mech 06 P08003.

117. Erdmenger J. 2017. Introduction to Gauge/Gravity Duality (TASI Lectures 2017). PoS TASI2017 001.

118. Erdmenger J., Flory M., Newrzella M.-N., Strydom M., and Wu J. M. S. 2017. Quantum Quenches in a Holographic Kondo Model. JHEP. 04045.

119. Erdmenger J., Hoyos C., O'Bannon A., Papadimitriou I., Probst J., and Wu J. M. S. 2017. Holographic Kondo and Fano Resonances. Phys. Rev. D96 2.

120. Erdmenger J., Hoyos C., O’Bannon A., Papadimitriou I., Probst J., and Wu J. M. S. 2017. Two-point Functions in a Holographic Kondo Model. JHEP 03039.

121. Maldacena J.M. 1999. The Large N limit of superconformal field theories and supergravity. Int. J. Theor. Phys. 38.

122. Gubser SS., Klebanov IR. and Polyakov AI. 1998. Gauge theory correlators from noncritical string theory. Phys. Lett. B429.

123. Witten E. 1998. Anti-de Sitter space and holography. Adv. Theor. Math. Phys. 2.

124. Freedman D.Z., Mathur S.D., Matusis A., and Rastelli L. 1999. Correlation functions in the CFT(d) / AdS (d+1) correspondence. Nucl. Phys. B546.

125. D'Hoker E., Freedman D.Z. and Skiba W. 1999. Field theory tests for correlators in the AdS / CFT correspondence. Phys. Rev. D59.

126. Rastelli L and Zhou X. 2017. Mellin amplitudes for $A d S_{5} \times S^{5}$. Phys. Rev. Lett 118, 9 .

127. Rastelli L. and Zhou X. 2017. How to Succeed at Holographic Correlators Without Really Trying.

128. Dolan FA., Nirschl M. and Osborn, H. 2006. Conjectures for large N superconformal N=4 chiral primary four point functions. Nucl. Phys. B749.

129. Uruchurtu LI. 2011. Next-next-to-extremal Four Point Functions of N=4 1/2 BPS Operators in the AdS/CFT Correspondence. JHEP 08133.

130. Alday LF and Bissi A. 2017. Loop Corrections to Supergravity on $A d S_{5} \times S^{5}$. Phys. Rev. Lett 11917.

131. Komargodski Z. and Zhivoedov A. 2013. Convexity and Liberation at Large Spin. JHEP 11 140.

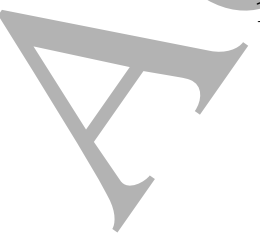


132. Fitzpatrick A.L., Kaplan J., Poland D. and Simmons-Duffin D. 2012. The Analytic Bootstrap and AdS Superhorizon Locality. JHEP 12004.

133. Alday L.F., Bissi A. and Lukowski T. 2015. Lessons from crossing symmetry at large N. JHEP 06074.

134. Alday L.F. and Zhivoedov A. 2017. An Algebraic Approach to the Analytic Bootstrap. JHEP 04157.

135. Alday L.F. 2017. Large Spin Perturbation Theory for Conformal Field Theories. Phys. Rev. Lett 11911.

136. Caron-Huot S. 2017. Analyticity in Spin in Conformal Theories. JHEP 09078.

137. Alday L.F., Bissi A. and Lukowski T. 2015. Large spin systematics in CFT. JHEP 11101.

138. Beem C., Rastelli L. and van Rees BC. 2013. The $\mathcal{N}=4$ Superconformal Bootstrap. Phys. Rev. Lett 111.

139. Beem C., Rastelli L. and van Rees BC. 2017. More $\mathcal{N}=4$ superconformal bootstrap. Phys. Rev. D96 4.

140. Arutyunov G., Frolov S. and Petkou A.C. 1998. Operator product expansion of the lowest weight CPOs in $\mathcal{N}=4 \mathrm{SYM}_{4}$ at strong coupling. Nucl. Phys. B586.

141. D'Hoker E., Mathur S.D., Matusis A. and Rastelli L. 2000. The Operator product expansion of N=4 SYM and the 4 point functions of supergravity. Nucl. Phys. B549.

142. Aharony O., Alday LF, Bissi A. and Perlmutter E. 2017. Loops in AdS from Conformal Field Theory. JHEP 07036.

143. Metsaev R.R. and Tseytlin A.A. 1988. ON LOOP CORRECTIONS TO STRING THEORY EFFECTIVE ACTIONS. Nucl. Phys. B298.

144. Alday L.F. and Caron-Huot S. 2017. Gravitational S-matrix from CFT dispersion relations.

145. Aprile F., Drummond J.M., Heslop P. and Paul H. 2017. Unmixing Supergravity.

146. Aprile F., Drummond J.M., Heslop P. and Paul H. 2017. Loop corrections for Kaluza-Klein AdS amplitudes.

147. Aprile F., Drummond J.M., Heslop P. and Paul H. 2017. Quantum Gravity from Conformal Field Theory.

148. Goçalves V. Four point function of $\mathcal{N}=4$ stress-tensor multiplet at strong coupling. JHEP 04 150.

149. Alday L.F., Bissi A. and Perlmutter E. 2017. Holographic Reconstruction of AdS Exchanges from Crossing Symmetry. JHEP 08147

150. Yuan E. Y. 2017 Loops in the Bulk.

151. Bargheer T., Caetano J, Fleury T., Komatsu S. and Vieira P. 2017. Handling Handles I: Nonplanar Integrability.

152. Dowker J.S., Schofield J.P. 1990. Conformal Transformations and the Effective Action in the Presence of Boundaries. J. Math. Phys. 31808.

153. Herzog C.P., Huang K-W., Jensen K. 2016. Universal Entanglement and Boundary Geometry in Conformal Field Theory. JHEP 1601162.

154. Fursaev D.V. 2015. Conformal Anomalies of CFTs with Boundaries. JHEP 1512112.

155. Tseytlin A.A., Beccaria M. 2017. $C_{T}$ for conformal higher spin fields from partition function on conically deformed sphere. JHEP 1709123.

156. Crardy J. 1988. Is There a c Theorem in Four-Dimensions? Phys. Lett. B215 749.

157. Komargodski Z., Schwimmer A. 2011. On Renormalization Group Flows in Four Dimensions. JHEP 1112 099.

158. Solodukhin S.N. 2016. Boundary terms of conformal anomaly. Phys. Lett. B752 131.

159. Osborn H., Petkou A.C. 1994. Implications of conformal invariance in field theories for general dimensions. Annals Phys. 231, 311-362.

160. Deutsch D., Candelas P. 1979. Boundary Effects in Quantum Field Theory. Phys. Rev. D20, 3063.

161. Rong-Xin M., Chong-Sun C. 2017 Universality for Shape Dependence of Casimir Effects from Weyl Anomaly. e-Print: arXiv:1706.09652 [hep-th].

162. Fursaev D.V. 2006. Entanglement entropy in critical phenomena and analogue models of quantum gravity. Phys. Rev. D73 124025.

163. Fursaev D.V. 2013. Quantum Entanglement on Boundaries. JHEP 1307119.

164. Fursaev D.V., Patrushev A., Solodukhin S.N. 2013. Distributional Geometry of Squashed Cones. Phys. Rev. D88 no.4, 044054.

165. Amin F.A., Berthiere C., Fursaev D.V., Solodukhin S.N. 2017. Holographic calculation of entanglement entropy in the presence of boundaries. Phys. Rev. D95 no.10, 106013. 
166. Takayanagi T. 2011.Holographic Dual of BCFT. Phys. Rev. Lett 107101602.

167. Rong-Xin M., Chong-Sun C., Wu-Zhong G. 2017 New proposal for a holographic boundary conformal field theory. Phys. Rev. D96 4.

168. Astaneh F.A., Solodukhin SN. 2017. Holographic calculation of boundary terms in conformal anomaly. Rev. Lett B769 25.

169. Ryu S., Takayanagi T. 2006. Holographic derivation of entanglement entropy from AdS/CFT. Phys. Rev. Lett 96.

170. Schwimmer A., Theisen S. 2008. Entanglement Entropy, Trace Anomalies and Holography Nucl. Phys. B801 1.

171. Runkel I., Fjelstad J., Fuchs J. and Schweigert C. 2007. Topological and conformal field theory as Frobenius algebras. Contemp. Math. 431225.

172. Polyakov A.M. 1974. Nonhamiltonian approach to conformal quantum field theory. Zh. Eksp. Teor. Fiz. 6623.

173. Mack G.1973. Conformal Invariant Quantum Field Theory. J. Phys. Colloq. 34 C1, 99.

174. Ferrara S., Grillo A.F., Parisi G. and Gatto R. 1972. Covariant expansion of the conformal four-point function. Nucl. Phys. B49 77.

175. Dolan F.A. and Osborn H. 2001. Conformal four point functions and the operator product expansion. Nucl. Phys. B599 459.

176. Dolan F.A. and Osborn H. 2004. Conformal partial waves and the operator product expansion. Nucl. Phys. B678 491.

177. Dolan F.A. and Osborn H. 2011. Conformal Partial Waves: Further Mathematical Results. e-Print: arXiv:1108.6194.

178. Billo M., Goncalves V., Lauria E. and Meineri M. 2016. Defects in conformal field theory. JHEP 1604091.

179. Liendo P., Rastelli L., and van Rees B.C. 2013. The Bootstrap Program for Boundary CFT . JHEP 1307113.

180. Gadde A. 2016. Conformal constraints on defects. e-Print: arXiv:1602.06354.

181. Fukuda M., Kobayashi N. and Nishioka T. 2017. Operator product expansion for conformal defects. e-Print: arXiv:1710.11165.

182. Isachenkov M. and Schomerus V. 2016. Superintegrability of $d$-dimensional Conformal Blocks. Phys. Rev. Lett. 117 no. 7, 071602.

183. Calogero F. 1971. Solution of the one-dimensional $\mathrm{N}$ body problems with quadratic and/or inversely quadratic pair potentials. J. Math. Phys. 12419.

184. Sutherland B. 1972. Exact results for a quantum many body problem in one-dimension. 2. Phys. Rev. A5 1372.

185. Heckman GJ., and Opdam E.M. 1987. Root systems and hypergeometric functions. I. Compositio Mathematica 643

186. Isachenkov M. and Schomerus V. 2017. Integrability of Conformal Blocks I: CalogeroSutherland Scattering Theory. e-Print: arXiv:1711.06609.

187. Schomerus V., Sobko E. and Isachenkov M. 2017. Harmony of Spinning Conformal Blocks. JHEP 1703085.

188. Schomerus V. and Sobko E. 2017. From Spinning Conformal Blocks to Matrix CalogeroSutherland Models. e-Print: arXiv:1711.02022.

189. Olshanetsky M.A. and Perelomov A.M. 1983. Quantum Integrable Systems Related to Lie Algebras. Phys. Rept. 94313.

190. Feher L. and Pusztai B.G. 2010. Derivations of the trigonometric BC(n) Sutherland model by quantum Hamiltonian reduction. Rev. Math. Phys. 22699.

191. Isachenkov M., Liendo P., Linke Y., and Schomerus V., Calogero-Sutherland Approach to Defect Blocks. arXiv:1806.09703 [hep-th].

192. Nakayama Y. 2016. Bootstrapping critical Ising model on three-dimensional real projective space. Phys. Rev. Lett. 11614.

193. Hasegawa C. and Nakayama Y. 2017. $\epsilon$-Expansion in Critical $\phi^{3}$-Theory on Real Projective Space from Conformal Field Theory. Mod. Phys. Lett. A32 07.

194. Schweigert C., Fuchs J. and Runkel I. 2006. Categorification and correlation functions in conformal field theory. In: M. Sanz-Solé (ed.) et al.: International Congress of Mathematicians 2006. e-Print: arXiv:math.CT/0602079.

195. Kapustin A. and Saulina N. 2011. Surface operators in 3d Topological Field Theory and 2d Rational Conformal Field Theory. In: H. Sati (ed.) et al.: Mathematical Foundations of Quantum Field Theory and Perturbative String Theory. e-Print: arXiv:1012.0911. 
196. Fuchs J., Schweigert C. and Valentino A. 2013. Bicategories for boundary conditions and for surface defects in 3-d TFT. Comm. Math. Phys. 321.

197. Lyubashenko VV. 1995. Invariants of three manifolds and projective representations of mapping class groups via quantum groups at roots of unity. Comm. Math. Phys. 172.

198. Bakalov B. and Kirillov A.N. 2000. On the Lego-Teichmüller game. Transform. Groups 5.

199. Fuchs J. and Schweigert C. 2017. Consistent systems of correlators in non-semisimple conformal field theory. Adv. Math. 307.

200. Fuchs J., Schweigert C. and Stigner C. 2012.Modular invariant Frobenius algebras from ribbon Hopf algebra automorphisms. J. Algebra 363.

201. Fuchs J., Schweigert C. and Stigner C. 2012. The Cardy-Cartan modular invariant. In: A. Rebhan (ed.) et al.: Strings, Gauge Fields, and the Geometry Behind. e-Print: arXiv:1201.4267 [hep-th].

202. Fuchs J., Gannon T., Schaumann G., and Schweigert C. 2018. The logarithmic Cardy case: Boundary states and annuli. Nucl. Phys. B 930287

203. Friedan, D. 2016. Quantum field theories of extended objects. e-Print: arXiv:1605.03279 [hepth].

204. Friedan, D. 2017. A new kind of quantum field theory of (n-1)-dimensional defects in $2 n$ dimensions. e-Print: arXiv:1711.05049 [hep-th].

205. http://www.physics.rutgers.edu/ friedan/\#res

206. Federer H, and Fleming W.H. 1960. Normal and integral currents. Ann. of Math. 272 458-520.

207. Ambrosio L., and Kirchheim B. 2000. Currents in metric spaces. Acta Math. 185 1, 1-80.

208. Friedan D. and Konechny A. 2004. On the boundary entropy of one-dimensional quantum systems at low temperature. Phys. Rev. Lett. 93030402

209. Casini H, Landea I.S., and Torroba G. 2016 The g-theorem and quantum information theory. JHEP 1610140

210. Bianchi L., Lemos M., and Meineri M. Line defects and radiation in $\mathcal{N}=2$ theories. arXiv:1805.04111 [hep-th]. 